\title{
THE ORIGIN OF [O II] IN POST-STARBURST AND RED-SEQUENCE GALAXIES IN HIGH-REDSHIFT CLUSTERS
}

\author{
B. C. Lemaux, L. M. Lubin, A. Shapley ${ }^{1}$, D. Kocevski, R. R. Gal ${ }^{2}$, and G. K. Squires ${ }^{3}$ \\ Department of Physics, University of California, Davis, 1 Shields Avenue, Davis, CA 95616, USA; lemaux @ physics.ucdavis.edu \\ Received 2009 October 11; accepted 2010 April 27; published 2010 May 25
}

\begin{abstract}
We present the first results from a near-IR spectroscopic campaign of the Cl1604 supercluster at $z \sim 0.9$ and the cluster RX J1821.6+6827 at $z \sim 0.82$ to investigate the nature of [O $\mathrm{II}] \lambda 3727$ emission in cluster galaxies at high redshift. Of the 401 members in Cl1604 and RX J1821+6827 confirmed using the Keck II/DEIMOS spectrograph, 131 galaxies have detectable [O II] emission with no other signs of current star formation activity, as well as strong absorption features indicative of a well-established older stellar population. The combination of these features suggests that the primary source of [O II] emission in these galaxies is not a result of star formation processes, but rather due to the presence of a low-ionization nuclear emission-line region (LINER) or Seyfert component. Using the NIRSPEC spectrograph on the Keck II $10 \mathrm{~m}$ telescope, 19 such galaxies were targeted, as well as 6 additional [O II]-emitting cluster members that exhibited signs of ongoing star formation activity. Nearly half $(\sim 47 \%)$ of the 19 [O II]-emitting, absorption-line-dominated galaxies exhibit [O II] to $\mathrm{H} \alpha$ equivalent width (EW) ratios higher than unity, the typical observed value for star-forming galaxies, with an EW distribution similar to that observed for LINERs at low redshift. A majority ( $68 \%)$ of these 19 galaxies are classified as LINER/Seyfert based primarily on the emission-line ratio of [N II] $\lambda 6584$ and $\mathrm{H} \alpha$. The fraction of LINER/Seyferts increases to $\sim 85 \%$ for red [O II]emitting, absorption-line-dominated galaxies. The LINER/Seyfert galaxies in our Cl1604 sample exhibit average $L([\mathrm{O} \mathrm{II}]) / L(\mathrm{H} \alpha)$ ratios that are significantly higher than that observed in populations of star-forming galaxies, suggesting that $[\mathrm{O} \mathrm{II}]$ is a poor indicator of star formation in a significant fraction of high-redshift cluster members. From the prevalence of [O II]-emitting, absorption-line-dominated galaxies in both systems and the fraction of such galaxies that are classified as LINER/Seyfert, we estimate that at least $\sim 20 \%$ of galaxies in high-redshift clusters with $M_{\star}>10^{10}-10^{10.5} M_{\odot}$ contain a LINER/Seyfert component that can be revealed with line ratios. We also investigate the effect such a population has on the global star formation rate of cluster galaxies and the post-starburst fraction, concluding that LINER/Seyferts must be accounted for if these quantities are to be physically meaningful.
\end{abstract}

Key words: galaxies: active - galaxies: clusters: general - galaxies: evolution - galaxies: formation - infrared: general - techniques: spectroscopic

Online-only material: color figures

\section{INTRODUCTION}

At low redshift, the final result of galaxy processing and gas depletion in cluster galaxies is widely observed. Most galaxy populations in low-redshift clusters are dominated by bright early-type galaxies, primarily devoid of star formation (Dressler et al. 1985, 2004; Balogh et al. 1997; Hashimoto et al. 1998; Lewis et al. 2002; Gómez et al. 2003; Pimbblet et al. 2006). At higher redshifts $(z \sim 0.4-1)$ where this processing has had less time to occur, the fraction of late-type and active or recently star-forming galaxies increases (Dressler \& Gunn 1988; Couch et al. 1994; Dressler et al. 1997, 2004, 2009; van Dokkum et al. 2000; Lubin et al. 2002; Poggianti et al. 2006; Oemler et al. 2009). However, the physical processes that are responsible for the quenching of star formation and the transformation of disk galaxies to dormant spheroids over the last $\sim 7 \mathrm{Gyr}$ are still not well understood.

To accurately quantify this evolution, it is essential to use diagnostics that are valid and accessible across a broad redshift range. To determine the rate at which a galaxy is forming stars,

\footnotetext{
1 Also at Department of Physics and Astronomy, 430 Portola Plaza, University of California, Los Angeles, CA 90095-1547, USA.

2 Also at University of Hawai' i, Institute for Astronomy, 2680 Woodlawn Drive, Honolulu, HI 96822, USA.

3 Also at California Institute of Technology, M/S 220-6, 1200 E. California Blvd., Pasadena, CA 91125, USA.
}

the $\mathrm{H} \alpha$ line at $6563 \AA$ is typically used, as it is a relatively dust-independent measure of the star formation rate (SFR) in the last $10 \mathrm{Myr}$. As $\mathrm{H} \alpha$ moves out of the optical window other spectral lines must be used to determine galaxy SFRs. Many higher redshift surveys $(0.6 \leqslant z \leqslant 1.4)$ use instead the [O II] doublet at $3727 \AA$ as a proxy for $\mathrm{H} \alpha$ since it is traditionally associated with nebular regions of current star formation and is less sensitive to stellar absorption than higher order Balmer lines (e.g., Cooper et al. 2006; Vergani et al. 2008).

However, a comprehensive study by Yan et al. (2006, hereafter Y06) of 55,000 Sloan Digital Sky Survey (SDSS) galaxies at low redshift $(0.07 \leqslant z \leqslant 0.1)$ suggests that [O II] emission is a poor indicator of the SFR in many galaxies. While a large fraction of blue star-forming galaxies in the sample have appreciable [O II] emission, approximately $40 \%$ of the red, early-type galaxies also show moderate to strong [O II] emission. In $91 \%$ of the latter the $[\mathrm{O} \mathrm{II}]$ emission likely does not originate from normal star formation processes. Rather, the strengths of [N II] $\lambda 6584$ relative to $\mathrm{H} \alpha$ and [O III] $\lambda 5007$ relative to $\mathrm{H} \beta \lambda 4861$ (i.e., a BPT diagram; Baldwin et al. 1981) for those [O II]-emitting redsequence galaxies with all five features detected indicate that the line emission is related either to active galactic nuclei (AGNs) or other galactic processes not associated with star formation. Most commonly, the source of [O II] emission in these galaxies is related to processes associated with low-ionization nuclear emission-line regions (LINERs). 
This result has significant consequences for galaxy evolution studies. For galaxy populations that are currently forming stars in addition to having LINER activity, using [O II] as an SFR indicator results in an overestimate of the global SFR. More importantly, since $[\mathrm{O} I \mathrm{II}$ probes both the star formation and LINER activity, accurately identifying galaxies in a transitory phase following the truncation of a star formation event (i.e., "K+A" or "E+A" galaxies; Dressler \& Gunn 1983, 1992) becomes more difficult when [O II] is used as the sole SFR indicator. Although rare $(\lesssim 2 \%)$ among bright galaxies both in nearby clusters and in the local and distant field populations (Zabludoff et al. 1996; Dressler et al. 1999; Goto et al. 2003; Tran et al. 2003; Quintero et al. 2004; Yan et al. 2009), K+A galaxies typically comprise a significant fraction $(15 \%-25 \%)$ of the galaxy populations in distant clusters (Dressler et al. 1999, 2004; Tran et al. 2003; Oemler et al. 2009). The correct classification of such galaxies is a vital step in linking the large number of star-forming, disk galaxies seen in high-redshift clusters to the quiescent, early-type galaxies observed in their local counterparts (e.g., Poggianti et al. 1999; Oemler et al. 2009; Wild et al. 2009).

The $\mathrm{K}+\mathrm{A}$ classification is based on two physical properties of galaxies: significant recent star formation and the absence of current star formation. The second criterion as applied to highredshift galaxies $(z \gtrsim 0.3)$ typically requires that the galaxy spectra be essentially devoid of [O II] emission (Dressler \& Gunn 1992; Zabludoff et al. 1996; Balogh et al. 1999; Dressler et al. 2004; Oemler et al. 2009). If this phase of LINER emission is a typical stage of galaxy evolution, excluding all [O II] emitters from $\mathrm{K}+\mathrm{A}$ samples severely underestimates the fraction of galaxies that are truly "post-starburst" or "post-starforming." In the low-redshift sample of Y06 a large fraction $(\sim 80 \%)$ of the $\mathrm{K}+\mathrm{A}$ population (selected to exclude current star formation on the basis of the $\mathrm{H} \alpha$ line) show appreciable levels of [O II] emission. If cluster galaxies at high redshift share similar properties, it is necessary to understand where galaxies that exhibit LINER emission lie along the evolutionary chain in clusters and what role, if any, LINER emission has in truncating star formation in cluster galaxies.

To study the properties of this phenomenon at high redshift, we use the extensive spectroscopic database from the Observations of Redshift Evolution in Large Scale Environments (ORELSE) survey (Lubin et al. 2009, hereafter L09). The ORELSE survey is an ongoing multi-wavelength campaign mapping out the environmental effects on galaxy evolution in the large scale structures surrounding 20 known clusters at moderate redshift $(0.6 \leqslant z \leqslant 1.3)$. In particular, this paper focuses on two structures, the Cl1604 supercluster at $z \approx 0.9$ and the $X$-ray-selected cluster RX J1821.6+6827 at $z \approx 0.82$. Combining the wealth of previous ORELSE observations in these fields with newly obtained Keck II Near-Infrared Echelle Spectrograph (NIRSPEC) spectroscopy of 25 galaxies, we investigate the pervasiveness of LINER emission in cluster galaxies at high redshift and the properties of galaxies whose optical emission lines are dominated by this phenomenon.

The remainder of the paper is organized as follows: in Section 2, we discuss the two high-redshift structures targeted by this survey. Section 3 describes the optical spectroscopy and discusses the target selection, observation, and reduction of the near-infrared spectroscopy. In Section 4, we describe our methods for equivalent width (EW), relative flux, and absolute flux measurements, including absolute spectrophotometric calibration and extinction corrections. In Section 5, we present our results and discuss their consequences for high-redshift galaxy surveys. Section 6 presents our conclusions. We adopt a standard concordance $\Lambda$ CDM cosmology with $H_{0}=70 \mathrm{~km} \mathrm{~s}^{-1}$, $\Omega_{\Lambda}=0.7$, and $\Omega_{M}=0.3$. All EW measurements are presented in the rest frame and all magnitudes are given in the $\mathrm{AB}$ system (Oke \& Gunn 1983; Fukugita et al. 1996).

\section{TARGETED STRUCTURES}

To quantify the frequency of LINER emission in cluster galaxies at high redshift, we study the galaxy population in the optically selected $\mathrm{Cl} 1604$ supercluster at $z \approx 0.9$. The supercluster is a massive collection of eight or more constituent groups and clusters, spanning $13 \mathrm{~h}^{-1}$ comoving Mpc in the transverse dimensions and nearly $100 \mathrm{~h}^{-1}$ comoving Mpc in the radial dimension (see Gal et al. 2008 for details, hereafter G08). Additionally, we target galaxies in the X-ray-selected cluster RX J1821.6+6827 (i.e., NEP5281) at $z \approx 0.82$. The properties and data available for each structure are discussed individually below.

\subsection{The Cl1604 Supercluster}

The $\mathrm{Cl} 1604$ supercluster consists of structures that range from rich, virialized clusters dominated by red, early-type galaxies and a hot intracluster medium (clusters Cl1604+4304 and Cl1604+4314, hereafter clusters A and B) to sparse chains of galaxies dominated by starbursts and luminous AGN (e.g., Kocevski et al. 2009a, 2009b). The velocity dispersions of the structures in Cl1604 range from $811 \pm 76 \mathrm{~km} \mathrm{~s}^{-1}$ (cluster B)

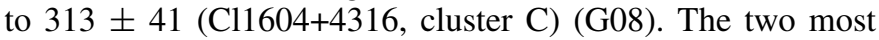
massive clusters (clusters $\mathrm{A}$ and $\mathrm{B}$ ) have well-measured bolometric X-ray luminosities $\left(L_{X, \mathrm{Bol}}=15.76 \pm 1.48\right.$ and $11.64 \pm$ $1.49 \times 10^{43} h_{70}^{-1} \mathrm{erg} \mathrm{s}^{-1}$, respectively) and X-ray temperatures $\left(T_{X}=3.50_{-1.08}^{+1.82}\right.$ and $\left.1.64_{-0.45}^{+0.65} \mathrm{keV}\right)$, while the other groups and clusters show no evidence of a hot intracluster medium ( $L_{X, \text { bol }} \lesssim 7.4 \times 10^{43} h_{70}^{-1} \mathrm{erg} \mathrm{s}^{-1}$; Kocevski et al. 2009a).

The imaging data on this structure include Very Large Array (VLA; B-array, $20 \mathrm{~cm})$, Spitzer IRAC (3.6/4.5/5.8/8.0 $\mu \mathrm{m})$ and MIPS $24 \mu \mathrm{m}$ imaging, archival $V$-band Suprimecam imaging, deep Palomar $5 \mathrm{~m} r^{\prime} i^{\prime} z^{\prime} K_{s}$ imaging, 17 Hubble Space Telescope (HST) Advanced Camera for Surveys (ACS) pointings in $F 606 \mathrm{~W}$ and $F 814 W$, and two deep (50 ks) Chandra pointings. The reduction of some of the multi-wavelength imaging on the supercluster has been discussed in other papers (Kocevski et al. 2009a, 2009b; D. D. Kocevski et al. 2010, in preparation). The analysis of these ancillary data as it pertains to the current sample will be discussed in a second paper that focuses on the multiwavelength and stellar mass properties of galaxies studied in this paper (B. C. Lemaux et al. 2010, in preparation).

\section{2. $R X J 1821.6+6827$}

The cluster RX J1821.6+6827 (hereafter RX J1821) at $z \approx$ 0.82 was originally observed by ROSAT (Trümper 1982) in the North Ecliptic Pole Survey (Henry et al. 2001; Mullis 2001; Gioia et al. 2003). Spectroscopic observations as part of the ORELSE survey (L09) yielded a velocity dispersion of $926 \pm$ $77 \mathrm{~km} \mathrm{~s}^{-1}$ from 40 cluster members within $1 h^{-1} \mathrm{Mpc}$ of the cluster center, slightly higher than the most massive cluster in Cl1604. The X-ray temperature and bolometric luminosity of the cluster $\left(4.7_{-0.7}^{+1.3} \mathrm{keV}\right.$ and $1.17_{-0.18}^{+0.13} \times 10^{45} h_{70}^{-2} \mathrm{erg} \mathrm{s}^{-1}$, 
respectively) derived from $X M M-N e w t o n$ observations suggest that the cluster is reasonably relaxed, as it lies close to the $\sigma_{v}-T$ relationship observed in virialized clusters. However, the X-ray morphology is elongated (Gioia et al. 2004), and measurable velocity substructure has been identified in the spectroscopy (see L09), implying that the cluster is still in the process of formation. Still, RX J1821 represents a higher mass, higher temperature cluster than those in $\mathrm{Cl1604}$, allowing us to measure the pervasiveness of LINER activity in high-redshift clusters at significantly different stages in their dynamical evolution.

The wealth of imaging available for this structure is similar to that for Cl1604, including VLA (B-array, $20 \mathrm{~cm}$ ), Spitzer IRAC (3.6/4.5/5.8/8.0 $\mu \mathrm{m})$ and MIPS 24/70 $\mu \mathrm{m}$ imaging, deep Palomar $5 \mathrm{~m} r^{\prime} i^{\prime} z^{\prime}$ and Kitt Peak $4 \mathrm{~m} K_{s}$ imaging, and a single deep (50 ks) Chandra pointing.

\section{OBSERVATIONS}

\subsection{Optical Spectroscopy}

\subsubsection{Low-Resolution Imaging Spectrometer}

The original spectroscopic campaign in Cl1604 was conducted with LRIS on the Keck $10 \mathrm{~m}$ telescopes, obtaining spectra of all galaxies with $R<23$ in the vicinity of clusters C11604+4304 and C11604+4321. Further details on the galaxy selection process, observations, and reduction of these data are given in the original survey paper of Oke et al. (1998).

Following the original survey, a follow-up LRIS spectroscopic campaign consisting of six slitmasks was undertaken in the $\mathrm{C} 11604$ field and is described in detail in Gal \& Lubin (2004). Since only one of the LRIS spectra from these observations is used in this study, we only briefly give the details of the LRIS observations. The LRIS spectroscopic targets in the follow-up campaign were observed with the $4001 \mathrm{~mm}^{-1}$ grating in multi-object slitmask mode, with an FWHM resolution of $\sim 7.8 \AA$ and a typical wavelength coverage of 5000-9000. In total, 85 high-quality redshifts were obtained with LRIS with $0.84 \leqslant z \leqslant 0.96$, the adopted redshift range of the Cl1604 supercluster. All galaxies in RX J1821 that are used for this study were observed with DEep-Imaging Multi-Object Spectrograph (DEIMOS); thus, we do not include any additional information on the original LRIS campaign of Gioia et al. (2004) for this system.

\subsubsection{DEep-Imaging Multi-Object Spectrometer}

The bulk of the redshifts in the $\mathrm{Cl1604}$ field come from observations of 12 slitmasks with the DEIMOS (Faber et al. 2003) on the Keck II 10 m telescope between 2003 May and 2007 June. The details of the observations and spectroscopic selection are described in G08. Briefly, slitmasks were observed with the $1200 \mathrm{l} \mathrm{mm}^{-1}$ grating with an FWHM resolution of $\sim 1.7 \AA\left(68 \mathrm{~km} \mathrm{~s}^{-1}\right)$ and a typical wavelength coverage of 6385-9015 $\AA$. The spectroscopic targets for these slits were selected based on the likelihood of being a cluster member, determined through a series of color and magnitude selections based on data obtained from the Palomar $5 \mathrm{~m}$ Large Format Camera (LFC; Simcoe et al. 2000). The slitmasks were observed with differing total integration times depending on weather and seeing conditions. Integration times varied from $7200 \mathrm{~s}$ to $14,400 \mathrm{~s}$ in seeing that ranged from 0.52 to $1^{\prime \prime} .4$.

In total, 903 total high-quality $(Q \geqslant 3$; see G08 for detailed explanations of the quality codes) extragalactic DEIMOS spectra were obtained in the Cl1604 field, with 329 within the adopted redshift range of the supercluster. Combining these results with the LRIS campaigns, 414 high-quality spectra have been obtained for members of the Cl1604 supercluster. The spectroscopic survey is not explicitly magnitude limited; however, based on the turnover in the number counts of galaxies with high-quality spectra, we estimate that our spectroscopic sample is representative of all galaxies brighter than $i^{\prime} \sim 23$ (F814W $\sim 22.5$ ). The limiting magnitude of the spectral sample is much fainter than the turnover magnitude, probing galaxies down to $i^{\prime}=25.2(F 814 W \sim 26)$.

Two DEIMOS slitmasks covering the RX J1821 field were observed in 2005 September. The spectroscopic targets for these slits were selected in a nearly identical way to targets in Cl1604. The selection process differed only in the LFC color-magnitude cuts that were introduced to account for the small difference in rest-frame bandpasses of our filters at the redshifts of the two structures (see L09 for more details). Both slitmasks were observed for $5 \times 1800 \mathrm{~s}$ under photometric conditions, with typical seeing of $00^{\prime \prime} 6-00^{\prime} 8$. The spectroscopic setup (grating used, blocking filter, slit widths, and central wavelength) was identical to that of the C11604 DEIMOS observations.

Of the 20 original cluster members observed by Gioia et al. (2004) in RX J1821, 7 were re-observed with DEIMOS. In total, 189 high-quality $(Q \geqslant 3)$ redshifts were obtained from DEIMOS observations of the RX J1821 field, with 73 galaxies lying between $0.805 \leqslant z \leqslant 0.83$. With the 12 additional redshifts obtained by Gioia et al. (2004) within the adopted redshift range, the total spectroscopic database for RX J1821 contains 85 cluster members. Similar to $\mathrm{Cl1604}$, the spectroscopic data in this system is also representative for galaxies brighter than $i^{\prime} \sim 23$, with a limiting magnitude of $i^{\prime}=24$.

The exposure frames for each slitmask in the C11604 and RX J1821 fields were combined using the DEEP2 version of the spec $2 d$ package (Davis et al. 2003). This package combines the individual science exposures of the slitmask and performs wavelength calibration, cosmic ray removal, and sky subtraction on a slit-by-slit basis, generating processed two-dimensional and one-dimensional spectra for each slit. Further details of the spec $2 d$ package and the reduction process are given in Lemaux et al. (2009, hereafter Lem09).

Not surprisingly, the DEIMOS spectral properties of the galaxy population in RX J1821 differ appreciably from those in Cl1604. While $63 \%$ of galaxies observed with DEIMOS in the $\mathrm{Cl1604}$ supercluster have detectable [O II] $\lambda 3727$ (hereafter [O II]) emission, a clear sign of either AGN or star-forming activity, only $36 \%$ of galaxies observed with DEIMOS in RX J1821 show similar activity, typically at lower levels. The galaxy population in $\mathrm{Cl1604}$ also seems to have been more

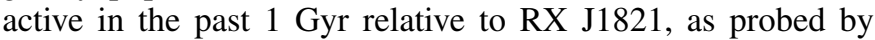
the average strength of the $\mathrm{H} \delta$ absorption line. The spectra of the C11604 supercluster members contain Balmer absorption strengths typical of galaxies with significant star formation in the recent past, whereas the spectra of RX J1821 members are, on average, typical of galaxies with no active star formation in the last 1 Gyr.

While RX J1821 is more sparsely sampled than C11604, we have sub-sampled the C11604 DEIMOS spectroscopic data so that it is equivalent to that of RX J1821. A significant difference in the fraction of [O II] emitters and the strength of the Balmer absorption features between the two fields is still present. This result suggests that the variance in mean spectroscopic properties between the two structures reflects true differences in the galaxy populations. While we select galaxies with similar 
DEIMOS/LRIS spectra in Cl1604 and RX J1821 for nearinfrared spectroscopy (see Section 3.2.1), the environments of these galaxies are significantly different. If LINER-type processes in galaxies are only induced by very specific processes (i.e., ram-pressure stripping, harassment, galaxy merging, etc.) or are limited to very specific stages in a galaxy's evolution, there should also be clear differences in the observed-frame near-infrared emission-line properties of the galaxies in the two structures.

\subsection{Near-Infrared Spectroscopy}

\subsubsection{NIRSPEC Target Selection}

Twenty-five galaxies were targeted for follow-up observations in the two structures with the NIRSPEC spectrograph (McLean et al. 1998) on the Keck II $10 \mathrm{~m}$ telescope. Since this sample consists of a small fraction $(\sim 5 \%)$ of the galaxies in Cl1604 and RX J1821, the philosophy adopted in the target selection was to maximize our chances at successfully detecting LINER-type galaxies with our observations.

Our highest priority sample (priority 1) are galaxies with spectra that would be classified as either quiescent $(\mathrm{K})$ or poststarburst $(\mathrm{K}+\mathrm{A})$ based on the bulk of the spectral features, but which also show low to moderately high levels of [O II] emission $(2 \AA<\mathrm{EW}([\mathrm{O} \mathrm{II}])<74 \AA$, where a positive EW corresponds to a feature in emission, see Section 4.1 and Appendix A). The priority 1 sample consists of 109 galaxies in C11604 and 23 galaxies in RX J1821, of which 19 were observed with NIRSPEC (17 in Cl1604 and 2 in RX J1821). While 52\% of the galaxies in the $\mathrm{Cl1604}$ priority 1 sample would be classified as post-starburst based on the strength of $\mathrm{H} \delta$ alone (i.e., $\mathrm{EW}(\mathrm{H} \delta)<-5 \AA$ ), we did not impose an $\mathrm{H} \delta$ cut for priority 1 targets. Priority 1 galaxies that were observed with NIRSPEC were primarily selected to maximize the total number of priority 1 galaxies in our sample. Thus, priority 1 galaxies with a close $\left(r<24^{\prime \prime}\right)$ priority 1 companion were favored. The consequences of this choice, as well any possible bias that is introduced as a result, are discussed later in this section.

All priority 1 galaxies have strong $\mathrm{Ca} \mathrm{H}$ and $\mathrm{K}$ lines, and a majority have Balmer absorption features, suggesting that star formation has been suppressed within at least the last $\sim 1 \mathrm{Gyr}$. While [O II] emission usually precludes the classification of a galaxy as post-starburst or quiescent (e.g., Balogh et al. 1999; Dressler et al. 1999; Oemler et al. 2009; though not always, see Wild et al. 2009, and references therein), the spectra of these galaxies strongly suggest a different interpretation. Strong $\mathrm{Ca} \mathrm{H}$ and $\mathrm{K}$ features indicate the presence of a well-established older stellar population and are typically absent following a significant star formation event when the continuum light is dominated by $\mathrm{O}$ and B stars. Similarly, emission from H II regions can mask Balmer absorption features even in the case of relatively minor star formation events (e.g., Taniguchi et al. 2000), although the strength of the star formation event needed is somewhat sensitive to the star formation history of the galaxy. The presence of these features in priority 1 galaxies suggests that they are not undergoing star formation episodes and the [O II] emission does not originate from $\mathrm{H}$ II regions.

The remaining spectral classes (priorities 2-4) are used to place a second target on our slit. Priority 2 (91 galaxies in C11604 and 5 galaxies in RX J1821) are galaxies that exhibit either [O II] in emission with no other strong spectral features or [O II] in emission plus strong $\mathrm{Ca} \mathrm{H}$ and $\mathrm{K}$, but with other signs of ongoing star formation (i.e., at least one of the higher order Balmer lines was observed in emission). Galaxies with no $\mathrm{Ca} \mathrm{H}$ and $\mathrm{K}$ and obvious signs of ongoing star formation and galaxies that have no features in emission were given the lowest priorities (priorities 3 and 4 with 52 and 62 galaxies, respectively, in Cl1604; 2 and 42 galaxies, respectively, in RX J1821). Six galaxies of lower priorities were observed with NIRSPEC, four in Cl1604 and two in RX J1821.

The remaining 16 galaxies observed with DEIMOS in $\mathrm{Cl} 1604$ could not be classified due to reduction artifacts that prevented us from accurately measuring the strength of spectral features. Only one galaxy originally targeted by LRIS was chosen as an NIRSPEC target due to the lower spectral resolution and lack of flux calibration in these data. The one LRIS target was chosen for NIRSPEC because it is the brightest red-sequence galaxy in $\mathrm{Cl1604}\left(z^{\prime}=19.42, F 814 W=20.84\right)$, is a strong radio emitter, and has a priority 1 spectrum. The appreciable [O II] emission $(\mathrm{EW}([\mathrm{O} \mathrm{II}])=6.3 \AA)$ present in the spectrum of this galaxy was a sufficient mystery for us to warrant targeting.

Initially, the selection process for NIRSPEC targets only involved the use of the DEIMOS spectral data. Following these initial observations, we included broadband color cuts, which was used to differentiate between galaxies on the observed ACS red sequence and those blueward of it. As nearly all of the priority 1 galaxies in RX J1821 lie on the observed LFC red sequence (see Section 5.3), we did not apply any broadband color selection to the potential NIRSPEC targets in this field. These color criteria were imposed in $\mathrm{Cl1604}$ in order to favor [O II]-emitting red-sequence galaxies, which were preferred as targets as a large fraction (91\%) of such galaxies at low-redshift exhibit [O II] emission that is inconsistent or likely inconsistent with normal star-forming processes (Y06). If galaxies at higher redshift exhibit similar trends, selecting red-sequence galaxies greatly improves our chances of successfully observing galaxies that contain a LINER component.

To demonstrate the differences between the various priority classes used for NIRSPEC observations, we use the full C11604 DEIMOS spectroscopy. Figure 1 shows the spectral "co-addition" of the galaxies that comprise each priority class. The co-additions were performed in a manner nearly identical to that of Lem09. In order to compensate for the effects of slit-loss, the flux in each DEIMOS spectrum was normalized to unity and re-weighted by the absolute $i^{\prime}$ magnitude of the galaxy. The differences between the composite properties of the priority classes can be seen clearly in the spectra.

We also required that galaxies have $z \leqslant 0.93$, so that any observed $\mathrm{H} \alpha$ emission is blueward of the strong $\mathrm{OH}$ airglow lines at $\lambda \approx 1.27 \mu \mathrm{m}$ (see Figure 2 ). Additionally, we required any potential target to have a nearby supercluster member that could also be placed on the slit during the observations. This constraint requires that our targets have at least one other spectroscopically confirmed supercluster member within $24^{\prime \prime}$. Because the targeted pairs are kinematically related at a rate similar to all galaxies with similar separations, this criterion adds no additional bias to our sample as we are sampling galaxies with clustering properties representative of the majority of supercluster members.

Figure 3 shows the color-magnitude diagram (CMD) of all four NIRSPEC priority classes in the $\mathrm{Cl1604}$ supercluster that have DEIMOS spectra and the one targeted LRIS object covered by our ACS pointings. The galaxies comprising the three lowest priorities each occupy different, fairly well-defined regions in the CMD. The lowest priority targets (priority 4, labeled "K or $\mathrm{K}+\mathrm{A}$ ") almost exclusively lie on the red sequence (see 


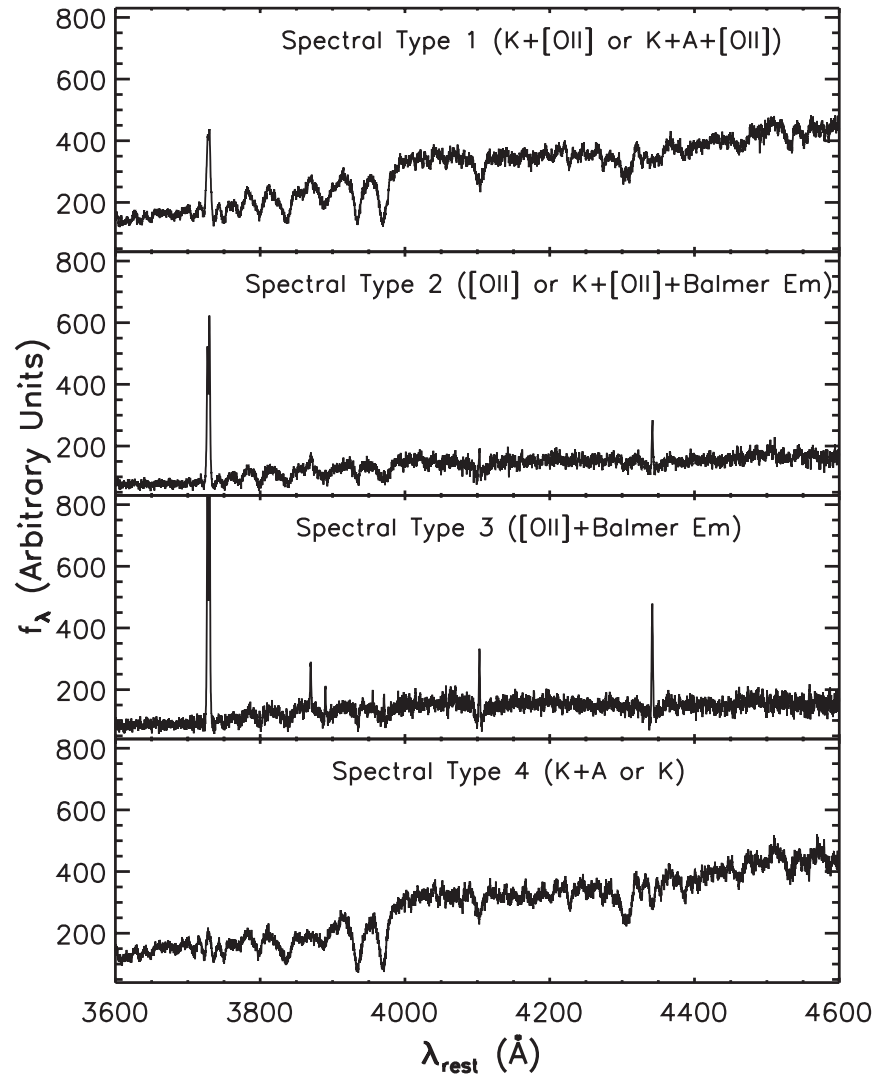

Figure 1. DEIMOS spectral co-additions of potential NIRSPEC targets in the $\mathrm{Cl1604}$ supercluster members. The co-additions of four priority classes selected primarily by the absence or presence of certain spectral features (see Section 3.2.1) are shown. Each co-addition is a luminosity inverse variance weighted mean. Priority 1 galaxies (top panel, 108 galaxies) exhibit strong $\mathrm{Ca}$ $\mathrm{H}$ and $\mathrm{K}$ and Balmer absorption features, as well as a moderately strong $4000 \AA$ break, indicative of a dominate older stellar population and recently truncated star formation. However, strong [O II] emission is also present suggesting that these systems may be still forming stars or, alternatively, have some contribution from a LINER or Seyfert. Priority 1 galaxies constituted 76\% (19/25) of the galaxies targeted with NIRSPEC. Priority 2, 3, and 4 populations, from which the six other targets were drawn, have co-additions performed with 91,52 , and 62 galaxies, respectively.

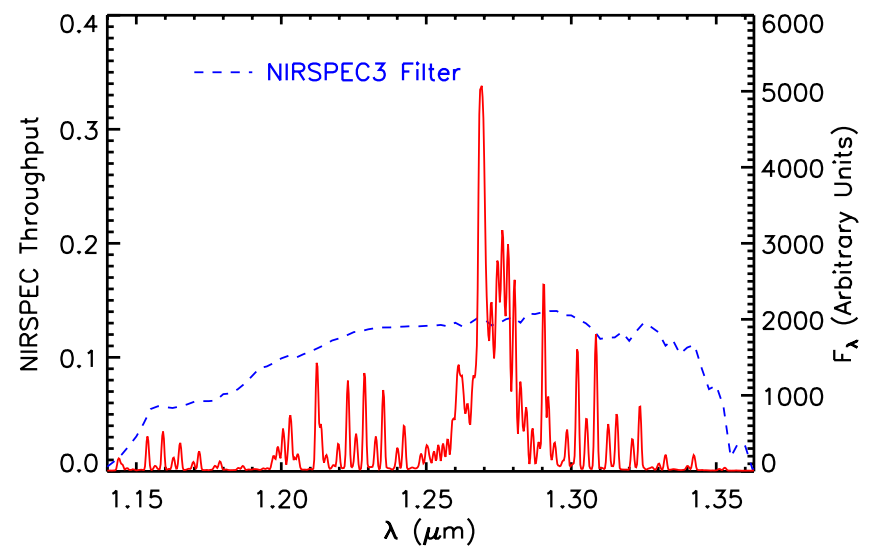

Figure 2. Throughput curve of the NIRSPEC-3 ( $J$-band) filter against the backdrop of the near-IR night sky airglow lines. The relative intensity of the $\mathrm{OH}$ skylines has been scaled for clarity. NIRSPEC throughput includes light lost from a point source due to the slit as well as losses associated with the telescope. All NIRSPEC targets have $z \leqslant 0.93$, which allowed observed-frame $\mathrm{H} \alpha$ and $[\mathrm{N} \mathrm{II}] \lambda 6584$ to avoid the strong $\mathrm{OH}$ features at $\lambda \approx 1.27 \mu \mathrm{m}$.

(A color version of this figure is available in the online journal.)

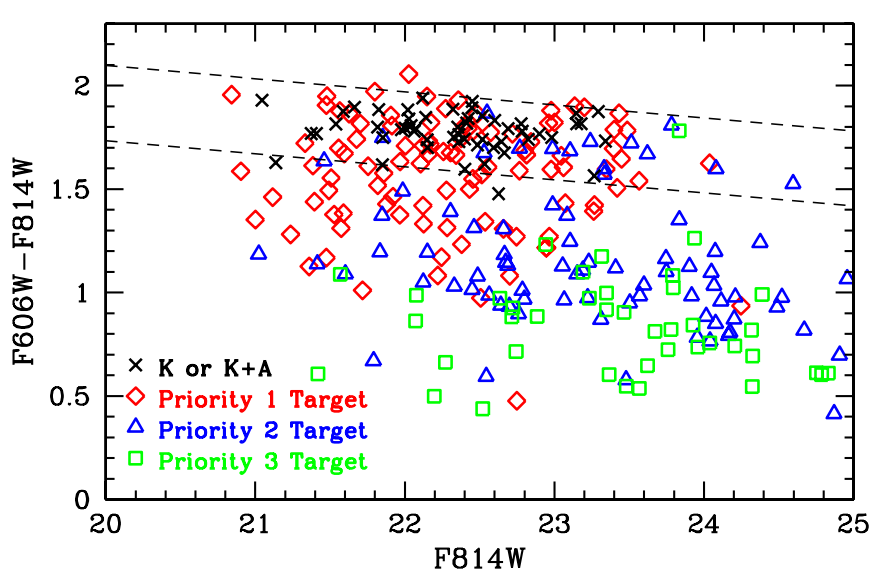

Figure 3. Color-magnitude diagram of the 279 DEIMOS confirmed Cl1604 members observed with ACS that were classifiable. Also included is the one LRIS confirmed member that was targeted by NIRSPEC. Galaxies with no ongoing star formation (priority 4) are confined almost exclusively to the $\mathrm{Cl1604}$ red sequence. Galaxies that are likely undergoing moderate (priority 2) or high (priority 3) levels of star formation activity are primarily found in the faint region of the blue cloud, though dusty starburst galaxies located on or near the red sequence are among the significant exceptions. Priority 1 galaxies, which represent the bulk of our NIRSPEC targets, cover a large dynamic range in color-magnitude space, comprising both the most luminous and least luminous red-sequence galaxies as well as a large portion of bright galaxies with bluer colors.

(A color version of this figure is available in the online journal.)

Section 5.3 for a detailed discussion on how the red sequence is defined). Priority 3 targets primarily occupy the bluest part of the "blue-cloud" region, and priority 2 targets primarily occupy the redder boundaries of the blue cloud. Our priority 1 targets are much more expansive, encompassing both the brightest and dimmest galaxies on the red sequence and the bulk of the bright galaxies blueward of the red sequence. The large extent of priority 1 galaxies in color-magnitude space suggests that, even though we are selecting galaxies with similar spectroscopic properties, we may be sampling galaxy populations at different stages in their evolution. Tables 1 and 2 list the details of each galaxy observed with NIRSPEC as well as their priority classes.

\subsubsection{NIRSPEC Observations}

In total, 25 galaxies were observed with NIRSPEC on the dates of 2007 June 4 and 2008 May 21 UTC, 21 in Cl1604 and 4 in RX J1821. The observations consisted of 19 priority 1 galaxies (17 in Cl1604 and 2 in RX J1821), hereafter referred to as our "main sample" and six priority 2-3 galaxies (4 in Cl1604 and 2 in RX J1821), hereafter referred to as our "filler sample." Observations for both nights were taken in low-resolution mode with slit widths of 0.76 , resulting in a pixel scale of $3 \AA$ pixel $^{-1}$ and an FWHM resolution of $\sim 8 \AA$. The observations were taken through the NIRSPEC-3 filter (similar to $J$ band; see Figure 2), with a typical wavelength coverage of $2900 \AA$ and central wavelength of $1.273 \mu \mathrm{m}$. Conditions on both nights were photometric and seeing ranged from $0.3-0.0^{\prime \prime} 4$ on the first night and $0.4-0$ '. 8 on the second night.

The NIRSPEC-3 filter was chosen to maximize the sensitivity of the instrument at $1.24 \mu \mathrm{m}$, roughly the wavelength of $\mathrm{H} \alpha$ and $[\mathrm{NII}]$ at the redshift of the $\mathrm{Cl1604}$ supercluster. While our spectral coverage included the [O I] $\lambda 6300$ feature (hereafter $\left[\mathrm{O}_{\mathrm{I}}\right]$ ), our integration times were only long enough to significantly detect [O I] in galaxies with extremely hard ionizing spectra. Our average detection significance of $\sim 7 \sigma$ in $F(\mathrm{H} \alpha)$ implies a $\sim 1.75 \sigma$ significance of the $\left[\mathrm{O}_{\mathrm{I}}\right]$ feature 
Table 1

Cl1604 NIRSPEC Observations

\begin{tabular}{|c|c|c|c|c|c|c|c|c|c|c|}
\hline ID & Galaxy Number & $\alpha_{2000}$ & $\delta_{2000}$ & $z$ & $F 606 \mathrm{~W}$ & $F 814 W$ & $F 606 W-F 814 W$ & Setup Number & $\tau_{\exp }$ & Class \\
\hline $\mathrm{J} 160344+432429$ & 0 & 240.9322226 & 43.4079759 & 0.9023 & 23.60 & 21.97 & 1.63 & 1 & $5 \times 900 \mathrm{~s}^{\mathrm{a}}$ & Priority 1 \\
\hline J160344+432428 & 1 & 240.9325941 & 43.4077202 & 0.9024 & 22.11 & 23.16 & 1.05 & 1 & $5 \times 900 \mathrm{~s}^{\mathrm{a}}$ & Priority 1 \\
\hline J160345+432419 & 2 & 240.9375426 & 43.4051985 & 0.8803 & 24.01 & 22.46 & 1.55 & 1 & $5 \times 900 \mathrm{~s}^{\mathrm{a}}$ & Priority 1 \\
\hline $\mathrm{J} 160342+432406$ & 3 & 240.9247136 & 43.4016956 & 0.8986 & 25.10 & 23.45 & 1.64 & 2 & $4 \times 900 \mathrm{~s}$ & Priority 1 \\
\hline $\mathrm{J} 160342+432403$ & 4 & 240.9250684 & 43.4006981 & 0.8959 & 24.12 & 22.38 & 1.74 & 2 & $4 \times 900 \mathrm{~s}$ & Priority 1 \\
\hline $\mathrm{J} 160330+432208$ & 5 & 240.8732075 & 43.3687725 & 0.8983 & 22.84 & 21.40 & 1.44 & 3 & $4 \times 900 \mathrm{~s}$ & Priority 1 \\
\hline $\mathrm{J} 160329+432204$ & 6 & 240.8697693 & 43.3676967 & 0.9045 & 23.54 & 22.12 & 1.42 & 3 & $4 \times 900 \mathrm{~s}$ & Priority 1 \\
\hline $\mathrm{J} 160416+431021$ & 7 & 241.0657080 & 43.1725670 & 0.8999 & 24.91 & 23.75 & 1.16 & 4 & $4 \times 900 \mathrm{~s}$ & Priority 3 (Filler) \\
\hline $\mathrm{J} 160416+431017$ & 8 & 241.0648269 & 43.1713681 & 0.8999 & 22.99 & 21.49 & 1.49 & 4 & $4 \times 900 \mathrm{~s}$ & Priority 1 \\
\hline $\mathrm{J} 160404+432445$ & 9 & 241.0150297 & 43.4124202 & 0.9017 & 23.60 & 22.72 & 0.88 & 5 & $4 \times 900 \mathrm{~s}$ & Priority 3 (filler) \\
\hline $\mathrm{J} 160403+432436$ & 10 & 241.0108301 & 43.4099384 & 0.9015 & 23.34 & 21.82 & 1.52 & 5 & $4 \times 900 \mathrm{~s}$ & Priority 1 \\
\hline $\mathrm{J} 160429+431956$ & 11 & 241.1195420 & 43.3321920 & 0.9185 & 24.48 & 23.76 & 0.72 & 6 & $2 \times 900 \mathrm{~s}$ & Priority 3 (filler) \\
\hline $\mathrm{J} 160428+431953$ & 12 & 241.1171400 & 43.3312750 & 0.9198 & 23.37 & 21.75 & 1.61 & 6 & $2 \times 900 \mathrm{~s}$ & Priority 1 \\
\hline $\mathrm{J} 160406+431542$ & 13 & 241.0276264 & 43.2615940 & 0.8674 & 23.44 & 21.57 & 1.88 & 8 & $4 \times 900 \mathrm{~s}$ & Priority $1+$ red sequence \\
\hline $\mathrm{J} 160407+431539$ & 14 & 241.0299022 & 43.2607188 & 0.8676 & 24.48 & 22.81 & 1.67 & 8 & $4 \times 900 \mathrm{~s}$ & Priority $1+$ red sequence \\
\hline $\mathrm{J} 160426+431423$ & 15 & 241.1100259 & 43.2397136 & 0.8676 & 23.43 & 21.48 & 1.95 & 9 & $3 \times 900 \mathrm{~s}$ & Priority $1+$ red sequence \\
\hline $\mathrm{J} 160426+431419$ & 16 & 241.1092254 & 43.2386527 & 0.8658 & 22.80 & 20.84 & 1.96 & 9 & $3 \times 900 \mathrm{~s}$ & Priority $1+$ red sequence \\
\hline $\mathrm{J} 160427+431501$ & 17 & 241.1104629 & 43.2503720 & 0.8601 & 23.77 & 21.80 & 1.97 & 10 & $4 \times 900 \mathrm{~s}$ & Priority $1+$ red sequence \\
\hline $\mathrm{J} 160426+431439$ & 18 & 241.1086670 & 43.2441610 & 0.8710 & 24.94 & 23.35 & 1.60 & 10 & $4 \times 900 \mathrm{~s}$ & Priority $1+$ red sequence \\
\hline $\mathrm{J} 160406+431825$ & 19 & 241.0243330 & 43.3068500 & 0.9189 & 25.59 & 23.78 & 1.81 & 11 & $5 \times 900 \mathrm{~s}^{\mathrm{a}}$ & Priority 2 (filler) \\
\hline $\mathrm{J} 160406+431809$ & 20 & 241.0266087 & 43.3024702 & 0.9195 & 24.29 & 22.36 & 1.93 & 11 & $5 \times 900 \mathrm{~s}^{\mathrm{a}}$ & Priority $1+$ red sequence \\
\hline
\end{tabular}

Note. ${ }^{a}$ One exposure was not usable due to guider issues.

Table 2

RX J1821 NIRSPEC Observations

\begin{tabular}{ccccccccccc}
\hline \hline ID & Galaxy Number & $\alpha_{2000}$ & $\delta_{2000}$ & $z$ & $i^{\prime}$ & $z^{\prime}$ & $i^{\prime}-z^{\prime}$ & Setup Number $^{*} \tau_{\exp }$ & Class $^{\mathrm{a}}$ \\
\hline $\mathrm{J} 182110+682350$ & 21 & 275.29224260 & 68.39710400 & 0.7960 & 21.41 & 20.76 & 0.65 & 7 & $4 \times 900 \mathrm{~s}$ & Priority 1 \\
$\mathrm{~J} 182108+682329$ & 22 & 275.28199650 & 68.39415620 & 0.8134 & 21.75 & 21.00 & 0.75 & 7 & $4 \times 900 \mathrm{~s}$ & Priority 1 \\
$\mathrm{J} 182121+682715$ & 23 & 275.33619440 & 68.45408210 & 0.8092 & 23.32 & 23.03 & 0.30 & 12 & $3 \times 900 \mathrm{~s}$ & Priority 2(filler) \\
$\mathrm{J} 182123+682714$ & 24 & 275.34600740 & 68.45392090 & 0.8093 & 23.72 & 23.36 & 0.36 & 12 & $3 \times 900 \mathrm{~s}$ & Priority 2(filler) \\
\hline
\end{tabular}

Note. ${ }^{a}$ Note that no color cut was imposed on any RX J1821 targets.

for typical LINER spectra, too weak to use as a meaningful diagnostic.

$\mathrm{H} \alpha$ and [N II] were chosen to discriminate between star formation and LINER or Seyfert emission. This distinction would not be possible if we had instead chosen to observe $\mathrm{H} \beta$ $\lambda 4861$ and [O III] $\lambda 5007$ (hereafter [O III]). Unfortunately, $\mathrm{H} \alpha$ and [N II] provide little power to discriminate between LINERs and Seyferts, which is typically done (when using spectral techniques) by the ratio of $\mathrm{H} \beta$ to [O III] or the ratio of [O II] to [O III]. While we attempt to separate the two populations using spectral diagnostics in this paper (see Section 5.2), our multiwavelength data will be useful in this regard and will be used to fully characterize the nature of such processes in a future paper.

Spectral setups typically consisted of two targets observed simultaneously. For each setup, we acquired targets by blind offsets from bright $\left(r^{\prime} \sim 17.5\right)$ stars. The observation of each setup consisted of staggering $900 \mathrm{~s}$ exposures between nods on the sky of 1".4-2".5 along the $42^{\prime \prime}$ slit. A different number of exposures were taken for each setup to achieve a similar emission-line signal-to-noise ratio $(\mathrm{S} / \mathrm{N})$, with the total integration times varying between 1800 and $3600 \mathrm{~s}$. Preference was given to setups in the Cl1604 supercluster, with galaxies in RX J1821 observed only when the Cl1604 field was unavailable. Two standard stars drawn from the UKIRT list of bright standards ${ }^{4}$ were observed on each night, HD105601 (A2) at evening twilight and HD203856 (A0) at morning twilight.

\footnotetext{
4 http://www.jach.hawaii.edu/UKIRT/astronomy/calib/phot_cal/ ukirt_stds.html
}

\subsubsection{NIRSPEC Data Reduction}

The NIRSPEC data were reduced using a combination of Image Reduction and Analysis Facility (IRAF; Tody 1993) scripts and a publicly available semi-automated Interactive Data Language (IDL) pipeline (G. Becker 2009, private communication). The IRAF scripts (described in detail in Erb et al. 2003) were used primarily to create stacked two-dimensional spectra for use as a visual guide throughout the reduction and analysis of the data. All results in this paper are presented from data reduced with the IDL pipeline.

The pipeline initially determines the position of an object on the slit as a function of position on the $1024 \times 1024$ NIRSPEC detector. A "slit-map," made by interpolating between observations of a standard star, is used to correct for variations of the spatial position of the science object along the $42^{\prime \prime}$ slit. During this process, an initial wavelength solution is also generated using the skylines in the observations of the standard stars.

Each science frame was processed by differencing the flatfielded, dark-subtracted science frame with a reference science frame. A second wavelength solution was performed by manually identifying several cleanly separated bright $J$-band airglow lines in the science frames. The resultant product is a cosmic ray cleaned, wavelength calibrated, dark-subtracted, flat-fielded two-dimensional spectrum. A one-dimensional spectrum is generated by collapsing the dispersion axis and fitting an optimal Gaussian to observed peaks in the brightness distribution. At each wavelength position, the flux values for the one-dimensional spectrum are calculated by summing the 

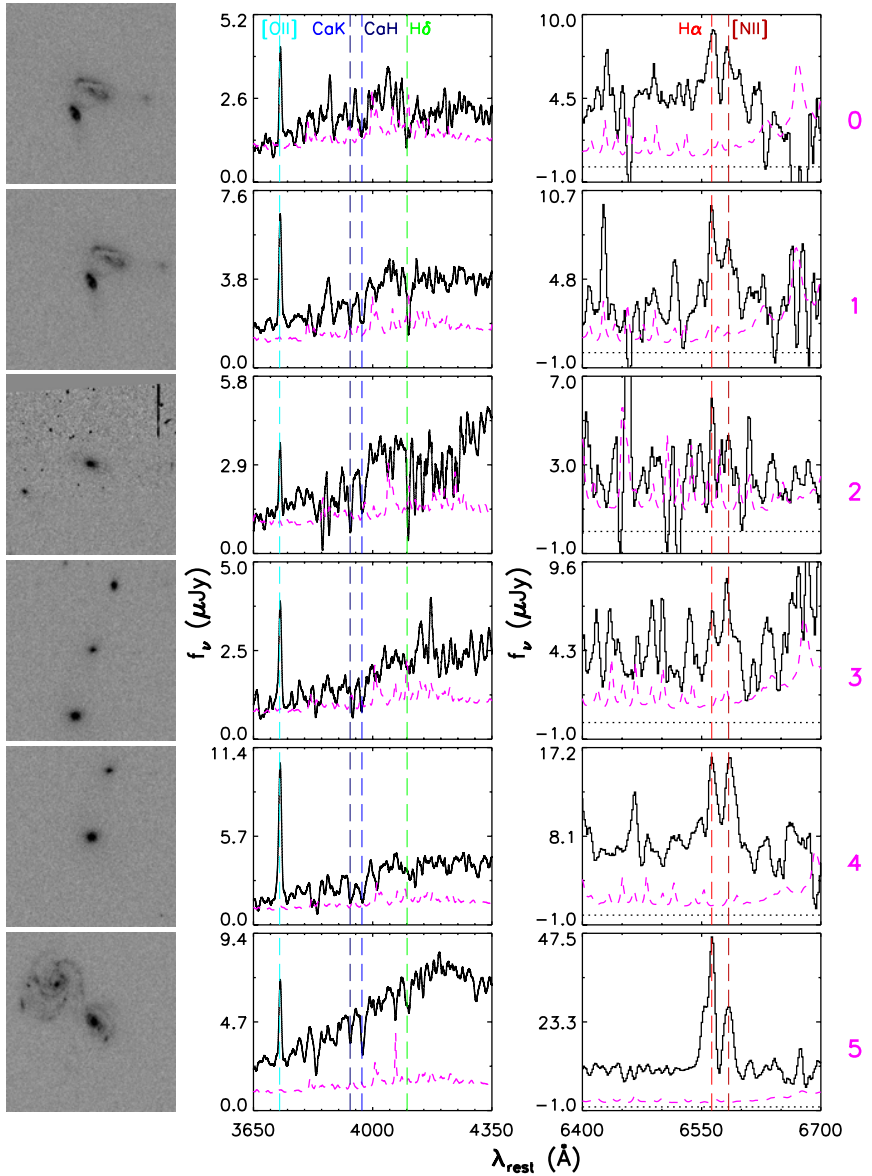

Figure 4. ACS $F 814 W$ postage stamps of each galaxy targeted with NIRSPEC as well as the associated rest-frame DEIMOS (left) and NIRSPEC (right) spectra of each galaxy. The galaxy number is indicated next to each spectrum. The DEIMOS spectra are smoothed with a Gaussian of FWHM 15 pixels (roughly $2.7 \AA$ rest frame at the redshift of the supercluster) and the NIRSPEC spectra are smoothed with a Gaussian of FWHM 1.7 pixels (roughly $2.7 \AA$ A rest frame). The error spectrum is plotted with a dashed line below each DEIMOS and NIRSPEC spectrum. All spectra are flux calibrated; however, no correction is made for internal extinction. For clarity, the DEIMOS spectra are plotted with the zero flux level at the bottom of the plot. Due to the low level of continuum emission in some of the NIRSPEC targets, a dotted line shows the zero flux level for each NIRSPEC spectrum. The long dashed lines show the locations of important spectral features. NIRSPEC targets $0-5$.

(A color version of this figure is available in the online journal.)

pixels along the spatial axis, using the best-fit Gaussian parameters to optimally weight (Horne 1986) the relative contribution from each pixel. In objects where the continuum had low S/N, but emission features were detected, the dispersion axis was collapsed only over the wavelength range containing the emission lines. For each science object, these one-dimensional spectra were combined into a single spectrum using an inverse variance weighted mean that preserved the overall flux calibration. Figures 4-8 show cutouts of the reduced NIRSPEC and DEIMOS/LRIS spectra of all 25 targets, as well as their associated ACS $F 814 W$ or LFC $i^{\prime}$ postage stamps.

\section{SPECTRAL LINE MEASUREMENTS}

\subsection{Equivalent Width and Emission Line Flux Ratios}

In each processed one-dimensional DEIMOS and NIRSPEC spectrum, we measure the EW of the [O II] and $\mathrm{H} \alpha$ nebular emission features. These EW measurements are useful because there exist well-measured correlations between the EW of the
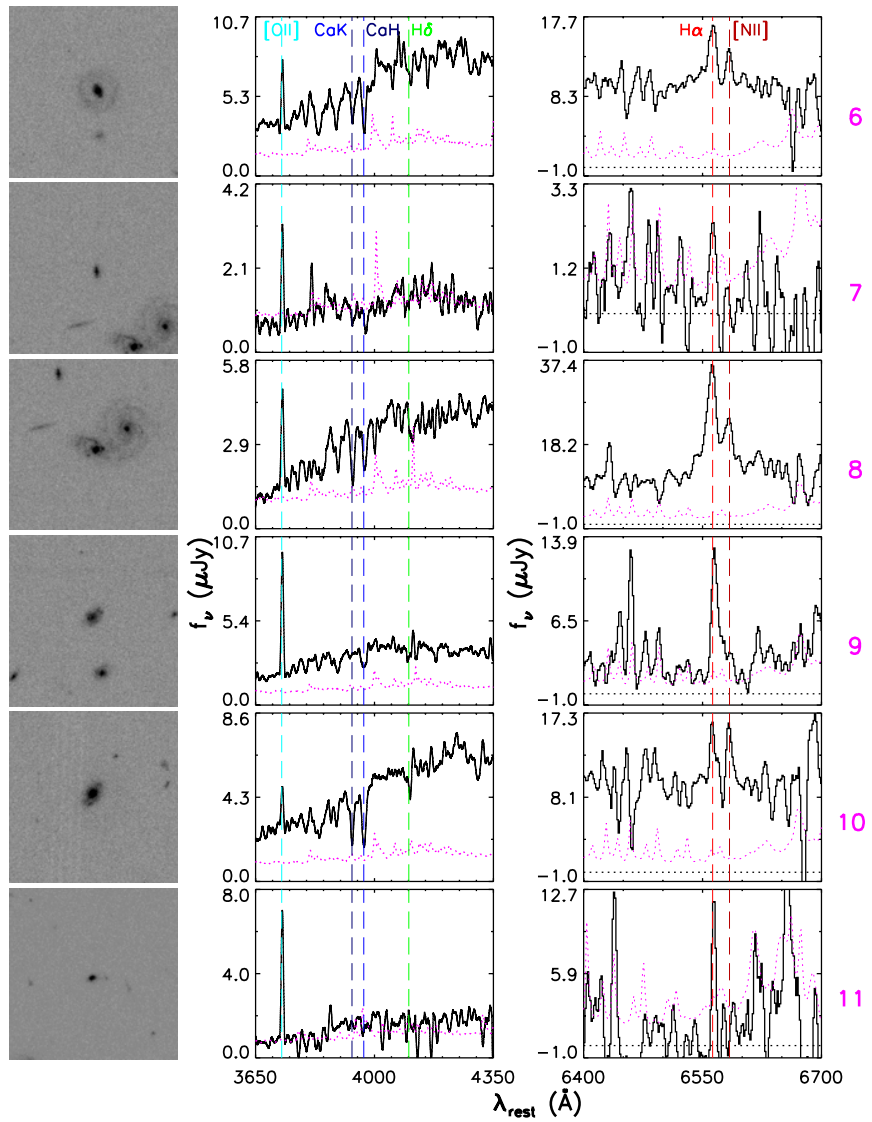

Figure 5. Same as in Figure 4. NIRSPEC targets 6-11.

(A color version of this figure is available in the online journal.)

$\mathrm{H} \alpha$ and [O II] lines in LINER-type and star-forming galaxies at low redshift, which generally are observed to be tighter than correlations observed in line luminosities (Y06).

In many galaxies, the properties of the dust affecting the stellar continuum are appreciably different from the nebular dust properties (see Calzetti et al. 1994; Calzetti 2001, and references therein). This differential extinction causes EWs to be slightly affected by dust abundance, decreasing $\operatorname{EW}(\mathrm{H} \alpha)$ by a factor of 1.25 and $\mathrm{EW}\left(\left[\mathrm{O}_{\mathrm{II}}\right]\right)$ by 1.36 using $E(B-V)=0.3$ and the Calzetti et al. (2000) reddening law. While the absolute numbers may be underestimated, we ignore this effect as any correlations that exist between the EW of the two features should remain essentially invariant with respect to dust properties.

The EW was measured in the rest frame using two different techniques: bandpass measurements and line-fitting techniques. Bandpass measurements were performed on all DEIMOS and NIRSPEC spectra by defining three bandpasses in the vicinity of the spectral feature adopted from Fisher et al. (1998) for [O II] and $\mathrm{Y} 06$ for the $\mathrm{H} \alpha$ and [N II] features. Line-fitting was performed on all spectra where emission lines were detected at a significance of greater than $3 \sigma$. For all measurements, spectra were fit by a double Gaussian model at fixed wavelength separation with a linear continuum. In NIRSPEC spectra, we tested the effect of including a third Gaussian (at fixed separation) to account for the weaker [N II] $\lambda 6548$ feature. In all cases, the effect on $\operatorname{EW}(\mathrm{H} \alpha)$ was extremely small $(\sim 5 \%)$ and consistent within the errors. For each spectrum, the EW measurement was chosen from the better of the two methods, typically depending on the feature $\mathrm{S} / \mathrm{N}$. The criteria for this choice as well as further details of the two methods are given 

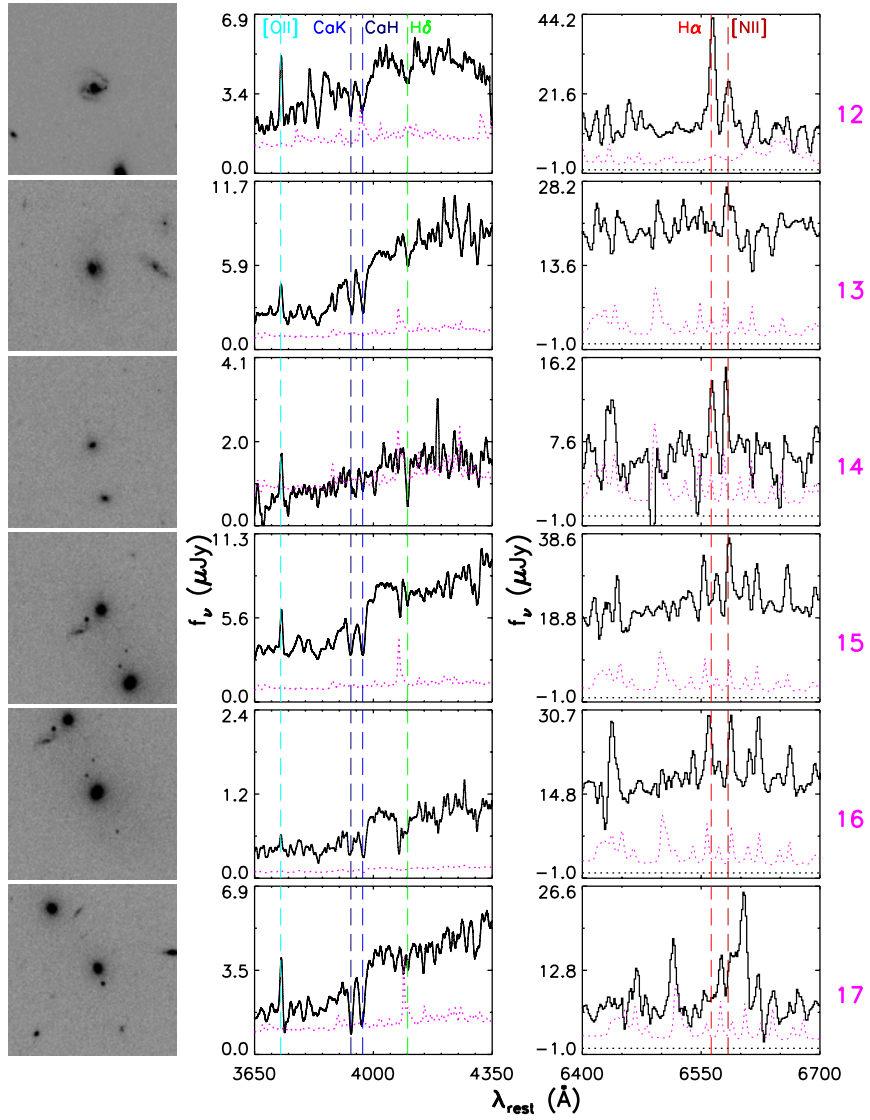

Figure 6. Same as in Figure 4. NIRSPEC targets 12-17. The spectrum for galaxy 16 obtained with LRIS (center panel) is not flux calibrated. The spectrum is smoothed with a Gaussian with a 2 pixel FWHM (roughly $3.4 \AA$ rest frame). (A color version of this figure is available in the online journal.)

in Appendix A. Note that, given our definition of EW in Appendix A (see Equation (A1)), the convention adopted in this paper is for positive EWs to correspond to features observed in emission and negative EWs to those observed in absorption.

While all [O II] EWs were measured in emission at significances much higher than $3 \sigma$, a subset $(\sim 20 \%)$ of our galaxies had $\mathrm{H} \alpha$ EWs that were measured in emission at significances less than $3 \sigma$. When analyzing line fluxes such galaxies are typically excluded (e.g., Tremonti et al. 2004; Kewley et al. 2006; Y06). However, our sample is comprised of galaxies that have $\left[\mathrm{O}_{\mathrm{II}}\right]$ in emission at very high significance. Our NIRSPEC observations are aimed at finding galaxies with low levels of $\mathrm{H} \alpha$ emission relative to their [O II] emission. Since galaxies with EWs measured at low significance are, by definition, weak $\mathrm{H} \alpha$ emitters (or absorbers), we include the $\mathrm{H} \alpha \mathrm{EW}$ measurements of all galaxies in our analysis regardless of the significance. Table 3 lists the $\mathrm{H} \alpha$ and [O II] EW measurements of all galaxies in our sample.

Emission line fluxes of the [O II], $\mathrm{H} \alpha$, and [N II] features were calculated using the same two methods as those used for EW measurements. For all galaxies in our sample, we used the same method to determine the line flux as was used for that galaxy's EW measurement. The bandpasses chosen for all features were identical to those used for measuring EWs. While we include EW measurements detected in emission at a significance less than $3 \sigma$ in our analyses, we typically do not include low significance measurements of line fluxes. The two exceptions are the $[\mathrm{N}$ II] measurement of galaxies 11 and 19 , for which the
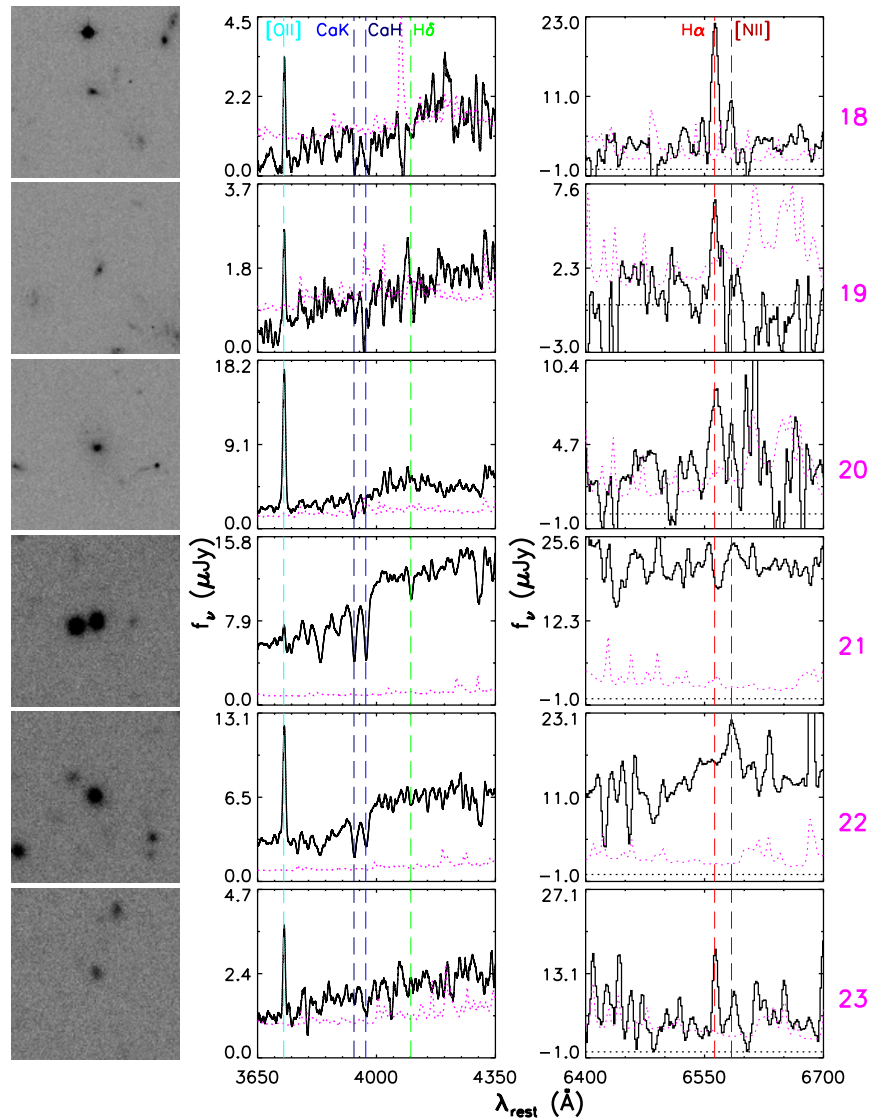

Figure 7. Same as in Figure 4. NIRSPEC targets 18-23. LFC $i^{\prime}$ postage stamps are used for galaxies 21-23 due to the lack of ACS data in the RX J1821 field. (A color version of this figure is available in the online journal.)
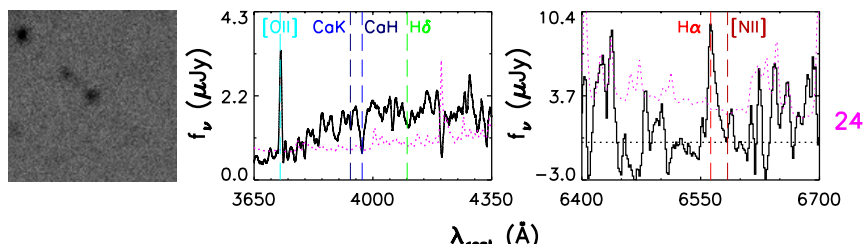

Figure 8. Same as in Figure 4. NIRSPEC target 24. An LFC $i^{\prime}$ postage stamp is used for galaxy 24 due to the lack of ACS data in the RX J1821 field. (A color version of this figure is available in the online journal.)

line is detected at a significance of approximately $2 \sigma$. In these cases, the $\left[\mathrm{N}_{\mathrm{II}}\right]$ line can be clearly seen in the two-dimensional spectrum, but the formal error (due to bright airglow lines in the vicinity of the detection) places the measurement at a significance of less than $3 \sigma$. The classification of these galaxies does not depend on this choice. For all other line fluxes that were detected at a significance less than $3 \sigma(5 / 25 \mathrm{H} \alpha$ lines; $3 / 25$ [N II] lines), $3 \sigma$ line fluxes were adopted as the formal upper bound. These upper limits allowed us to classify the one galaxy for which the $\mathrm{H} \alpha$ feature was detected at a significance greater than $3 \sigma$ but for which the $[\mathrm{N} \mathrm{II]} \mathrm{line} \mathrm{was} \mathrm{not} \mathrm{(galaxy} \mathrm{7),} \mathrm{and} \mathrm{the}$ three galaxies $(13,15$, and 21$)$ for which the [N II] feature was detected at high significance but $\mathrm{H} \alpha$ was not. The two galaxies (16 and 17) where neither line was detected at a significance greater than $3 \sigma$ are excluded from emission-line ratio analyses and are discussed further in Sections 5.2 and 5.3. Table 3 lists the $\mathrm{H} \alpha$ and $\left[\mathrm{N}_{\mathrm{II}}\right]$ line fluxes of all galaxies in our sample. 
Table 3

Spectral Properties of the NIRSPEC Sample

\begin{tabular}{|c|c|c|c|c|c|c|c|c|}
\hline ID & Galaxy Number & $z$ & $\begin{array}{c}\mathrm{EW}([\mathrm{O} \mathrm{II}])^{\mathrm{a}} \\
(\AA)\end{array}$ & $\begin{array}{c}\mathrm{EW}(\mathrm{H} \alpha)^{\mathrm{a}} \\
(\AA)\end{array}$ & $\begin{array}{c}\text { Class } \operatorname{EW}([\mathrm{O} \mathrm{II}]) / \\
\operatorname{EW}(\mathrm{H} \alpha)\end{array}$ & $\begin{array}{c}F(\mathrm{H} \alpha) \\
\left(\times 10^{-18} \mathrm{erg} \mathrm{s}^{-1} \mathrm{~cm}^{-2}\right)\end{array}$ & $\begin{array}{c}F([\mathrm{~N} \mathrm{II}]) \\
\left(\times 10^{-18} \mathrm{erg} \mathrm{s}^{-1} \mathrm{~cm}^{-2}\right)\end{array}$ & $\begin{array}{c}\text { Class } F([\mathrm{~N} \mathrm{II}]) / \\
F(\mathrm{H} \alpha)\end{array}$ \\
\hline J160344+432429 & 0 & 0.9023 & $18.8 \pm 1.7$ & $14.8 \pm 2.1$ & Low & $21.8 \pm 2.9$ & $17.5 \pm 2.6$ & LINER/Seyfert \\
\hline $\mathrm{J} 160344+432428$ & 1 & 0.9024 & $22.5 \pm 1.3$ & $13.3 \pm 1.8$ & Low & $18.9 \pm 2.1$ & $13.4 \pm 2.1$ & LINER/Seyfert \\
\hline $\mathrm{J} 160345+432419$ & 2 & 0.8803 & $10.7 \pm 1.3$ & $6.1 \pm 1.7$ & Low & $8.1 \pm 2.0$ & $6.5 \pm 1.9$ & LINER/Seyfert \\
\hline $\mathrm{J} 160342+432406$ & 3 & 0.8986 & $31.6 \pm 1.7$ & $6.7 \pm 1.8$ & High & $12.7 \pm 1.6$ & $17.4 \pm 2.9$ & LINER/Seyfert \\
\hline $\mathrm{J} 160342+432403$ & 4 & 0.8959 & $58.6 \pm 1.4$ & $17.0 \pm 1.3$ & Low & $44.5 \pm 2.2$ & $53.2 \pm 3.3$ & LINER/Seyfert \\
\hline $\mathrm{J} 160330+432208$ & 5 & 0.8983 & $16.1 \pm 0.8$ & $45.8 \pm 1.1$ & Low & $173.0 \pm 3.6$ & $85.0 \pm 2.9$ & Star forming \\
\hline $\mathrm{J} 160329+432204$ & 6 & 0.9045 & $8.7 \pm 1.0$ & $8.2 \pm 1.1$ & Low & $31.4 \pm 4.1$ & $13.3 \pm 2.6$ & Star forming \\
\hline $\mathrm{J} 160416+431021$ & 7 & 0.8990 & $17.4 \pm 2.3$ & $38.6 \pm 14.3$ & Low & $6.4 \pm 2.1$ & $<3.7^{\mathrm{b}}$ & Star forming \\
\hline $\mathrm{J} 160416+431017$ & 8 & 0.8999 & $17.8 \pm 1.4$ & $33.3 \pm 1.7$ & Low & $119.0 \pm 4.8$ & $38.4 \pm 3.1$ & Star orming \\
\hline $\mathrm{J} 160404+432445$ & 9 & 0.9017 & $31.5 \pm 0.9$ & $41.8 \pm 4.2$ & Low & $35.6 \pm 2.9$ & $4.5 \pm 1.5$ & Star forming \\
\hline $\mathrm{J} 160403+432436$ & 10 & 0.9015 & $10.2 \pm 1.9$ & $3.2 \pm 0.3$ & High & $18.0 \pm 3.4$ & $20.7 \pm 2.4$ & LINER/Seyfert \\
\hline $\mathrm{J} 160429+431956$ & 11 & 0.9185 & $55.3 \pm 1.9$ & $52.3 \pm 16.3$ & Low & $24.8 \pm 5.6$ & $6.1 \pm 3.8$ & Star forming \\
\hline $\mathrm{J} 160428+431953$ & 12 & 0.9198 & $9.0 \pm 1.1$ & $25.4 \pm 1.8$ & Low & $102.0 \pm 6.9$ & $55.5 \pm 6.0$ & Star forming \\
\hline $\mathrm{J} 160406+431542$ & 13 & 0.8674 & $9.9 \pm 0.9$ & $0.1 \pm 0.7$ & High & $<14.3^{\mathrm{b}}$ & $10.6 \pm 2.4$ & LINER/Seyfert \\
\hline $\mathrm{J} 160407+431539$ & 14 & 0.8676 & $23.7 \pm 3.3$ & $7.2 \pm 2.5$ & Low & $19.4 \pm 6.1$ & $12.3 \pm 4.1$ & LINER/Seyfert \\
\hline $\mathrm{J} 160426+431423$ & 15 & 0.8676 & $7.9 \pm 0.6$ & $0.4 \pm 0.7$ & High & $<16.5^{\mathrm{b}}$ & $14.1 \pm 3.0$ & LINER/Seyfert \\
\hline $\mathrm{J} 160426+431419$ & 16 & 0.8658 & $6.3 \pm 0.7$ & $1.6 \pm 1.1$ & High & $<22.0^{\mathrm{b}}$ & $<27.0^{\mathrm{b}}$ & Ambiguous \\
\hline $\mathrm{J} 160427+431501$ & 17 & 0.8601 & $6.3 \pm 0.7$ & $0.1 \pm 1.6$ & High & $<8.1^{\mathrm{b}}$ & $<15.7^{b}$ & Ambiguous \\
\hline $\mathrm{J} 160426+431439$ & 18 & 0.8710 & $56.7 \pm 6.1$ & $41.8 \pm 6.0$ & Low & $64.5 \pm 5.2$ & $23.4 \pm 4.6$ & Star forming \\
\hline $\mathrm{J} 160406+431825$ & 19 & 0.9189 & $50.9 \pm 4.5$ & $60.2 \pm 14.6$ & Low & $28.4 \pm 5.5$ & $13.4 \pm 5.1$ & Star forming \\
\hline $\mathrm{J} 160406+431809$ & 20 & 0.9195 & $73.7 \pm 1.4$ & $14.1 \pm 3.8$ & High & $29.7 \pm 3.6$ & $15.8 \pm 3.9$ & Star forming \\
\hline $\mathrm{J} 182110+682350$ & 21 & 0.7960 & $2.6 \pm 0.3$ & $0.3 \pm 0.3$ & High & $<20.0^{\mathrm{b}}$ & $23.3 \pm 4.1$ & LINER/Seyfert \\
\hline $\mathrm{J} 182108+682329$ & 22 & 0.8134 & $26.9 \pm 0.5$ & $1.9 \pm 0.5$ & High & $11.0 \pm 2.6$ & $44.4 \pm 3.3$ & LINER/Seyfert \\
\hline $\mathrm{J} 182121+682715$ & 23 & 0.8092 & $17.3 \pm 1.5$ & $20.6 \pm 3.5$ & Low & $37.8 \pm 6.0$ & $18.1 \pm 4.8$ & Star forming \\
\hline $\mathrm{J} 182123+682714$ & 24 & 0.8093 & $24.2 \pm 2.0$ & $39.9 \pm 10.0$ & Low & $32.9 \pm 3.9$ & $8.9 \pm 2.9$ & Star forming \\
\hline
\end{tabular}

Notes.

${ }^{\text {a }}$ Measured in the rest frame.

b $3 \sigma$ upper limit.

\subsection{Absolute Flux Measurements}

\subsubsection{DEIMOS Flux Calibration}

Absolute flux calibration of the Cl1604 DEIMOS data was obtained in a manner nearly identical to that of Lem09. Absolute flux calibration of the RX J1821 DEIMOS data was not performed as the photometry is less accurate (L09), and no HST ACS data exist in the field. The four NIRSPEC targets in this field are, therefore, excluded from analyses involving absolute flux measurements.

The DEIMOS spectrum of each Cl1604 member was response corrected using the generalized DEIMOS response function ${ }^{5}$ and checked for accuracy and precision using the methods of Lem09. An average slit throughput of $\omega_{\text {slit }}=0.37$ was adopted for all DEIMOS absolute flux measurements in order to minimize the observed systematic offset between the spectral and photometric magnitudes. This value matches the simulated slit-loss of a target slightly off-center from the 1" slits with a half-light radius of $r_{h}=00^{\prime} 18$ in $0{ }^{\prime \prime} 8$ seeing (Figure 4 of Lem09), reasonable values given our observing conditions and the ACS $F 814 W$ half-light radii of the galaxies observed.

The spectral magnitude, $i^{\prime}$ spec, is compared to our LFC photometry (see G08 for details) in Figure 9. The rms scatter of the spectral magnitudes is 0.48 mag, resulting in a $\sim 45 \%$ uncertainty in any absolute flux measurement. While there is little systematic bias, on average, between the spectral magnitudes and the LFC $i^{\prime}$ magnitudes, there does exist a noticeable trend of decreasing $i^{\prime}{ }_{\text {spec }}-i^{\prime}$ with increasing $i^{\prime}$ magnitude (see bottom

\footnotetext{
5 http://www.ucolick.org/ ripisc/results.html
}

panel of Figure 9). However, the NIRSPEC targets are primarily intermediate brightness cluster members $\left(i^{\prime} \sim 21.5-22.5\right)$ and comparisons between the spectral and photometric magnitudes at these magnitudes result in a distribution consistent with no systematic bias. We, therefore, ignore this bias and adopt an absolute uncertainty of $45 \%$ in any DEIMOS flux measurements, resulting purely from the rms scatter in the measured magnitudes.

\subsubsection{NIRSPEC Flux Calibration}

The two standards observed with NIRSPEC, HD105601 (A2) and HD203856 (A0), were chosen from the because of their similar spectral and luminosity class to $\alpha \mathrm{Lyr}$ and their low airmass at evening and morning twilight. Analysis of the count rates of the two standard stars resulted in observed variations of $\sim 20 \%$ between the two nights, but stable conditions during each individual night. Because HD203856 has an identical spectral class to $\alpha$ Lyr, absolute photometry was determined by scaling the spectrum of $\alpha$ Lyr (Colina et al. 1996) by the HD203856 Two Micron All Sky Survey (2MASS; Skrutskie et al. 2006) $J$-band magnitude of $6.896 \pm 0.023$. The scaled $\alpha$ Lyr spectrum was divided by the extinction-corrected composite spectrum of HD203856 ${ }^{6}$ created from observations of the standard star taken on each night.

Our flux calibration method implicitly involves a slit-loss correction for a point source (HD203856) under our observing conditions and will underpredict the slit-loss for a source with

\footnotetext{
6 Extinction correction was performed by correcting for the known $J$-band atmospheric extinction on Mauna Kea extinction, http://www2.keck.hawaii.edu/inst/nirc/exts.html.
} 

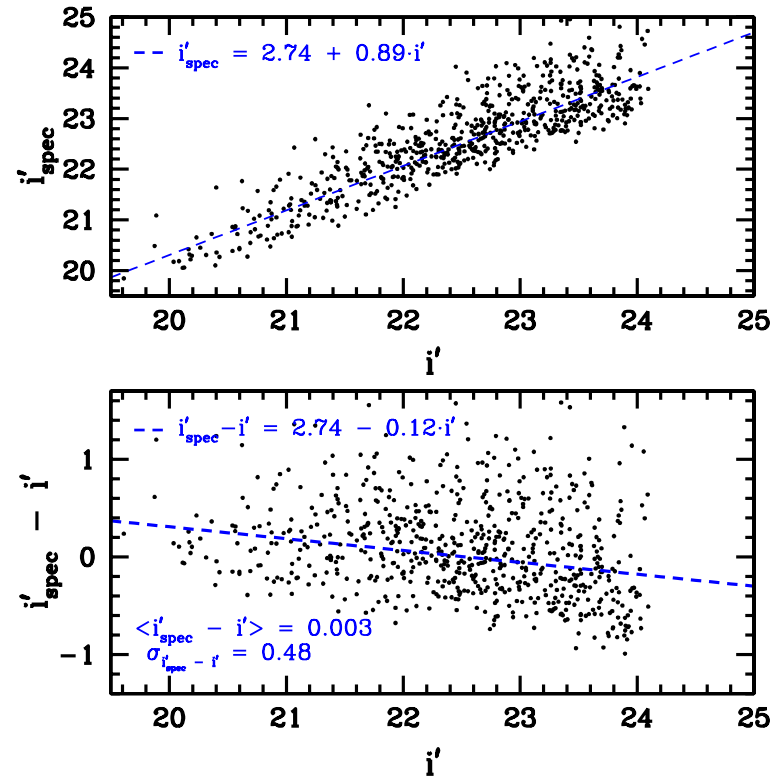

Figure 9. Top panel: LFC SDSS calibrated $i^{\prime}$ magnitudes $\left(i^{\prime}\right)$ plotted as a function of slit-loss corrected DEIMOS spectral $i^{\prime}$ magnitudes $\left(i_{\text {spec }}\right.$; see Section 4.2.1) for all members in the Cl1604 supercluster with high-quality $(Q \geqslant 3)$ spectra that fell near the middle of the slit and were not photometrically flagged. Bottom panel: the difference in the spectral and LFC $i^{\prime}$ magnitudes as a function of $i^{\prime}$ for the same galaxies. The best-fit relations are overplotted. The large scatter in both panels represent real uncertainties in flux calibration of the data. An average slit throughput of $\omega_{\text {slit }}=0.37$ was adopted for all spectra. This correction reproduces well the LFC $i^{\prime}$ magnitudes on average. This throughput is optimized for the average $i^{\prime}$ magnitude of our NIRSPEC galaxies $\left(i^{\prime} \sim 22.2\right)$ to avoid the magnitude dependent bias in the flux calibration that arises from the size-magnitude relationship.

(A color version of this figure is available in the online journal.)

finite intrinsic angular extent. However, based on simulations similar to those performed in Section 4.2.1, we find maximal slit-loss of only $10 \%-15 \%$ relative to a point source for galaxies with a 0!3 half-light radius in our observing conditions. No correction is applied for this effect as we cannot accurately quantify the $J$-band half-light radius of each NIRSPEC target. Relative measurements (e.g., EWs or ratios of line fluxes) are unaffected by such losses. For absolute flux measurements, any losses of this nature will result in underestimates of the true flux. The error associated with each line luminosity is then a quadrature sum of the random errors discussed in Appendix A and the systematic error associated with either the DEIMOS (for [O II] line luminosities) or NIRSPEC (for $\mathrm{H} \alpha$ line luminosities) flux calibration.

\subsubsection{Extinction Corrections}

An additional uncertainty involved in comparing absolute flux measurements is due to internal extinction. Internal extinction corrections have been made using a variety of methodologies (e.g., Kennicutt 1983; Kaufman et al. 1987; Wang \& Heckman 1996; Jansen et al. 2001; Kewley et al. 2002, 2004; Kauffmann et al. 2003a; Buat et al. 2005; Moustakas et al. 2006; Rudnick et al. 2006; Weiner et al. 2007). While such methods have been shown to be reliable for statistical samples of galaxies (though with differing levels of scatter, see Argence \& Lamareille 2009 for a review), applying corrections to absolute line luminosities of an individual galaxy may lead to significant biases.

For this data, we attempt dust corrections based on (1) the luminosity of the $\mathrm{H} \alpha$ line relative to the $24 \mu \mathrm{m}$ luminosity in those NIRSPEC targets detected in the MIPS data, (2) the absolute $B$-band magnitude, and (3) extinction values derived from spectral energy distribution (SED) fitting to broadband photometry $\left(r^{\prime} i^{\prime} z^{\prime} K_{s}+\mathrm{IRAC}\right)$. The details of each extinction correction method as well as the range of extinction values derived from each method are given in Appendix B. For all extinction corrections, we assume a reddening curve, $k^{\prime}(\lambda)$, parameterized by Calzetti et al. (2000), a total $V$-band obscuration of $R^{\prime}{ }_{V}=4.05 \pm 0.80$, and a value of $E_{S}(B-V)=$ $(0.44 \pm 0.03) E(B-V)$, where $E_{S}(B-V)$ is the extinction of early-type stellar continua and $E(B-V)$ is the nebular extinction. The Calzetti reddening curve is adopted because it is valid over our range of extinction values and is known to properly characterize the dust properties of galaxies at $z<1$ (Caputi et al. 2008; Conroy 2009). Further details regarding each extinction correction method as well as the range of extinction values derived from each method are given in Appendix B.

Although the mean extinction values derived by the above three methods are consistent at the $1 \sigma$ level (see Appendix B), the $E(B-V)$ of an individual galaxy using each of the three methods frequently varied at levels exceeding $3 \sigma$. Therefore, when determining intrinsic line luminosities from our NIRPSEC data, we choose not to correct an individual galaxy using any of the above methods and instead adopt a constant $E(B-V)=0.3$ for all galaxies. This value was chosen because it is consistent with the observed mean extinctions found by all three methods (see Appendix B) and is well motivated from the observed extinctions in local samples that span a large range of galaxy types and luminosities (Nearby Field Galaxies Survey (NFGS): Jansen 2000; Moustakas \& Kennicutt 2006). In Section 5.4, we discuss the implications of choosing a constant extinction value rather than one of the above three methods. Table 4 lists the observed fluxes and luminosities of the $\mathrm{H} \alpha$ and [O II] lines of our sample, as well as the extinction-corrected luminosities.

\section{RESULTS AND DISCUSSION}

The $[\mathrm{O}$ II $] \lambda 3727$ doublet can originate from many processes that include, but are not limited to, star formation, such as, e.g., galactic shocks (Heckman 1980; Dopita \& Sutherland 1995; Veilleux et al. 1995), cooling flows (Heckman 1981; Heckman et al. 1989), photoionization by hot stars (Terlevich \& Melnick 1985; Filippenko \& Terlevich 1992; Shields 1992), post-asymptotic giant branch stars (Binette et al. 1994; Taniguchi et al. 2000), or emission from an AGN (Ferland \& Netzer 1983; Filippenko \& Halpern 1984; Ho et al. 1993; Filippenko 2003; Kewley et al. 2006). While these processes can also result in residual levels of $\mathrm{H} \alpha$ flux, in general, classification that relies on the strength of the $\mathrm{H} \alpha$ feature has been shown to be much less sensitive to contamination by these processes (Kauffmann et al. 2003b; Y06). In this section, we consider the relative strengths of [O II] and $\mathrm{H} \alpha$, as well as the relative strengths of [N II] and $\mathrm{H} \alpha$ in order to probe the nature of the dominant source of ionizing flux in high-redshift cluster galaxies. Following this, we investigate the other properties of galaxies dominated by LINER/Seyfert emission and those classified as star forming to determine their role in the context of galaxy evolution.

\subsection{H $\alpha$ and [O II] Equivalent Width Properties of the NIRSPEC Sample}

In Figure 10, we plot the rest-frame EW of [O II] against the rest-frame $\mathrm{EW}$ of $\mathrm{H} \alpha$ for all 25 galaxies in our sample. Due to the small number of galaxies observed with NIRSPEC, we separate populations that have [O II] EWs greater than expected, given 
Table 4

Star Formation Properties of the C11604 NIRSPEC Sample

\begin{tabular}{|c|c|c|c|c|c|c|c|c|c|c|}
\hline ID & Galaxy & $z$ & $F(\mathrm{H} \alpha)$ & $F([\mathrm{O}$ II $])$ & $\begin{array}{c}L(\mathrm{H} \alpha)_{\mathrm{obs}} \\
\left(10^{40} \mathrm{erg} \mathrm{s}^{-1}\right)\end{array}$ & $\begin{array}{c}L([\mathrm{O} \text { II }])_{\mathrm{obs}} \\
\left(10^{40} \mathrm{erg} \mathrm{s}^{-1}\right)\end{array}$ & $\begin{array}{c}L(\mathrm{H} \alpha)^{\mathrm{a}}{ }_{\text {corr }} \\
\left(10^{40} \mathrm{erg} \mathrm{s}^{-1}\right)\end{array}$ & $\begin{array}{c}\operatorname{SFR}(\mathrm{H} \alpha)_{\text {corr }}^{\mathrm{a}} \\
\left(M_{\odot} \mathrm{yr}^{-1}\right)\end{array}$ & $\begin{array}{c}L(\mathrm{O} \text { II })_{\text {corr }}^{\mathrm{a}} \\
\left(10^{40} \mathrm{erg} \mathrm{s}^{-1}\right)\end{array}$ & $\begin{array}{l}\mathrm{SFR}([\mathrm{O} \mathrm{II}])_{\text {corr }}^{\mathrm{a}} \\
\quad\left(M_{\odot} \mathrm{yr}^{-1}\right) \\
\end{array}$ \\
\hline $\mathrm{J} 160344+432429$ & 0 & 0.9023 & $21.8 \pm 2.9$ & $11.4 \pm 5.1$ & $8.7 \pm 1.2$ & $4.6 \pm 2.1$ & $22.2 \pm 6.3$ & $1.7 \pm 0.5$ & $23.2 \pm 11.9$ & $1.6 \pm 1.0$ \\
\hline $\mathrm{J} 160344+432428$ & 1 & 0.9024 & $18.9 \pm 2.1$ & $20.8 \pm 9.3$ & $7.7 \pm 0.8$ & $8.5 \pm 3.8$ & $19.2 \pm 5.2$ & $1.5 \pm 0.4$ & $42.7 \pm 21.8$ & $3.0 \pm 1.8$ \\
\hline $\mathrm{J} 160345+432419$ & 2 & 0.8803 & $8.1 \pm 2.0$ & $8.9 \pm 4.0$ & $3.1 \pm 0.7$ & $3.4 \pm 1.5$ & $7.8 \pm 2.7$ & $0.6 \pm 0.2$ & $17.1 \pm 8.8$ & $1.2 \pm 0.7$ \\
\hline $\mathrm{J} 160342+432406$ & 3 & 0.8986 & $12.7 \pm 1.6$ & $11.6 \pm 5.2$ & $5.1 \pm 0.6$ & $4.7 \pm 2.1$ & $12.8 \pm 3.6$ & $1.0 \pm 0.3$ & $23.6 \pm 12.0$ & $1.6 \pm 1.0$ \\
\hline $\mathrm{J} 160342+432403$ & 4 & 0.8959 & $44.5 \pm 2.2$ & $43.6 \pm 19.3$ & $17.7 \pm 0.9$ & $17.4 \pm 7.7$ & $44.5 \pm 11.2$ & $3.5 \pm 0.9$ & $87.9 \pm 44.6$ & $6.1 \pm 3.6$ \\
\hline $\mathrm{J} 160330+432208$ & 5 & 0.8983 & $173.0 \pm 3.6$ & $18.1 \pm 8.0$ & $69.5 \pm 1.5$ & $7.3 \pm 3.2$ & $174.2 \pm 43.3$ & $13.8 \pm 3.4$ & $36.7 \pm 18.6$ & $2.5 \pm 1.5$ \\
\hline $\mathrm{J} 160329+432204$ & 6 & 0.9045 & $31.4 \pm 4.1$ & $17.1 \pm 7.6$ & $12.8 \pm 1.7$ & $7.0 \pm 3.1$ & $32.2 \pm 9.0$ & $2.5 \pm 0.7$ & $35.3 \pm 18.0$ & $2.5 \pm 1.4$ \\
\hline $\mathrm{J} 160416+431021$ & 7 & 0.8999 & $6.4 \pm 2.1$ & $9.8 \pm 4.3$ & $2.6 \pm 0.8$ & $3.9 \pm 1.7$ & $6.4 \pm 2.6$ & $0.5 \pm 0.2$ & $19.8 \pm 10.1$ & $1.4 \pm 0.8$ \\
\hline J160416+431017 & 8 & 0.8999 & $119.0 \pm 4.8$ & $13.9 \pm 6.2$ & $48.0 \pm 1.9$ & $5.6 \pm 2.5$ & $120.4 \pm 30.2$ & $9.5 \pm 2.4$ & $28.4 \pm 14.5$ & $2.0 \pm 1.2$ \\
\hline J160404+432445 & 9 & 0.9017 & $35.6 \pm 2.9$ & $30.4 \pm 13.4$ & $14.4 \pm 1.2$ & $12.3 \pm 5.4$ & $36.2 \pm 9.4$ & $2.9 \pm 0.7$ & $62.1 \pm 31.5$ & $4.3 \pm 2.5$ \\
\hline $\mathrm{J} 160403+432436$ & 10 & 0.9015 & $18.0 \pm 3.4$ & $8.1 \pm 3.7$ & $7.3 \pm 1.4$ & $3.3 \pm 1.5$ & $18.3 \pm 5.7$ & $1.4 \pm 0.5$ & $16.6 \pm 8.5$ & $1.2 \pm 0.7$ \\
\hline J160429+431956 & 11 & 0.9185 & $24.8 \pm 5.6$ & $23.1 \pm 10.2$ & $10.5 \pm 2.4$ & $9.8 \pm 4.3$ & $26.3 \pm 8.8$ & $2.1 \pm 0.7$ & $49.5 \pm 25.1$ & $3.4 \pm 2.0$ \\
\hline $\mathrm{J} 160428+431953$ & 12 & 0.9198 & $102.0 \pm 6.9$ & $11.8 \pm 5.3$ & $43.4 \pm 2.9$ & $5.0 \pm 2.3$ & $108.9 \pm 27.9$ & $8.6 \pm 2.2$ & $25.4 \pm 13.1$ & $1.8 \pm 1.1$ \\
\hline $\mathrm{J} 160406+431542$ & 13 & 0.8674 & $<14.3^{\mathrm{b}}$ & $9.8 \pm 4.4$ & $<5.3^{\mathrm{b}}$ & $3.6 \pm 1.6$ & $<13.2^{\mathrm{b}}$ & $<1.0^{\mathrm{b}}$ & $18.3 \pm 9.4$ & $1.3 \pm 0.8$ \\
\hline J160407+431539 & 14 & 0.8676 & $19.4 \pm 6.1$ & $4.9 \pm 2.4$ & $7.2 \pm 2.3$ & $1.8 \pm 0.9$ & $17.9 \pm 7.2$ & $1.4 \pm 0.6$ & $9.1 \pm 5.1$ & $0.6 \pm 0.4$ \\
\hline $\mathrm{J} 160426+431423$ & 15 & 0.8676 & $<16.5^{\mathrm{b}}$ & $11.3 \pm 5.0$ & $<6.1^{\mathrm{b}}$ & $4.2 \pm 1.9$ & $<15.2^{\mathrm{b}}$ & $<1.2^{\mathrm{b}}$ & $21.0 \pm 10.7$ & $1.5 \pm 0.9$ \\
\hline $\mathrm{J} 160426+431419$ & 16 & 0.8658 & $<22.0^{\mathrm{b}}$ & $\ldots{ }^{c}$ & $<8.1^{\mathrm{b}}$ & $\ldots{ }^{c}$ & $<20.2^{\mathrm{b}}$ & $<1.6^{\mathrm{b}}$ & $\ldots{ }^{\mathrm{c}}$ & $\ldots{ }^{\mathrm{c}}$ \\
\hline $\mathrm{J} 160427+431501$ & 17 & 0.8601 & $<8.1^{\mathrm{b}}$ & $9.7 \pm 4.4$ & $<2.9^{\mathrm{b}}$ & $3.5 \pm 1.6$ & $<7.3^{\mathrm{b}}$ & $<0.6^{\mathrm{b}}$ & $17.7 \pm 9.1$ & $1.2 \pm 0.8$ \\
\hline J160426+431439 & 18 & 0.8710 & $64.5 \pm 5.2$ & $12.2 \pm 5.5$ & $24.0 \pm 1.9$ & $4.6 \pm 2.0$ & $60.2 \pm 15.7$ & $4.8 \pm 1.2$ & $23.0 \pm 11.8$ & $1.6 \pm 0.9$ \\
\hline J160406+431825 & 19 & 0.9189 & $28.4 \pm 5.5$ & $8.0 \pm 3.6$ & $12.0 \pm 2.4$ & $3.4 \pm 1.5$ & $30.3 \pm 9.5$ & $2.4 \pm 0.8$ & $17.2 \pm 8.9$ & $1.2 \pm 0.7$ \\
\hline J160406+431809 & 20 & 0.9195 & $29.7 \pm 3.6$ & $7.2 \pm 3.2$ & $12.6 \pm 1.5$ & $30.6 \pm 13.5$ & $31.7 \pm 8.7$ & $2.5 \pm 0.7$ & $154.4 \pm 78.3$ & $10.7 \pm 6.3$ \\
\hline
\end{tabular}

Notes.

${ }^{\text {a }}$ Corrected using a constant extinction of $E(B-V)=0.3$.

b $3 \sigma$ upper limit.

c No flux measurement was attempted for the LRIS object, due to the uncertainty in the flux calibration.

the relative strength of $\mathrm{H} \alpha$, from normal star-forming processes using the classification employed by Y06 for 55,000 galaxies at low redshift. The boundary, shown as a dashed line in Figure 10, is given by

$$
\mathrm{EW}([\mathrm{O} \mathrm{II}])=5 \mathrm{EW}(\mathrm{H} \alpha)-8 .
$$

Galaxies to the left of the line are classified as "high [O II]/H $\alpha$ "; galaxies to the right of the line are classified as "low [O II $] / \mathrm{H} \alpha$." In the low-redshift sample (Y06), there exist two populations that are well represented by $\log$ normal distributions, one centered at $\operatorname{EW}([\mathrm{O} \mathrm{II}]) \approx 10 \AA$ and $\operatorname{EW}(\mathrm{H} \alpha) \approx 14 \AA$ (their low-[O II $] / \mathrm{H} \alpha$ population) and one centered at $\mathrm{EW}([\mathrm{O} \mathrm{II}]) \approx$ $6 \AA$ and $\mathrm{EW}(\mathrm{H} \alpha) \approx 1 \AA$ (their high-[O II $] / \mathrm{H} \alpha$ population; see Figure 2 of Y06). The differences between these two populations at low redshift were generally due to the dominant emission mechanism present in each population. High-[O II $] / \mathrm{H} \alpha$ and red low-[O $\mathrm{II}] / \mathrm{H} \alpha$ populations of $\mathrm{Y} 06$ generally had emissionline ratios that were consistent with emission from a source other than star formation (i.e., a Seyfert or, more commonly, a LINER). Conversely, the blue low-[O II]/H $\alpha$ galaxies in this same study typically exhibited emission-line ratios consistent with normal star formation. Table 3 lists the EW class of each galaxy in our sample.

Although this bimodality in our data is blurred by several galaxies at or near the boundary between low and high [O II]/ $\mathrm{H} \alpha$, the nine galaxies in our main sample that have moderate levels of [O II ] emission $[\mathrm{EW}([\mathrm{O} \mathrm{II}]) \lesssim 10 \AA]$ have $\mathrm{H} \alpha$ EWs that span a factor of $\sim 300$. This suggests that there exist significantly different mechanisms generating the ionizing flux in the galaxies in the main sample. The filler sample all belong to the low[O II] $/ \mathrm{H} \alpha$ population and cover a much smaller dynamic range in this space. This result is not surprising as these galaxies have DEIMOS spectra that are indicative of normal star formation processes. While our data suggest an interpretation similar to

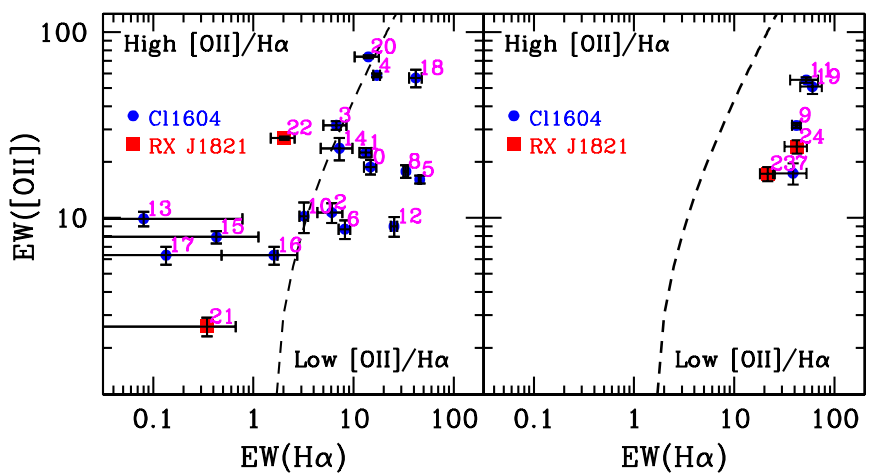

Figure 10. $\mathrm{EW}([\mathrm{OII}])$ vs. $\mathrm{EW}(\mathrm{H} \alpha)$ for the 19 galaxies in our main sample (left) and the 6 galaxies in our filler sample (right). As discussed in Section 4.1, we include the measured EWs of all galaxies regardless of the significance of the detection. All [O II] features have EWs that are detected in emission at $>3 \sigma$. The dividing line between "high $[\mathrm{O}$ II $] / \mathrm{H} \alpha$ " and "low $[\mathrm{O}$ II $] / \mathrm{H} \alpha$ " adopted from the low-redshift sample of Y06 is plotted as a dashed line. While the main sample covers a large dynamic range in this space, spanning 4 orders of magnitude in $\mathrm{H} \alpha \mathrm{EW}$ and approximately 2 orders of magnitude in [O II] EW, the filler sample are all low-[O II] $/ \mathrm{H} \alpha$ galaxies and are confined to a narrow region in this phase space. Galaxy symbols are coded by cluster membership and are labeled with galaxy numbers that correspond to the numbering in Tables $1-4$ and 7.

(A color version of this figure is available in the online journal.)

that of Y06, the mean absolute magnitude and rest-frame colors spanned by our sample differ appreciably from those in the Y06 sample. This difference may be important since the strength of the EW increases due to either (1) an increase in the luminosity of a spectral feature at a set continuum level or (2) a fainter broadband magnitude at a fixed line luminosity.

Differential changes in the stellar continuum levels can have a substantial effect on the observed correlations as the [O II] EW 


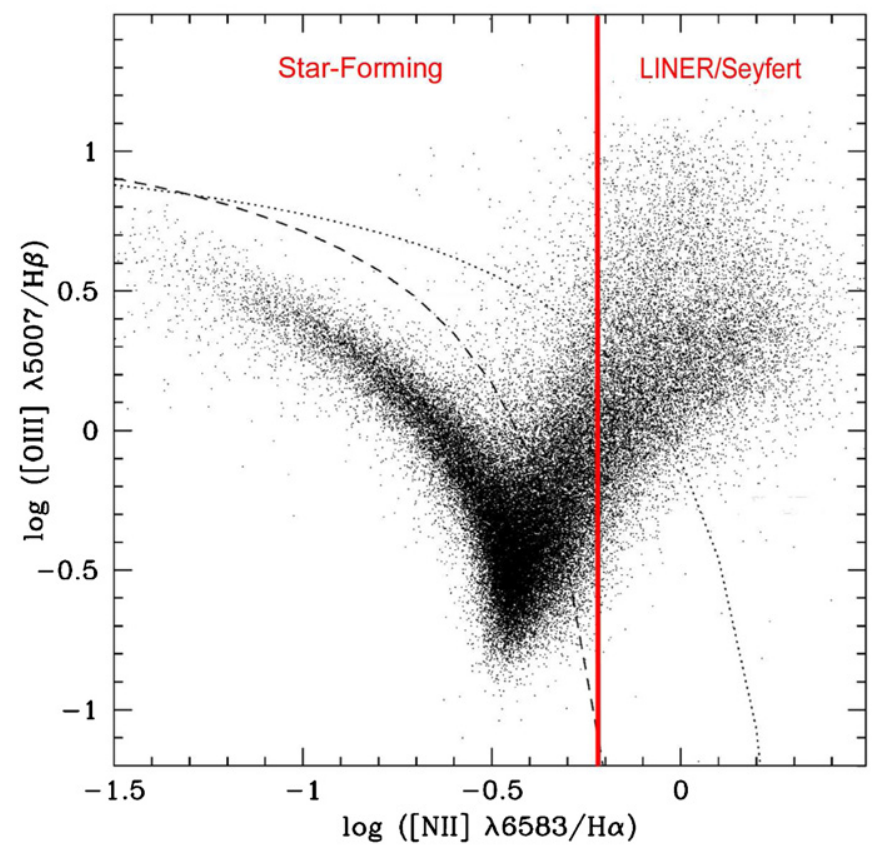

Figure 11. Figure adopted from Kauffmann et al. (2003b) showing the emission line flux ratios for $\sim 55,000$ SDSS galaxies used to discriminate the emission class of each galaxy. The dashed line denotes the dividing line between starforming galaxies and those with emission from a LINER or a Seyfert defined by Kauffmann et al. The dotted line shows the maximum boundary of a pure starburst galaxy from theoretical modeling performed by Kewley et al. (2001; i.e., the "extreme starburst line"). The region in between the two lines delineates galaxies with superpositions of star formation and LINER/Seyfert activity (i.e., TOs). The red solid vertical line shows our adopted boundary of $\log \left(F_{[\mathrm{NI}] / \mathrm{H} \alpha}\right)=-0.22$ between star-forming and LINER/Seyfert galaxies. Note that this boundary sets only a lower limit to the number of LINERs, Seyferts, and TOs in our sample, as there exist a significant number of galaxies in the SDSS sample that are inconsistent with pure star formation (mostly TOs) with $-0.4<\log \left(F_{[\mathrm{N}}\right.$ II] $\left./ \mathrm{H} \alpha\right)<-0.22$.

(A color version of this figure is available in the online journal.)

may be artificially inflated (in the sense of not being tied directly to the strength of a star formation episode) with respect to the $\mathrm{H} \alpha \mathrm{EW}$ in galaxies with $g^{\prime}-r^{\prime}$ colors. However, if we adopt observed frame $i^{\prime}-z^{\prime}$ or $i^{\prime}-K_{s}$ color as a proxy for the difference in continuum levels surrounding the $[\mathrm{O} \mathrm{II}]$ and $\mathrm{H} \alpha$ features, the $\mathrm{Cl1604}$ high-[O II]/H $\alpha$ population is only $\sim 0.1$ mag redder than the low-[O II $] / \mathrm{H} \alpha$ sample. Therefore, variations in stellar continua are not responsible for the observed difference between our low-[O II] $/ \mathrm{H} \alpha$ and high-[O II] $/ \mathrm{H} \alpha$ populations; instead they must reflect real variations in line luminosities. In the following sections, we explore the nature of that variation.

\subsection{Investigating the Dominant Source of Emission in the NIRSPEC Sample}

The three possible causes of variation in the observed luminosity of [O $\mathrm{II}]$ at a fixed $\mathrm{H} \alpha$ value are (1) the primary source of ionizing flux, (2) extinction, and (3) changes in metallicity. To investigate the first possibility, we utilize a modified version of the BPT diagrams (Baldwin et al. 1981; Veilleux \& Osterbrock 1987). Traditionally, diagrams that utilize the strength of the $\mathrm{H} \beta$ feature relative to [O III] and the strength of the $\mathrm{H} \alpha$ feature relative to a forbidden line (typically $6300 \AA$ [O I], [N II], or $6716 \AA$ + $6731 \AA[\mathrm{S} \mathrm{II}]$ due to their proximity to $\mathrm{H} \alpha$ ) are favored (see Figure 11). As we do not observe all the spectral features necessary for a full BPT analysis, we instead rely on a variation of this diagnostic to separate star-forming galaxies from LINERs and Seyferts. We use [N II] rather than [O I] or [S II] as [O I] is typically too weak to detect, and $[\mathrm{S}$ II] coincides with bright $\mathrm{OH}$ airglow lines at $\lambda>1.25 \AA$. In Figure 12, we plot our modified version of the BPT diagram for the main and filler samples. Only galaxies where [O II] and at least one other NIR emission line $(\mathrm{H} \alpha$ or $[\mathrm{N}$ II $]$ ) were detected at high significance are plotted.

Since we are collapsing the traditional $F([\mathrm{O} \mathrm{III}]) / F(\mathrm{H} \beta)$ (hereafter $F_{[\mathrm{O} \mathrm{III}] / \mathrm{H} \beta}$ ) ordinate of the BPT diagram, choosing a boundary along the $F([\mathrm{~N} I I]) / F(\mathrm{H} \alpha)$ (hereafter $\left.F_{[\mathrm{NII}] / \mathrm{H} \alpha}\right)$ axis that discriminates between galaxies dominated by emission from $\mathrm{H}$ II regions and those dominated by a LINER or Seyfert is somewhat ambiguous. The large range of metallicities and ionization parameters present in star-forming galaxies results in a wide range of observed $F_{[\mathrm{NI}] / \mathrm{H} \alpha}$ values. A study of nearly 100,000 SDSS galaxies by Kewley et al. (2006) found that this ratio varied from $\log \left(F_{[\mathrm{NII} / \mathrm{H} \alpha}\right) \approx-1.5$ in extremely metal-poor star-forming galaxies to $\log \left(F_{[\mathrm{NI}] / \mathrm{H} \alpha}\right) \approx-0.3$ in those with super-solar abundances. Without the additional information that $F_{\left[\mathrm{O}_{\text {III }}\right] / \mathrm{H} \beta}$ provides we divide star-forming galaxies from those dominated by a LINER or Seyfert component (referred hereafter as "LINER/Seyfert" galaxies) at $\log \left(F_{[\mathrm{NI}] / \mathrm{H} \alpha}\right)=-0.22$. This value reflects the maximal boundary of star-forming galaxies in the sample of Kauffmann et al. (2003b; see vertical line in Figure 11) and is similar to cuts used in other studies when $\mathrm{H} \beta$ and [O III] are weak or unobservable (e.g., Miller et al. 2003; Stasińska et al. 2006). With the adoption of $\log \left(F_{[\mathrm{NII}] / \mathrm{H} \alpha}\right)>$ -0.22 as our bound for LINER/Seyferts, we also include in this cut "transition objects" (hereafter TOs). These galaxies have ionizing flux that is a composite of emission from power-law sources, emission from old populations of metal-rich stars, and emission from H II regions (Ho et al. 1993; Kauffmann et al. 2003b; Kewley et al. 2006). Even though our cut will classify a galaxy as a LINER/Seyfert if as little as $20 \%$ of its overall ionizing flux originates from a power-law source, it is sufficient for our study that any such galaxy must have some contribution from processes that are not star forming.

The choice of $\log \left(F_{[\mathrm{NI}] / \mathrm{H} \alpha}\right)<-0.22$ as a bound for starforming galaxies will result in some galaxies that are not truly star forming being classified as such. This is especially true for galaxies with $\log \left(F_{[\mathrm{NII} / \mathrm{H} \alpha}\right)>-0.35$, where the "contamination" by power-law sources (primarily Seyferts and TOs) becomes significant (Stasińska et al. 2006; see also Figure 11). For our main sample (plotted in the left panel of Figure 12), no galaxies have $\log \left(F_{\mathrm{NII} / \mathrm{H} \alpha}\right)<-0.5$, suggesting that the contamination by LINER/Seyferts in galaxies classified as star forming is quite high. This conservative selection is necessary since we are attempting to show that [O $\mathrm{II}]$ emission in many of main sample galaxies cannot be solely attributed to starforming processes. Metal-enriched starbursts with unusually low-ionization parameters may exhibit $\log \left(F_{\mathrm{NII} / \mathrm{H} \alpha}\right)$ as high as $0.2-0.3$ (i.e., the "extreme starburst line" in Figure 11; Kewley et al. 2001), thus contaminating our LINER/Seyfert sample; however, such galaxies are somewhat rare at $z \sim 0.1$ and likely extremely so at $z \sim 1$ (Tremonti et al. 2004; Kewley \& Ellison 2008; Liu et al. 2008; Lara-López et al. 2009; Pérez-Montero et al. 2009).

Of the 19 galaxies in our main (priority = 1) sample, 11 ( $\sim 58 \%)$ are classified LINER/Seyfert, with several of the "starforming" galaxies lying within $1 \sigma$ of the dividing line. This result is significant, as priority 1 galaxies make up a large subsection of the population in both Cl1604 and RX J1821. In the absence of NIR spectroscopy, all of the priority 1 galaxies would be considered star forming, significantly skewing the 

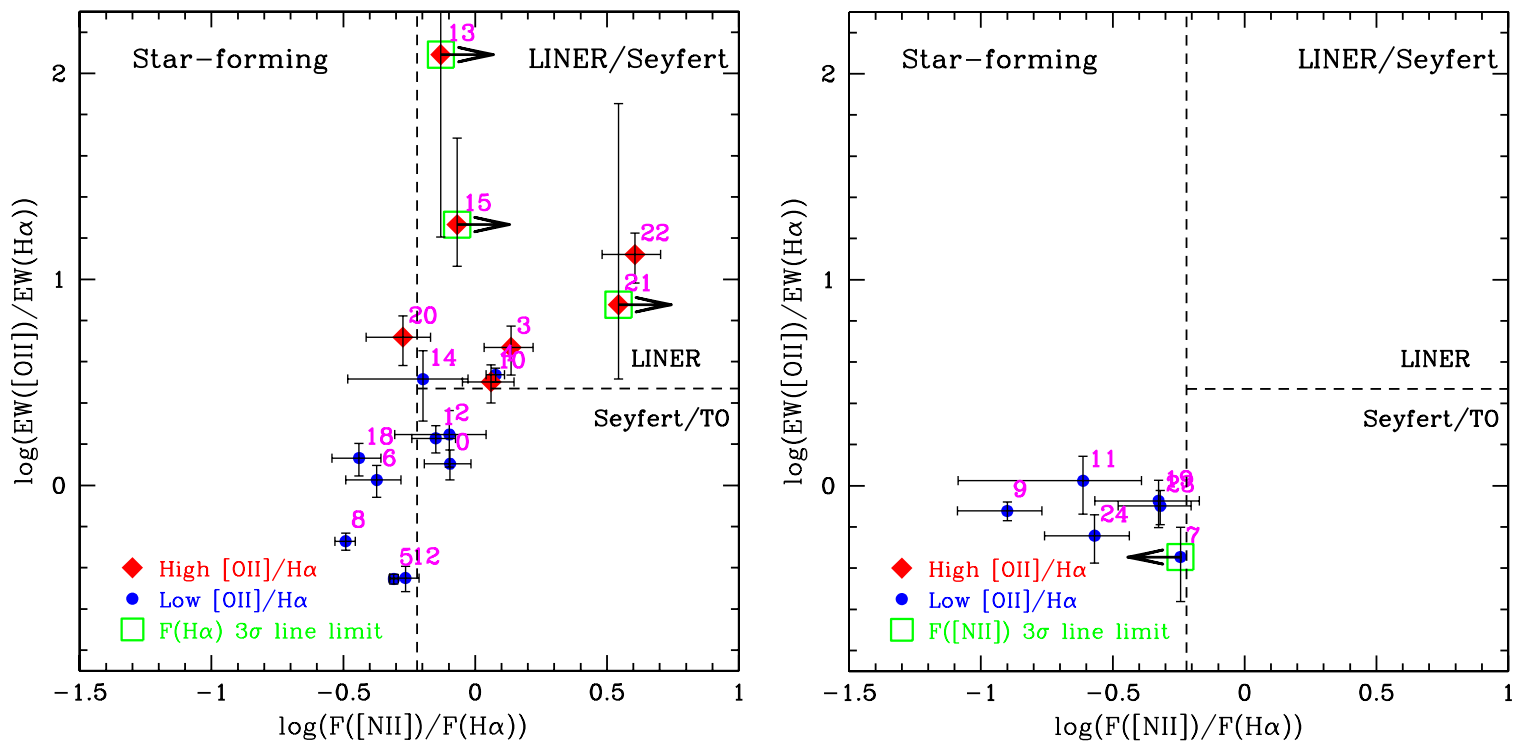

Figure 12. Left: ratio of $[\mathrm{O} \mathrm{II}]$ and $\mathrm{H} \alpha \mathrm{EWs}$ as a function of $F([\mathrm{~N} \mathrm{II}]) / F(\mathrm{H} \alpha)$ for 17 galaxies in the main sample that have at least $\mathrm{H} \alpha$ or $[\mathrm{N}$ II] detected at high $(>3 \sigma)$ significance. NIRSPEC targets in Cl1604 and RX J1821 are plotted. Galaxies with $3 \sigma$ upper limits on $F(\mathrm{H} \alpha)$ are plotted with arrows. All galaxies have $F([\mathrm{~N}$ II] $])$ detected at $>3 \sigma$. The vertical dashed line at $\log \left(F_{[\mathrm{N} \mathrm{II}] / \mathrm{H} \alpha}=-0.22\right)$ indicates our dividing line between star-forming and "LINER/Seyfert" galaxies, adopted from the criterion used in Kauffmann et al. (2003b). The horizontal dashed line at $\log (\mathrm{EW}([\mathrm{O}$ II $] / \mathrm{EW}(\mathrm{H} \alpha))=0.47$ denotes our rough dividing line between LINERs and Seyfert/TOs (see Section 5.2). Galaxy labels are identical to those in Figure 10. Eleven of the 17 galaxies plotted in the main sample are classified as LINER/Seyfert. Right: same plot for the six galaxies in the filler sample, all of which are classified as star forming. The galaxy with a $3 \sigma$ limit on $F([\mathrm{~N}$ II] $)$ is plotted with an arrow. All galaxies have $F(\mathrm{H} \alpha)$ detected at $>3 \sigma$. There is a general trend of increasing $\mathrm{EW}_{[\mathrm{O}} \mathrm{II}_{]} / \mathrm{H} \alpha$ with increasing $\left.\log \left(F_{[\mathrm{N}} \mathrm{II}\right] / \mathrm{H} \alpha\right)$.

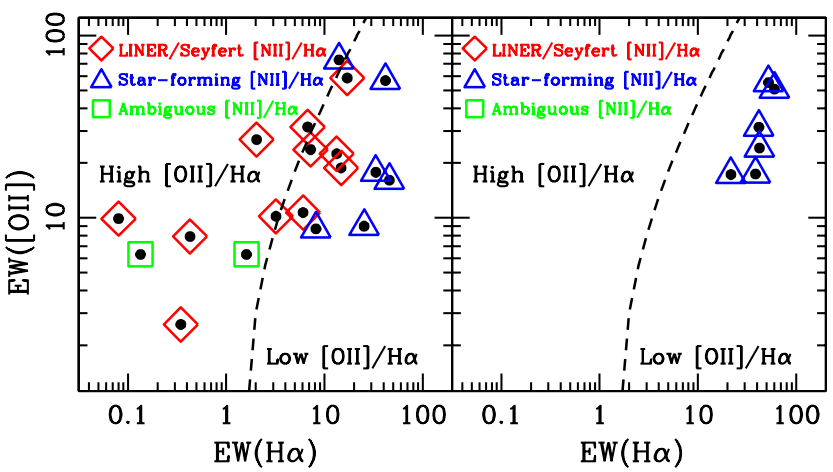

Figure 13. Same as in Figure 10, EW([O II]) vs. EW(H $\alpha$ ) for the 19 galaxies in the main sample (left) and the six galaxies in the filler sample (right). In this plot, the error bars and galaxy numbers are removed and replaced with the emission class of the galaxy. "Ambiguous" is used for galaxies that do not have detections in either $\mathrm{H} \alpha$ or [N II] and thus cannot be definitively classified as either star forming or LINER/Seyfert; however, it is likely that they belong to the latter class (see Section 5.3). The division between high $[\mathrm{O}$ II $] / \mathrm{H} \alpha$ and low $[\mathrm{O}$ II $] / \mathrm{H} \alpha$ is identical to that of Figure 10. A large fraction $(\sim 62 \%)$ of LINER/Seyfert and ambiguous galaxies are classified as high $[\mathrm{O} \mathrm{II}] / \mathrm{H} \alpha$. Galaxies classified as star forming are almost exclusively low $\left[\mathrm{O}_{\mathrm{II}}\right] / \mathrm{H} \alpha$. The one exception (galaxy 20) is likely a type 1.9 LINER/Seyfert (see Section 5.2). All filler galaxies are classified as low $[\mathrm{O}$ II] $/ \mathrm{H} \alpha$ and star forming.

star-forming population to redder colors and brighter magnitudes (see Figure 3). This result will be discussed further in Section 5.4.

Two galaxies (16 and 17), which we classify here as "ambiguous" galaxies, did not have significant detections of either $\mathrm{H} \alpha$ or $[\mathrm{NII}]$ despite having moderate $[\mathrm{O} \mathrm{II}]$ emission (EW([O II $]$ ) $\sim 7 \AA$ ) and, thus, are not plotted in Figure 12. These two galaxies share many of the same emission, color, and morphological properties as our LINER/Seyferts. The nature of the emission in these galaxies, which is likely due to a LINER or Seyfert, will be discussed further in Section 5.3.

Of the six galaxies comprising our filler (priority $\geqslant 2$ ) sample, all have $\log \left(F_{[\mathrm{NI}] / \mathrm{H} \alpha}\right) \leqslant-(0.22$, consistent with pure star formation. These results include galaxies with significant detections in either $\mathrm{H} \alpha$ or [N II] but no significant detection in the other line (plotted in Figure 12 with arrows). In all such cases, the $3 \sigma$ upper limit on the accompanying line was low enough to make a classification.

Though we cannot definitively distinguish between LINER, Seyfert, and TOs using $F_{[\mathrm{N} \text { II] } / \mathrm{H} \alpha}$ alone, the inclusion of $\mathrm{EW}([\mathrm{O} \mathrm{II}]) / \mathrm{EW}(\mathrm{H} \alpha)$ (hereafter $\left.\mathrm{EW}_{[\mathrm{O} \text { II }] / \mathrm{H} \alpha}\right)$ in our pseudo-BPT diagram allows for some distinction (i.e., Y06). LINERs exhibit typical $\mathrm{EW}_{[\mathrm{O} \text { II]/H } \alpha}$ values of $\sim 5$ while Seyferts and TOs have significantly smaller ratios of $\sim 1$. Though this ratio is somewhat sensitive to the metallicity of the host galaxy (see discussion in Section 5.4), the average $\mathrm{EW}_{[\mathrm{O} \text { II }] / \mathrm{H} \alpha}$ value of LINER/ Seyfert and ambiguous galaxies observed in this sample (median $\left.\mathrm{EW}_{[\mathrm{O}} \mathrm{II}_{/ \mathrm{H} \alpha}=3.9\right)$ strongly suggests this population is dominated by LINERs. The horizontal dashed line at $\log \left(\mathrm{EW}_{[\mathrm{OII}] / \mathrm{H} \alpha}\right)$ $=0.47$ in the two panels of Figure 12 provides a rough dividing line between LINERs and Seyferts/TOs.

As a consequence of this result, there is a noticeable trend between the ratio of [O II] and $\mathrm{H} \alpha$ EWs and $\log \left(F_{[\mathrm{NI}] / \mathrm{H} \alpha}\right)$; galaxies generally exhibit higher $\mathrm{EW}_{\left[\mathrm{O}_{\mathrm{II}}\right] / \mathrm{H} \alpha}$ ratios at higher values of $\log \left(F_{[\mathrm{N} \text { II }] / \mathrm{H} \alpha}\right)$. Of the 11 galaxies classified as LINER/ Seyfert, six are high [O II]/H $\alpha$. The trend of increasing LINER/ Seyfert fraction with higher $\mathrm{EW}_{[\mathrm{O}}{ }_{\mathrm{II}] / \mathrm{H} \alpha}$ can be seen in Figure 13. This plot is identical to Figure 10 except that the emission class of each galaxy is now indicated. All galaxies classified as star forming with one exception (galaxy 20) are significantly separated from the low-high $\mathrm{EW}_{\left[{ }_{[\mathrm{II}}\right] / \mathrm{H} \alpha}$ dividing line. LINER/ Seyferts cover a larger range in this phase space, but almost all lie nearly on or to the left of dividing line. Tables 5 and 6 list the total number of galaxies in both the main and filler sample and how these samples break down as a function of EW ratios and emission classes.

The notable exception to the trend of increasing LINER/ Seyfert fraction with higher $\mathrm{EW}_{[\mathrm{O} \text { II] } / \mathrm{H} \alpha}$ is galaxy 20. This galaxy is classified by its $\log \left(F_{[\mathrm{NII} / \mathrm{H} \alpha}\right)$ ratio as star forming but exhibits 
Table 5

Overview and Classifications of NIRSPEC Targets

\begin{tabular}{lcc}
\hline \hline \multicolumn{1}{c}{ Subset } & Main Sample & Filler Sample \\
\hline Total number & 19 & 6 \\
Cl1604 members & 17 & 4 \\
RX J1821 members & 2 & 2 \\
High [O II]/H $\alpha$ & $9(47.4 \%)$ & 0 \\
Low [O II]/H $\alpha$ & $10(52.6 \%)$ & $6(100 \%)$ \\
LINER/Seyfert & $11(57.9 \%)$ & 0 \\
Ambiguous & $2(10.5 \%)$ & 0 \\
Star forming & $6(31.6 \%)$ & $6(100 \%)$ \\
\hline
\end{tabular}

high levels of [O II] relative to $\mathrm{H} \alpha$. Both the [O II] emission and $\mathrm{H} \alpha$ in this galaxy appear slightly broadened $\left(\sim 300 \mathrm{~km} \mathrm{~s}^{-1}\right)$, typical of a type 1.9 LINER/Seyfert. The broad line features likely skew the $\log \left(F_{[\mathrm{NII} / \mathrm{H} \alpha}\right)$ ratio to a lower value, making the flux ratio difficult to interpret. Accordingly, we exclude this galaxy from any further analysis that compares the global properties of the LINER/Seyfert and star-forming samples.

Since the two ambiguous galaxies likely belong to the LINER/Seyfert class (albeit at a lower luminosity), the purity of the sample to the left of the dividing line suggests that at least a sub-population of LINER/Seyfert galaxies can be selected in high-redshift samples using only relative measurements of the [O II] and $\mathrm{H} \alpha$ lines. This selection is important because it does not rely on the detection of the (usually) fainter [N II], [S II], or [O I] lines or on absolute spectrophotometry. Galaxies that are dominated by LINER emission (and not Seyfert or TOlike emission) can likely be selected in this manner without much loss in completeness or purity (see Figures 12 and 13). Low-[O II $] / \mathrm{H} \alpha$ galaxies with LINER/Seyfert emission ratios, primarily Seyferts and TOs, will not be separable from star-forming galaxies using such a cut and must be selected, in the absence of optical forbidden lines, using other methods (e.g., X-ray emission, IR colors, radio power).

\subsection{Color, Spatial, and Morphological Properties of the NIRSPEC Sample}

We now investigate the color, spatial, and morphology distribution of our NIRSPEC sample. As shown in Figures 14 and 15, the galaxies in our sample range from faint blue-cloud galaxies in filaments between clusters (e.g., galaxies 7, 9, and 11) to the very reddest, most luminous galaxies at the center of wellestablished clusters (e.g., galaxies 13, 15, 16, and 17). Table 7 lists the $r^{\prime} i^{\prime} z^{\prime} K_{s}$ magnitudes for all RX J1821 and Cl1604 galaxies observed with NIRSPEC, as well as the ACS magnitudes and morphologies for our Cl1604 sample.

At low redshift, the dominant emission mechanism in red galaxies with appreciable [O II] is considerably different than in blue galaxies (Y06). Galaxies with high levels of [O II] relative to $\mathrm{H} \alpha$ are almost exclusively red and classed as LINERs. Conversely, blue galaxies with low $\mathrm{EW}_{[\mathrm{OII}] / \mathrm{H} \alpha}$ are predominantly star-forming galaxies. While a non-negligible fraction of red galaxies have low $\mathrm{EW}_{[\mathrm{OI}] / \mathrm{H} \alpha}$, these galaxies show a different $F_{[\mathrm{NII}] / \mathrm{H} \alpha}$ distribution than their blue counterparts, typically being classed as Seyferts or TOs.

In order to differentiate between galaxies of different colors in our own data, we use simple color-magnitude cuts to separate red-sequence galaxies from bluer members in both structures. These color cuts were originally done in observed $i^{\prime}-z^{\prime}$, roughly equivalent at $z=0.88$ to $\operatorname{SDSS}^{0.1}\left(g^{\prime}-r^{\prime}\right)$ cuts used by Y06 to differentiate between red and blue galaxies. In the Cl1604 field, we define the red sequence in terms of the $F 606 W-F 814 W$
Table 6

Classifications of the NIRSPEC Main Sample

\begin{tabular}{lcc}
\hline \hline Emission Class & High $[\mathrm{O}$ II] $/ \mathrm{H} \alpha$ & Low [O II] $/ \mathrm{H} \alpha$ \\
\hline LINER/Seyfert & $6(67 \%)$ & $5(50 \%)$ \\
Ambiguous & $2(22 \%)$ & 0 \\
Star forming & $1(11 \%)^{\mathrm{a}}$ & $5(50 \%)$ \\
\hline
\end{tabular}

Note. ${ }^{\text {a }}$ The one high-[O II] $/ \mathrm{H} \alpha$ galaxy classified as star forming is likely a type 1.9 LINER/Seyfert, see Section 5.2.

colors rather than LFC $i^{\prime}-z^{\prime}$. The red sequence is defined using the observed colors of all confirmed members in the magnitude range $21 \leqslant F 814 \leqslant 23$ for $\mathrm{Cl} 1604$ and $20 \leqslant z^{\prime} \leqslant 22.5$ for RX J1821. For each field, we fit the observed color distribution of the cluster galaxies with a linear function and subtract the best-fit color-magnitude relationship from the observed color distribution defining a "residual" color. For the two structures, the best-fit relationships are

$$
\begin{gathered}
F 606 W-F 814 W=3.182-0.063 F 814 W \mathrm{Cl} 1604, \\
i^{\prime}-z^{\prime}=1.887-0.058 z^{\prime} \mathrm{RXJ} 1821 .
\end{gathered}
$$

Following standard methodology of defining the red sequence in low- to moderate redshift clusters (Gladders et al. 1998; Stott et al. 2009), we adopt the $3 \sigma$ scatter in the residual colors $(\sigma=0.06)$ as the extent of the red sequence in color space for RX J1821. In Cl1604, we adopt $2 \sigma$ for the width of the red sequence, as the rms of the colors is much larger $(\sigma=0.0907)$ due to the extent of the supercluster in redshift space. Of the 25 galaxies in our sample, $60 \%$ (15 of 25) lie on the red sequence of the two structures, increasing to $70 \%$ (13 of 19) in our main sample (see Table 8). Ten galaxies (six in the main sample and four in the filler sample) have colors that are blueward of the red sequence.

The high-[O II] $] / \mathrm{H} \alpha$ sample consists of 8 of the 13 redsequence galaxies in the main sample and only one galaxy in the blue cloud (galaxy 10), similar to the observed properties of high-[O II $] / \mathrm{H} \alpha$ emitters at low redshift. The low-[O II $] / \mathrm{H} \alpha$ population includes five of the six galaxies in the main sample that are blueward of the red sequence. This population has similar emission mechanisms to the blue-cloud low-[O II $] / \mathrm{H} \alpha$ galaxies at low redshift, as $80 \%$ of the blue low-[O II $] / \mathrm{H} \alpha$ galaxies in the main sample have $F_{[\mathrm{NI}] / \mathrm{H} \alpha}$ values consistent with star formation. The one low-[O II $] / \mathrm{H} \alpha$ blue-cloud galaxy in the main sample that is not consistent with pure star formation (galaxy 2) lies extremely close to the red sequence.

While blue low-[O II $] / \mathrm{H} \alpha$ galaxies are primarily star forming, four of the five red-sequence low-[O $\mathrm{II}] / \mathrm{H} \alpha$ galaxies are classified as LINER/Seyfert. However, while these galaxies are classified as LINER/Seyfert, their $F_{[\mathrm{N} \mathrm{II]} / \mathrm{H} \alpha}$ values lie at the low end of the LINER/Seyfert distribution. Of all galaxies classified as LINER/Seyfert where both [N II] and $\mathrm{H} \alpha$ are detected with high significance, those with low-[O II] $/ \mathrm{H} \alpha$ values on or near the red sequence comprise five of the six lowest $F_{[\mathrm{N} \mathrm{II]} / \mathrm{H} \alpha}$ values. As $F_{[\mathrm{NII}] / \mathrm{H} \alpha}$ decreases in LINER/Seyfert galaxies, the fractional contribution to the ionizing flux from the LINER/Seyfert component decreases monotonically (see Kewley et al. 2001 for a detailed discussion). Therefore, it is likely that low-[O II $] /$ $\mathrm{H} \alpha$ LINER/Seyfert galaxies have the least dominant LINER/ Seyfert components and probably contain some residual star formation. Figure 12 confirms this result, as more than half of the low-[O II]/H $\alpha$ LINER/Seyferts lie below the line differentiating 

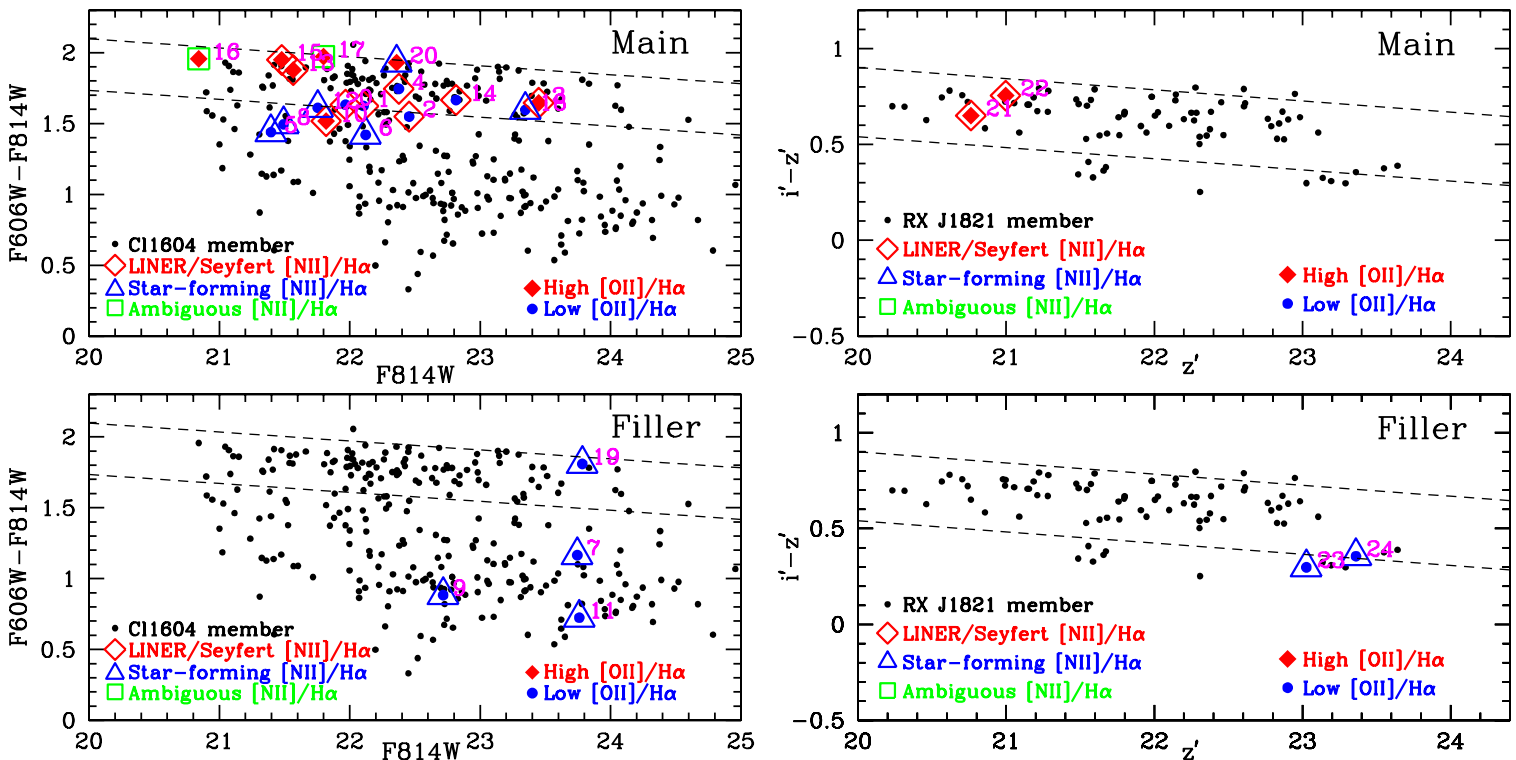

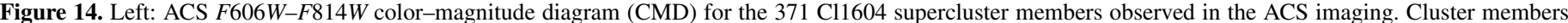

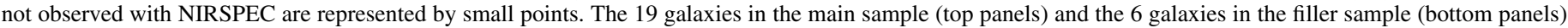

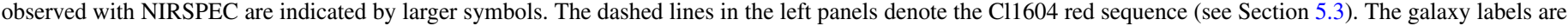

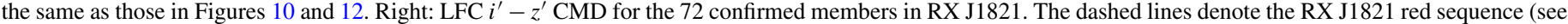

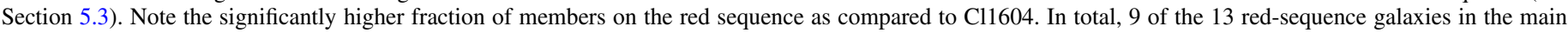

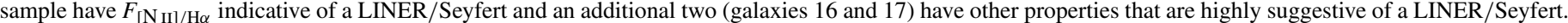

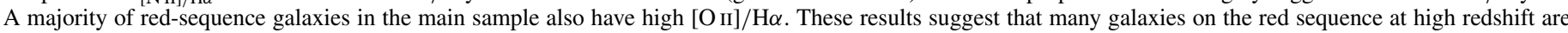

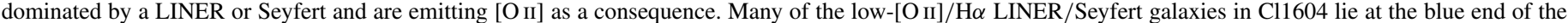
$\mathrm{Cl1604}$ red sequence, possibly suggesting that these galaxies are transitioning to the red sequence.

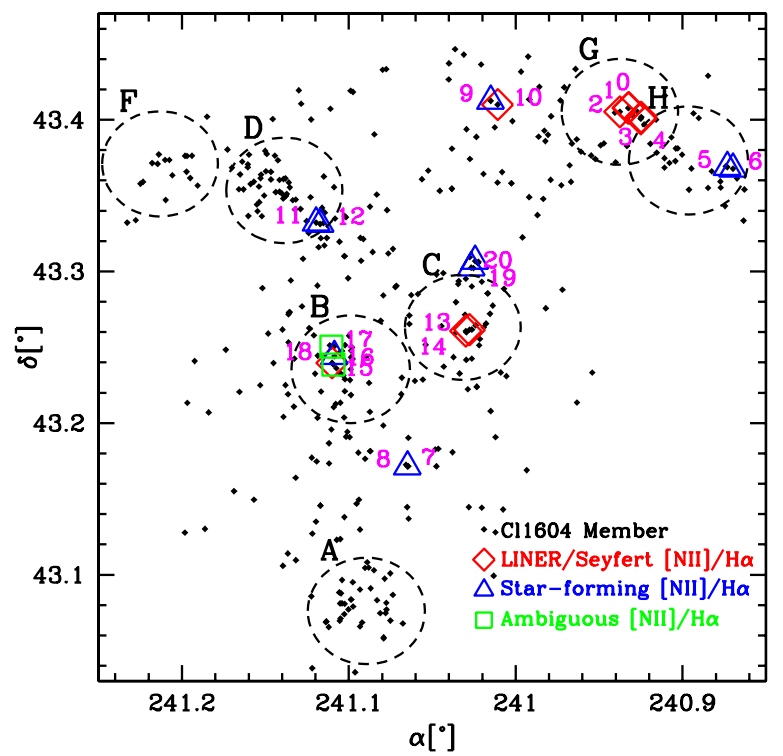

Figure 15. Spatial distribution of our Cl1604 targets plotted with the other 393 confirmed $\mathrm{Cl} 1604$ supercluster members. The letters for each constituent cluster or group are adopted from G08. The radius of each dashed line represents the angular extent of $1 h^{-1} \mathrm{Mpc}$ at the redshift of each group/cluster. Generally, the LINER/Seyfert and ambiguous galaxies lie at the centers of clusters and groups in the system. The structures that contain LINER/Seyfert galaxies extend over a large range in mass, from $313 \mathrm{~km} \mathrm{~s}^{-1}$ (cluster C) to $811 \mathrm{~km} \mathrm{~s}^{-1}$ (cluster B) (G08). Star-forming galaxies are generally found in the connecting filaments.

LINERs and Seyferts/TOs. The distributions of LINER/Seyfert and star-forming galaxies by color and EW ratio properties for the main sample are given in Table 9.

Two of the eight red-sequence high-[O II $] / \mathrm{H} \alpha$ galaxies were classified as ambiguous. These two galaxies (16 and 17) are similar to galaxies classified as LINER/Seyfert, both in their high levels of [O II] emission relative to their formal limits on $\mathrm{H} \alpha$ and in their color and spatial distributions. Indeed, almost all of the luminous red galaxies are classified as LINER/Seyfert and more than half have high levels of [O II] relative to $\mathrm{H} \alpha$, suggesting that these types of galaxies are typically dominated by a LINER or a Seyfert. Since five of the six red-sequence galaxies with high $[\mathrm{O} \mathrm{II}] / \mathrm{H} \alpha$ and well-defined $F_{[\mathrm{NI}] / \mathrm{H} \alpha}$ are classified as LINER/Seyfert (the one exception is galaxy 20, which is itself likely an AGN, see Section 5.2), ambiguous galaxies are also likely dominated by the same emission source. Based on these properties, we include these ambiguous galaxies in our sample of LINER/Seyfert galaxies.

The $F 606 W$ and $F 814 W$ images of each galaxy in the Cl1604 field were visually inspected by one of the authors (L.M.L.) in order to classify morphologies (galaxies in RX J1821 are excluded from this analysis due to the lack of ACS data). The morphologies of our sample show a spread in properties similar to that of their color and environments, ranging from irregular Sc galaxies (5 and 8) to isolated elliptical galaxies (3, 16, and 20). Table 7 lists the morphologies of all our targets. The nine galaxies classified as LINER/Seyfert in C11604, along with the two galaxies classified as ambiguous, are almost all earlytype galaxies, further supporting our claim that the ambiguous galaxies are similar to the LINER/Seyfert population. The exceptions are galaxies 0 and 1 , both morphologically classified as disk galaxies, although clearly disturbed by a merger or interaction. In total six galaxies are classified as star forming in the Cl1604 main sample. This population is dominated by late-type morphologies, with four of the six galaxies having morphologies consistent with either spirals or amorphous galaxies. The four galaxies in the Cl1604 filler sample, all classified as star forming, also had late-type morphologies. Automated measurements of galaxy compactness are similarly disparate between the two emission classes, as the LINER/Seyfert and ambiguous 
Table 7

Imaging Properties of the NIRSPEC Sample

\begin{tabular}{|c|c|c|c|c|c|c|c|c|c|c|c|c|}
\hline ID & Galaxy Number & $z$ & $\alpha_{2000}$ & $\delta_{2000}$ & $m_{F 606 W}$ & $m_{F 814 W}$ & $r^{\prime}$ & $i^{\prime}$ & $z^{\prime}$ & $K_{s}$ & Color $^{\mathrm{a}}$ & Morphology $\mathrm{y}^{\mathrm{b}}$ \\
\hline $\mathrm{J} 160344+432429$ & 0 & 0.9023 & 240.9322226 & 43.4079759 & 23.6011 & 21.9680 & 23.0289 & 21.6730 & 20.9049 &.$^{\mathrm{c}}$ & Red & S M I \\
\hline $\mathrm{J} 160344+432428$ & 1 & 0.9024 & 240.9325941 & 43.4077202 & 23.7290 & 22.1054 & 23.1568 & 21.8849 & 21.2576 & 20.5956 & Red & S C I \\
\hline $\mathrm{J} 160345+432419$ & 2 & 0.8803 & 240.9375426 & 43.4051985 & 24.0052 & 22.4560 & 23.7045 & 22.6779 & 22.0024 & 20.7284 & Blue & S0 \\
\hline $\mathrm{J} 160342+432406$ & 3 & 0.8986 & 240.9247136 & 43.4016956 & 25.0951 & 23.4488 & 23.9627 & 22.7284 & 22.2418 & 21.1584 & Blue & $\mathrm{E}$ \\
\hline $\mathrm{J} 160342+432403$ & 4 & 0.8959 & 240.9250684 & 43.4006981 & 24.1215 & 22.3766 & 23.4728 & 22.3332 & 21.5753 & 20.4692 & Red & E elongated \\
\hline $\mathrm{J} 160330+432208$ & 5 & 0.8983 & 240.8732075 & 43.3687725 & 22.8355 & 21.3960 & 22.4344 & 21.5230 & 20.8415 & 19.4092 & Blue & Sc I \\
\hline $\mathrm{J} 160329+432204$ & 6 & 0.9045 & 240.8697693 & 43.3676967 & 23.5419 & 22.1219 & 22.9297 & 21.8290 & 20.9793 & 20.5688 & Blue & Sa Ring \\
\hline $\mathrm{J} 160416+431021$ & 7 & 0.8990 & 241.0657080 & 43.1725670 & 24.9100 & 23.7452 & 24.9532 & 24.0943 & 22.4512 & $\ldots{ }^{c}$ & Blue & Amorphous \\
\hline $\mathrm{J} 160416+431017$ & 8 & 0.8999 & 241.0648269 & 43.1713681 & 22.9863 & 21.4923 & 22.4896 & 21.2475 & 20.4742 & 19.1758 & Blue & Sc I \\
\hline $\mathrm{J} 160404+432445$ & 9 & 0.9017 & 241.0150297 & 43.4124202 & 23.5993 & 22.7161 & 23.8893 & 22.8681 & 22.7997 & $\ldots{ }^{c}$ & Blue & $\mathrm{S}$ asymm $\mathrm{M}$ ? \\
\hline $\mathrm{J} 160403+432436$ & 10 & 0.9015 & 241.0108301 & 43.4099384 & 23.3368 & 21.8190 & 23.0297 & 21.9360 & 21.2114 & 19.7736 & Blue & $\mathrm{Sa} / \mathrm{S} 0$ \\
\hline $\mathrm{J} 160429+431956$ & 11 & 0.9185 & 241.1195420 & 43.3321920 & 24.4849 & 23.7607 & 25.1182 & 23.8742 & 23.8430 & $\ldots{ }^{c}$ & Blue & S0 peculiar I? \\
\hline $\mathrm{J} 160428+431953$ & 12 & 0.9198 & 241.1171400 & 43.3312750 & 23.3654 & 21.7545 & 23.1085 & 21.9486 & 21.2242 & 19.5014 & Blue & $\mathrm{SBb}$ \\
\hline $\mathrm{J} 160406+431542$ & 13 & 0.8674 & 241.0276264 & 43.2615940 & 23.4426 & 21.5654 & 22.5928 & 21.5389 & 20.7046 & 19.2990 & Red & S0 asymm \\
\hline $\mathrm{J} 160407+431539$ & 14 & 0.8676 & 241.0299022 & 43.2607188 & 24.4806 & 22.8149 & 23.9034 & 22.9086 & 22.1914 & 20.9843 & Red & elongated $\mathrm{E}$ \\
\hline $\mathrm{J} 160426+431423$ & 15 & 0.8676 & 241.1100259 & 43.2397136 & 23.4286 & 21.4794 & 21.9561 & 20.9147 & 19.9575 & 18.7911 & Red & E I? \\
\hline $\mathrm{J} 160426+431419$ & 16 & 0.8658 & 241.1092254 & 43.2386527 & 22.7983 & 20.8419 & 21.6547 & 20.3484 & 19.4208 & 18.3566 & Red & $\mathrm{E}$ \\
\hline $\mathrm{J} 160427+431501$ & 17 & 0.8601 & 241.1104629 & 43.2503720 & 23.7716 & 21.8000 & 22.8843 & 21.5115 & 20.5948 & 19.2840 & Red & E I? \\
\hline $\mathrm{J} 160426+431439$ & 18 & 0.8710 & 241.1086670 & 43.2441610 & 24.9447 & 23.3475 & 25.0073 & 23.4083 & 22.1090 & 21.3286 & Red & Amorphous? \\
\hline $\mathrm{J} 160406+431825$ & 19 & 0.9189 & 241.0243330 & 43.3068500 & 25.5927 & 23.7835 & 25.4611 & 23.9588 & 22.9134 & 21.2724 & Red & $\mathrm{S}$ disturbed \\
\hline $\mathrm{J} 160406+431809$ & 20 & 0.9195 & 241.0266087 & 43.3024702 & 24.2884 & 22.3585 & 22.7956 & 22.0227 & 21.1015 & 19.8396 & Red & $\mathrm{E}$ \\
\hline $\mathrm{J} 182110+682350$ & 21 & 0.7960 & 275.2922426 & 68.3971040 & $\ldots{ }^{\mathrm{d}}$ & $\ldots{ }^{\mathrm{d}}$ & 22.5616 & 21.4127 & 20.7620 & $\ldots \mathrm{e}^{\mathrm{e}}$ & Red & \\
\hline $\mathrm{J} 182108+682329$ & 22 & 0.8134 & 275.2819965 & 68.3941562 & $\ldots{ }^{d}$ & $\ldots{ }^{d}$ & 22.8643 & 21.7501 & 20.9960 & $\ldots^{\mathrm{e}}$ & Red & $\ldots{ }^{d}$ \\
\hline $\mathrm{J} 182121+682715$ & 23 & 0.8092 & 275.3361944 & 68.4540821 & $\ldots{ }^{d}$ & $\ldots{ }^{d}$ & 24.1828 & 23.3243 & 23.0264 & $\ldots{ }^{\mathrm{e}}$ & Blue & $\ldots{ }^{d}$ \\
\hline $\mathrm{J} 182123+682714$ & 24 & 0.8093 & 275.3460074 & 68.4539209 & $\ldots{ }^{d}$ & $\ldots{ }^{d}$ & 24.4237 & 23.7167 & 23.3609 & $\ldots^{\mathrm{e}}$ & Red & $\ldots{ }^{d}$ \\
\hline
\end{tabular}

Notes.

a As defined in Section 5.3.

b Done by visual inspection, M: merger, I: interaction, C: chaotic, S: spiral, asymm: asymmetric disk.

c Not detected in $K_{s}$.

d ACS data not available for RX J1821.

e $\mathrm{K}_{s}$ magnitudes not available for RX J1821.

Table 8

Properties of the NIRSPEC Main Sample by Color

\begin{tabular}{lcc}
\hline \hline \multicolumn{1}{c}{ Type } & Red & Blue \\
\hline Total & 13 & 6 \\
High $\left[\mathrm{O}_{\mathrm{II}}\right] / \mathrm{H} \alpha$ & $8(62 \%)$ & $1(17 \%)$ \\
Low $\left[\mathrm{O}_{\mathrm{II}}\right] / \mathrm{H} \alpha$ & $5(38 \%)$ & $5(83 \%)$ \\
LINER/Seyfert & $11(85 \%)^{\mathrm{a}}$ & $2(33 \%)$ \\
Star forming & $2(15 \%)^{\mathrm{b}}$ & $4(67 \%)$ \\
Early type & $8(73 \%)^{\mathrm{c}}$ & $2(33 \%)$ \\
Late type & $3(27 \%)^{\mathrm{c}}$ & $4(67 \%)$ \\
\hline
\end{tabular}

Notes.

a The two ambiguous galaxies are included here in the LINER/Seyfert category, see Section 5.3.

b One galaxy (20) classified as star forming is likely a type 1.9 LINER/Seyfert, see Section 5.2.

${ }^{\mathrm{c}}$ Only 11 of the 13 red-sequence galaxies in the main sample have morphological information.

galaxies are, on average, more compact than the star-forming galaxies at the $98 \% \mathrm{CL}$. Table 8 lists the distribution of EW ratios, emission classes, and morphological types as a function of color for the main sample.

\subsection{Understanding the Nature of [O II] Emission in LINER/Seyfert Galaxies}

While the high levels of [O II] relative to $\mathrm{H} \alpha$ correlate well with the presence of a LINER/Seyfert in a galaxy, it may still be the case that the [O II] emission in such galaxies is due to metallicity or extinction effects. In Figure 16, we plot
Table 9

Properties of the NIRSPEC Main Sample by Emission Class and Color

\begin{tabular}{|c|c|c|c|c|}
\hline & \multicolumn{2}{|c|}{ Red } & \multicolumn{2}{|c|}{ Blue } \\
\hline & \multicolumn{4}{|c|}{$\operatorname{High}\left[\mathrm{O}_{\text {II }}\right] / \mathrm{H} \alpha$ Low $\left[\mathrm{O}_{\text {II }}\right] / \mathrm{H} \alpha \operatorname{High}\left[\mathrm{O}_{\text {II }}\right] / \mathrm{H} \alpha$ Low $\left[\mathrm{O}_{\mathrm{II}}\right] / \mathrm{H} \alpha$} \\
\hline LINER/Seyfert & $7(54 \%)^{\mathrm{a}}$ & $4(30 \%)$ & $1(8 \%)$ & $1(8 \%)$ \\
\hline Star forming & $1(17 \%)^{b}$ & $1(17 \%)$ & 0 & $4(66 \%)$ \\
\hline
\end{tabular}

Notes.

a The two ambiguous galaxies are included here in the LINER/Seyfert category, see Section 5.3.

b One galaxy (20) classified as star forming is likely a type 1.9 LINER/Seyfert, Section 5.2.

the ratio of the extinction-corrected [O II] and $\mathrm{H} \alpha$ luminosities (hereafter $L_{[\mathrm{OII}] / \mathrm{H} \alpha}$ ) versus $F_{[\mathrm{NII}] / \mathrm{H} \alpha}$ for the $19 \mathrm{Cl} 1604$ targets with significant detections in [O II] and at least one of the two other spectral features $(\mathrm{H} \alpha$ or $[\mathrm{N}$ II] $)$. While there are significant exceptions (e.g., galaxies 9,10,11, and 20), there is a general trend of increasing [O II] luminosity relative to $\mathrm{H} \alpha$ for galaxies with higher levels of [N II] relative to $\mathrm{H} \alpha$. Excluding galaxy 20 (likely a type 1.9 LINER/Seyfert), a Spearman rank correlation coefficient test on our Cl1604 NIRSPEC sample results in a probability of positive correlation between the two ratios of $83 \%$, increasing to $>99.99 \%$ when only the 14 galaxies in the main sample are used. Line luminosities are extinction corrected using a constant value of $E(B-V)=0.3$ rather than individual extinction corrections based on the techniques discussed in Section 4.2.3 and Appendix B, as the overall trend (and Spearman rank coefficient) remains virtually unchanged 


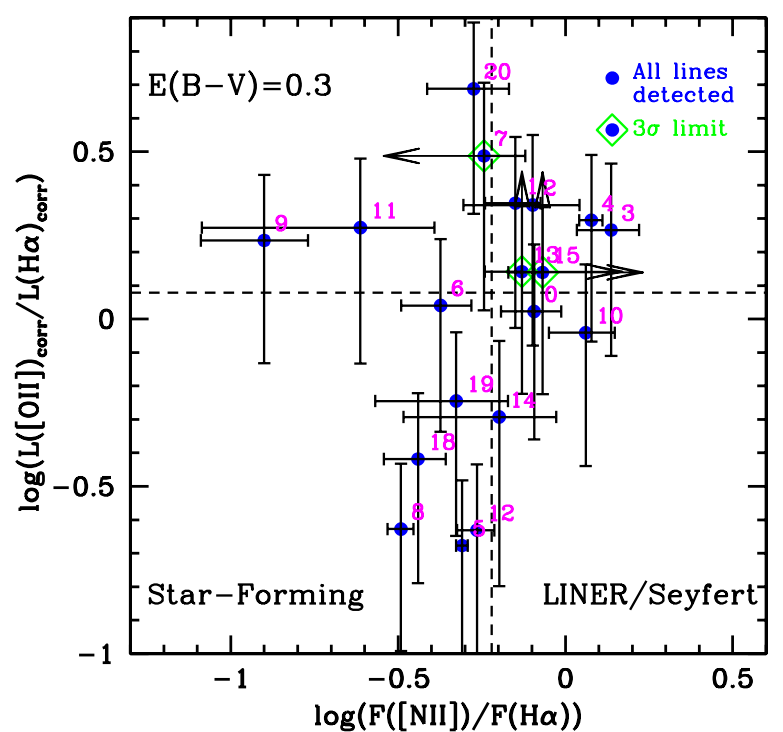

Figure 16. Logarithm of the ratio of extinction-corrected $L\left(\left[\mathrm{O}_{\mathrm{II}}\right]\right)$ and $L(\mathrm{H} \alpha)$ as a function of $\left.\log \left(F_{[\mathrm{N}} \mathrm{II}\right] / \mathrm{H} \alpha\right)$ for the $\mathrm{Cl} 1604$ galaxies in our sample. The extinction correction is performed using a constant $E(B-V)=0.3$ and the Calzetti et al. (2000) reddening law for all galaxies. Galaxies 16 and 17, which do not have significant detections in either $\mathrm{H} \alpha$ or $[\mathrm{N}$ II], are omitted from this plot. Galaxies with significant detections in either $\mathrm{H} \alpha$ or [N II], but with no detection in the accompanying line have $3 \sigma$ upper limits plotted as arrows. The vertical dashed line at $F_{[\mathrm{N} \mathrm{II]} / \mathrm{H} \alpha}=-0.22$. denotes our boundary between a star-forming and a LINER/Seyfert classification adapted from Kauffmann et al. (2003b). The horizontal dashed line $\left[\log \left(L_{\left[\mathrm{O}_{\amalg]} / \mathrm{H}_{\alpha}\right.}\right)=0.08\right]$ is the average extinction-corrected luminosity ratio for star-forming galaxies at low redshift (Kewley et al. 2004). Almost all of the galaxies classified as LINER/Seyfert lie above this line, while many of the star-forming galaxies lie below. This result suggests that the LINER/Seyfert component contributes appreciably to the [O II] emission. There is a general trend of increasing [O II] emission relative to $\mathrm{H} \alpha$ with increasing $F_{[\mathrm{NII}] / \mathrm{H} \alpha}$, with a few notable exceptions.

(A color version of this figure is available in the online journal.)

regardless of our choice of extinction correction. Thus, the observed excess [O II] emission in LINER/Seyfert galaxies is not likely due to dust effects.

Excluding galaxy 20 from the star-forming sample (since it is likely a type $1.9 \mathrm{LINER} /$ Seyfert), the mean $L_{[\mathrm{OII} / \mathrm{H} \alpha}$ ratio for star-forming galaxies is $1.04 \pm 0.27$, consistent with the average extinction-corrected $L_{[\mathrm{O} \text { II]/H } \alpha}$ of 1.2 (shown as a dashed line in Figure 16) found for the NFGS sample analyzed by Kewley et al. (2004). The lower limit on the average corrected $L_{[\mathrm{O} \text { II] } / \mathrm{H} \alpha}$ ratio for all galaxies classified as LINER/Seyferts is $1.51 \pm 0.28$, higher than both that of our star-forming galaxies and the lowredshift NFGS sample. This mean is a lower limit because several of the LINER/Seyfert galaxies in our sample have $3 \sigma$ upper limits on their $\mathrm{H} \alpha$ luminosities. Removing these galaxies from our sample increases the mean $L_{[\mathrm{O}} \mathrm{II} / \mathrm{H} \alpha$ for LINER/Seyfert galaxies, confirming the significance of this result.

This ratio, however, can be strongly affected by galaxy metallicity. The effect of increasing metallicity on this ratio for galaxies with $Z \gtrsim 0.6 Z_{\odot}$ is to decrease the intrinsic $\left.L_{[\mathrm{O}}{ }_{\mathrm{II}}\right] / \mathrm{H} \alpha$. The average metallicity of the low-redshift NFGS sample is just above solar $[\langle\log (\mathrm{O} / \mathrm{H})+12\rangle \sim 8.75]$, using the metallicity calibration of Kewley \& Dopita (2002). If our targets are, on average, less metal enriched than the NFGS sample, the high value of $L_{[\mathrm{OII}] / \mathrm{H} \alpha}$ for the LINER/Seyfert galaxies may be attributed to residual star formation in these galaxies rather than the LINER/Seyfert component.

At low redshift in cluster environments, galaxy metallicities range from $\sim 1 Z_{\odot}$ to $>5 Z_{\odot}$ over the stellar mass range of our sample $\left(\log \left(M_{\star}\right)=10^{10}-10^{11.5} M_{\odot} ; \mathrm{R}\right.$. R. Gal et al. 2010, in preparation). Because little evolution in the metallicity of cluster red-sequence galaxies occurs from $z \sim 1$ to the present day (Kauffmann \& Charlot 1998; Kodama et al. 1998), the mean metallicity in our sample is likely equal to or higher than the NFGS sample. We can test this empirically using our DEIMOS spectral data. While our spectral coverage does not allow us to observe $\mathrm{H} \beta$ or [O III] to check abundances directly through the standard $\mathrm{R}_{23}$ diagnostic, we are able to constrain the $F\left([\mathrm{Ne}\right.$ III] $\lambda 3869 \AA) / F\left(\left[\mathrm{O}_{\mathrm{II}}\right]\right)$ ratio (hereafter $F_{[\mathrm{Ne} \text { III } /[\mathrm{O} \text { II }]}$, which has also been shown to be sensitive to metal abundance (Nagao et al. 2006). In all galaxies in the main sample, the observed $F_{[\mathrm{Ne} \text { III]/[O II }]}$ or the formal $3 \sigma$ upper bound on this ratio is $<0.05$. Using the Nagao et al. (2006) relationship, this value corresponds to metallicities that are at least solar $[\log (\mathrm{O} / \mathrm{H})+12$ $=8.69]$ and potentially much higher. While the $F_{[\mathrm{Ne} \mathrm{III} /[\mathrm{O} \text { II }]}$ ratio is also sensitive to the presence of an AGN, the overall effect of the AGN is to increase this ratio, thereby decreasing the metallicity estimate. Since our formal limit already places the NIRSPEC sample at equivalent or higher metallicities relative to the NFGS sample, removing the AGN contribution would simply push this limit to higher metallicities. Thus, it is unlikely that the higher [O II] luminosity in LINER/Seyfert galaxies is due to metallicity effects. Having ruled out dust and metallicity effects as causing the increased [O II] to $\mathrm{H} \alpha$ ratio, the dominant source of the [O II] emission in these galaxies must come from the LINER or Seyfert component itself. Thus, the [O II] emission in such galaxies cannot be directly tied with the star formation activity unless the LINER or Seyfert contribution is carefully subtracted.

\subsection{Prevalence of LINER and Seyfert Activity in Cluster Galaxies}

As stated earlier, 11 out of the $19(58 \%)$ galaxies in the main sample have $F_{[\mathrm{NII} / \mathrm{H} \alpha}$ ratios consistent with at least some contribution from a LINER or Seyfert. If we include also the two ambiguous galaxies (16 and 17) that have LINER/Seyfertlike properties, $\sim 68 \%$ of galaxies in the main sample are not consistent with pure star formation. This fraction increases to $85 \%(11 / 13)$ for red-sequence galaxies in the main sample. For bluer galaxies, the fraction is much less; only $33 \%(2 / 6)$ of blue galaxies in the main sample have emission consistent with contributions from LINER/Seyfert sources.

The 19 galaxies that comprise our main sample do not represent a special sub-sample of the priority 1 galaxies. Priority 1 targets were selected for observation only on the basis of close proximity of another priority 1 (or other high priority) object, a bias that is unlikely to affect their overall properties relative to the main population (see Section 3.2.1). Furthermore, the objects selected in our main sample span a wide range of [O II] EWs and are distributed nearly over the entire color-magnitude range occupied by the whole priority 1 sample (compare Figures 3 and 14). Of the 108 priority 1 targets in $\mathrm{Cl1604,50 \%}$ have redsequence colors. In RX J1821, this percentage is much higher at $\sim 87 \%$, likely due to the larger fraction of red galaxies in that cluster. The difference in color properties between the two structures is significant since the fraction of priority 1 galaxies that are LINER/Seyfert in our main sample changes as a function of color (i.e., $85 \%$ of red priority 1 galaxies versus $33 \%$ of blue priority 1 galaxies).

Assuming the observed fractions of LINER/Seyfert in the main sample are representative of the whole priority 1 population, our results suggest that $\sim 20 \%$ of all cluster members 
at high redshift with $M_{\star} \gtrsim 10^{10}-10^{10.5} M_{\odot}$ (our rough stellar mass completeness limit, see Appendix B) contain a LINER/ Seyfert component that can be revealed by line ratios. Due to the conservative nature of our LINER/Seyfert selection (i.e., our preference for purity over completeness) this number is likely a lower limit for galaxies in this mass range, increasing by as much as a factor of 2 if $\mathrm{H} \beta$ and [O III] were available in our data. Specifically, if we make a cut identical to the one we have used in this paper on the low-redshift data of Kauffmann et al. (2003b), this results in a LINER/Seyfert sample that is only $\sim 50 \%$ complete. Conversely, if we were to probe less massive galaxies in the two structures the total fraction of structure members classified as LINER/Seyfert would likely decrease. This is due to the small fractional number $(\sim 10 \%)$ of galaxies with $M_{\star}<10^{10}$ at low redshift that contain LINER or Seyfert components (Kauffmann et al. 2003b).

It is remarkable that in both $\mathrm{Cl} 1604$ and $\mathrm{RX} \mathrm{J} 1821$, structures with significantly different populations, different DEIMOS selection functions, and different global spectral properties (see L09), the total fraction of priority 1 DEIMOS members is almost identical, 34.5\% (108/313) in Cl1604 and 31.5\% (23/73) in RX J1821. This fraction is, however, somewhat sensitive to the cluster sample used. If we instead cut both spectroscopic samples at our rough completeness limit of $i^{\prime} \sim 23$, the fraction of priority 1 galaxies in Cl1604 increases $(46.0 \%)$ while the fraction in RX J1821 remains roughly constant (30.6\%). Regardless of the sample used, the fraction of priority 1 galaxies in the two systems remains a significant fraction of the cluster galaxy population. Since a large fraction of priority 1 galaxies are likely LINER/Seyfert (i.e., $\sim 68 \%$ ), such galaxies seem to constitute a large fraction of the galaxy population in clusters at very different stages in their dynamical evolution. This result is consistent with observations of field and group galaxies at $z \sim 0.8$, in which LINERs are preferentially found in denser environments (Montero-Dorta et al. 2009). The similar fraction of LINER/Seyfert galaxies in Cl1604 and RX J1821 suggests that, whatever mechanism is powering their emission (i.e., either a LINER or Seyfert), this mechanism is long lived and active in cluster galaxies for much longer than the dynamical timescale of the cluster.

Similarities in the incidence of galaxies powered by LINER/ Seyfert activity can also be seen across cosmic time. At low redshift in both field and cluster environments, $\sim 40 \%$ of all galaxies on the red sequence exhibit appreciable [O II] emission, typically due to LINER or Seyfert activity. At high redshift, in the Cl1604 supercluster environment, 54\% of all galaxies on the red sequence (and a similar fraction in RX J1821) have appreciable levels of [O II] emission, most of which are also likely powered by the same phase of LINER/Seyfert emission observed at low redshift. The similarity in the fraction of priority 1 galaxies over a large range of environments and the similarity in the fractional number of red [O II] emitters across a wide redshift range suggests that this phenomenon is not enhanced or suppressed by the cluster environment and further reinforces the conclusion that this phase is long lived.

\subsection{Consequences for High-redshift Galaxy Surveys}

Having established that the LINER/Seyfert population constitutes a large fraction of galaxies at both high and low redshifts in both field and cluster environments, we now examine the consequences of our findings for large galaxy surveys at high redshift. For high-redshift surveys relying on [O II] emission as an SFR indicator, widespread LINER/Seyfert emission can

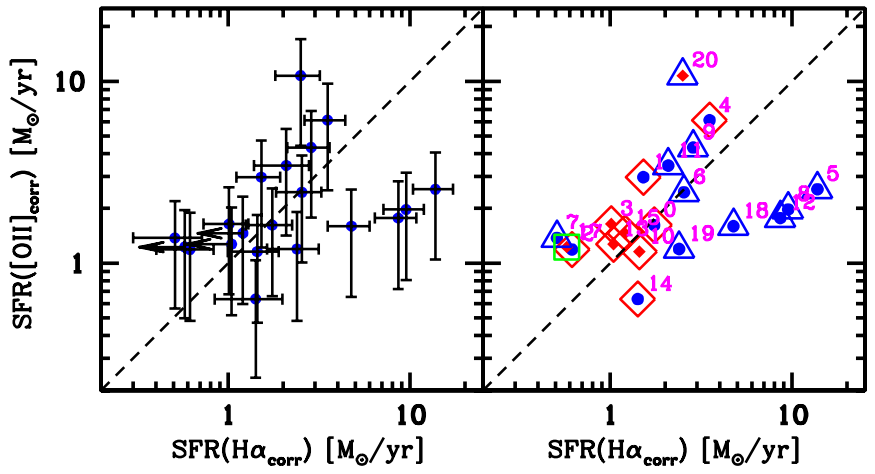

Figure 17. Comparison of star formation rates (SFRs) for our Cl604 sample as derived from extinction-corrected [O II] and $\mathrm{H} \alpha$ luminosities. The K98 SFR conversion is used. All galaxies are corrected for internal extinction using a constant $E(B-V)=0.3$ and the Calzetti et al. (2000) reddening law. The extinction at $\left[\mathrm{O}_{\mathrm{II}}\right]$ is corrected using the extinction at the wavelength of $\mathrm{H} \alpha$ due to the way the K98 [O II] SFR conversion was calibrated. Left: [O II] and H $\alpha$ SFRs with error bars. Galaxies with $3 \sigma$ upper limits for $L(\mathrm{H} \alpha)$ are plotted with horizontal arrows. The dashed line marks where the SFR from the two indicators is equal. Right: similar to the left panel, but with error bars replaced by the galaxy emission class, EW properties, and galaxy numbers. High-[O II] $/ \mathrm{H} \alpha$ galaxies are plotted as smaller filled diamonds and low-[O II $] / \mathrm{H} \alpha$ galaxies are plotted as smaller filled circles. Galaxies classified as LINER/Seyfert are plotted as large open diamonds, ambiguous galaxies are plotted as large open squares, and galaxies classified as star forming are plotted as large open triangles. There are two clear trends away from the unity (dashed) line. Galaxies that lie significantly to the right of line are all $24 \mu \mathrm{m}$ bright and are likely dust-reddened starbursts for which the effects of extinction are undercompensated. Galaxies to the left of the line, for which the [O II] SFR is higher than the H $\alpha$ SFR, are primarily LINER/Seyfert galaxies.

(A color version of this figure is available in the online journal.)

significantly bias results in several ways. The first and most obvious bias is introduced by incorrectly attributing [O II] emission to star formation processes rather than LINER/Seyfert emission, artificially inflating the measured global SFR or the star formation rate density (SFRD). A potentially more subtle bias comes when comparing the properties of galaxies of different spectral types in cluster or field studies as a function of local density or other environmentally sensitive parameters in order to constrain models of galaxy evolution. In Cl1604, for example, the average EW([O II]) for our LINER/Seyfert sample is $18 \AA$, high enough to be classified as starburst or star forming in any high-redshift survey (Balogh et al. 1999; Poggianti et al. 2006; Franzetti et al. 2007; Oemler et al. 2009). As the spatial distribution and fractions of various populations (most notably $\mathrm{K}+\mathrm{A}$ galaxies) are critical for many evolutionary studies in clusters, properly accounting for LINER/Seyferts is crucial. This is particularly important when comparing studies across a broad redshift range, as such quantities (e.g., SFRDs, fractional populations, etc.) derived at high redshift may be significantly biased relative to measurements made at low redshift where the $\mathrm{H} \alpha$ line (or another optical recombination line) is used as a star formation indicator.

To investigate the effect of the first bias on the calculated global SFR, we calculate the SFR of cluster galaxies using the relations of Kennicutt (1998, hereafter K98), due to their wide use as a conversion between the strength of nebular recombination lines and SFR. Figure 17 compares the SFR calculated using the K98 relations from the extinction-corrected $\mathrm{H} \alpha$ line and from the extinction-corrected [O II] using a constant $E(B-V)=0.3$ (the extinction correction to [O $\mathrm{II}]$ is made using the extinction at $\mathrm{H} \alpha$ due to the way that the K98 relations were calibrated). Two main deviations from the unity line are 
evident in Figure 17. The first is a population of galaxies at high$\mathrm{H} \alpha \mathrm{SFR} /$ low-[O II] SFR, which is likely due to undercorrected extinction as most of these galaxies are $24 \mu \mathrm{m}$ bright sources. The second is a population of galaxies with high-[O II] SFRs relative to their $\mathrm{H} \alpha \mathrm{SFRs}$, that are almost exclusively classified as LINER/Seyfert or ambiguous. The SFR as determined by the extinction-corrected [O II] luminosity in the LINER/Seyfert and ambiguous galaxies are on average $41 \%$ higher than those calculated using the $\mathrm{H} \alpha \mathrm{K} 98$ relation. This discrepancy is a lower limit, as 3 of the 10 galaxies used for this comparison have $\mathrm{H} \alpha \mathrm{SFRs}$ calculated from $3 \sigma$ upper limits. In contrast, the nine star-forming galaxies in this sample (excluding galaxy 20 from this analysis) have [O II] derived SFRs that are on average $8 \%$ lower than SFRs calculated from $\mathrm{H} \alpha$, consistent with no difference.

Since both $\mathrm{H} \alpha$ and [O II] are emitted by LINER/Seyferttype galaxies, using either the $\mathrm{H} \alpha$ or [O II] SFR conversion does not accurately reflect the star formation properties of these galaxies. Though some contribution to the $\mathrm{H} \alpha$ and [O II] fluxes likely come from $\mathrm{H}$ II regions in these galaxies, especially for TOs, the "contamination" of the emission lines by the LINER/ Seyfert component means that an emission-line derived SFR will always be an overestimate of the true value. While the SFR will be overestimated regardless of whether $\mathrm{H} \alpha$ or [O II] is used as a proxy, this analysis suggests that the problem becomes worse when one uses [O II] as an SFR indicator. While it has been suggested that [O II] be abandoned as an SFR indicator in cases when $\mathrm{H} \beta$ falls in the spectral window (Y06), corrections to the [O II] derived SFR proposed by Silverman et al. (2009) using the [O III] or X-ray luminosity when available may be useful in properly accounting for the LINER/Seyfert contribution and may be preferable in some cases.

Finally, we analyze the effect that this population of LINER/ Seyfert [O II] emitters has on the classification of post-starburst (i.e., $\mathrm{K}+\mathrm{A})$ galaxies. Typically, at high redshift $(z>0.3)$ a galaxy is classified as $\mathrm{K}+\mathrm{A}$ based on two criteria: (1) the presence of a strong A-star population evidenced by strong absorption in the hydrogen Balmer series (typically proxied by $\mathrm{H} \delta$ ) and (2) no active star formation proxied by the absence of [O II] emission. These $\mathrm{K}+\mathrm{A}$ galaxies may represent a crucial link in the transition of cluster and field galaxies from blue/ late-type to red/early-type galaxies. If we adopt one standard selection of post-starburst galaxies (e.g., EW([O II $]$ ) $<5 \AA$ and $\mathrm{EW}(\mathrm{H} \delta)<-5 \AA$; Balogh et al. 1999), 9.3\% (29/313) of all measurable Cl1604 spectra obtained with DEIMOS would be considered post-starburst, an intermediate number compared to the extreme ends of the distribution of $\mathrm{K}+\mathrm{A}$ fractions found in other high-redshift cluster populations (Dressler et al. 1999; Balogh et al. 1999).

While many galaxies classified as LINER/Seyfert may have some residual star formation, their optical emission profiles are dominated by the LINER/Seyfert component. Kauffmann et al. (2003b) estimated for the bright end $\left(L_{[\mathrm{O} \text { III }]}>3.83 \times 10^{41}\right.$ erg $\mathrm{s}^{-1}$ ) of such cases, the LINER/Seyfert component contributes, on average, $50 \%$ of the total [O II] luminosity. Thus, classification based on [O II] is not a sufficient criterion to rule out these galaxies as genuine post-starbursts. If we make the simple assumption that all galaxies classified as LINER/Seyfert have ceased forming stars and that the statistics of our main sample can be applied to the whole priority 1 population, the fraction of galaxies with recently truncated starburts $[\mathrm{EW}(\mathrm{H} \delta)<-5 \AA]$ in the DEIMOS Cl1604 sample increases to $18.8 \%(59 / 313)$. This result suggests that using traditional definitions of the $\mathrm{K}+\mathrm{A}$ classification severely undercounts galaxies that have recently ended their star formation activity, perhaps by as much as $\sim 50 \%$. This value is only a rough estimate due to the oversimplified nature of the assumption (especially true for TOs). A more thorough investigation of the star formation histories of galaxies with LINER/Seyfert-like properties using stellar synthesis modeling is necessary to fully quantify the effect of $\mathrm{H} \delta$-strong LINER/Seyfert galaxies on post-starburst selection.

Priority 1 galaxies, of which some fraction are LINER/ Seyfert "post-starburst," have a larger range of colors (i.e., both redder and bluer) and absolute magnitudes than populations of "traditional" K+A galaxies found in clusters (e.g., Poggianti et al. 1999; Dressler et al. 1999; see Figure 3). This disparity suggests that the LINER/Seyferts in our sample also represent a different class of post-starburst galaxy and may have different star formation histories and progenitors than their more traditional counterparts. The models of Poggianti et al. (1999), which attempted to identify the progenitors of traditional $\mathrm{K}+\mathrm{A}$ galaxies, relied heavily on the observed magnitude and color distribution of various spectral types. Including LINER/Seyfert poststarburst galaxies in such post-starburst samples would skew the overall color-magnitude distribution to redder colors and brighter magnitudes.

The relationship of LINER/Seyfert post-starburst galaxies to their traditional counterparts is not clear from these data. The fact that red priority 1 galaxies show a much higher fraction of LINER/Seyfert galaxies than their blue counterparts strongly suggests that these populations lie at different stages in their evolutionary history. The red priority 1 galaxies (of which $\sim 85 \%$ are LINER/Seyfert) also have Balmer absorption features that are, on average, weaker than their blue counterparts (of which $\sim 33 \%$ are LINER/Seyfert), suggesting that the time since the truncation of the star formation event is, on average, less for blue LINER/Seyfert galaxies. However, since we cannot discriminate between LINER/Seyfert emission and emission from a TO (the latter having ongoing star formation), whether or not the LINER/Seyfert mechanism is instrumental in the cessation or prevention of further star formation activity or whether it simply turns on after star formation has already been truncated by another process is not clear. This connection will be further investigated in a follow-up paper using multi-wavelength data to constrain the relative ages of the stellar populations in traditional post-starburst and LINER/Seyfert post-starburst galaxies. What is clear from our data, however, is that the LINER/Seyfert population in high-redshift clusters represents a substantial fraction of galaxies that are post-starburst or poststar-forming. Thus, in order to obtain a clear picture of galaxy evolution and to effectively link various populations in large surveys of galaxies it is necessary to account for contributions from LINER/Seyfert galaxies.

\section{CONCLUSIONS}

In this study, we have identified a population of [O II]emitting, absorption-line-dominated galaxies in high-redshift clusters that are primarily powered by LINER or Seyfert activity as evidenced by their optical and NIR spectroscopy. Of the 486 galaxies in the Cl1604 supercluster $(z \sim 0.9)$ and the X-ray-selected cluster RX J1821 $(z \sim 0.82)$ for which we have obtained optical spectroscopy, 25 galaxies were selected for follow-up NIRSPEC $J$-band spectra. These galaxies were primarily selected to be a representative sample of a population of cluster galaxies that have optical spectra that exhibits 
moderately strong [O II] emission with no other spectral indicators of current star formation, as well as strong absorption-line features indicative of a well-established older stellar population. Galaxies with these spectral properties (which we have termed "priority 1") make up a third of the population in both structures. The main results of this investigation are given below.

1 . We find that nearly half $(\sim 47 \%)$ of the [O II]-emitting, absorption-line-dominated galaxies in this study have high levels of [O II] emission relative to the amount of $\mathrm{H} \alpha$ emission.

2. Nearly all of galaxies with high levels of [O II] emission relative to $\mathrm{H} \alpha$ and a majority $(\sim 68 \%)$ of the targeted [O II]emitting, absorption-line-dominated galaxies have emission profiles dominated by a LINER or Seyfert component (referred to as LINER/Seyfert), primarily revealed by the flux ratio of $\mathrm{H} \alpha$ and $[\mathrm{N}$ II] $\lambda 6584$.

3. This LINER/Seyfert fraction has a strong dependence on color; $\sim 85 \%$ of targeted [O II]-emitting, absorptionline-dominated galaxies on the red sequence of the two structures have dominant LINER or Seyfert components as compared with only $33 \%$ of blue galaxies.

4. The bulk of our LINER/Seyfert population have observed $\mathrm{EW}([\mathrm{O} \mathrm{II}]) / \mathrm{EW}(\mathrm{H} \alpha)$ values significantly higher than unity, suggesting that a majority of these galaxies are powered by LINER and not Seyfert emission. The remainder are either powered by Seyfert emission or undergoing a transition phase in which both LINER/Seyfert and ongoing starforming activity is occurring.

5. In addition to being primarily red in color, galaxies powered by LINER or Seyfert emission are almost exclusively compact early-type galaxies, contrasting sharply with the latetype morphologies of the star-forming galaxies observed in our sample.

6. The lower limit to the average extinction-corrected $L([\mathrm{O} I \mathrm{II}]) / L(\mathrm{H} \alpha)$ in galaxies classified as LINER/Seyfert in our Cl1604 sample is $1.51 \pm 0.28$, higher than that of star-forming galaxies at low redshift (i.e., 1.2; Kewley et al. 2004 ) and that of the average star-forming galaxy observed in our sample $(1.04 \pm 0.27)$. We investigate various extinction schemes and metallicity differences in the samples and determine that the high levels of [O II] luminosity relative to $\mathrm{H} \alpha$ in LINER/Seyfert galaxies are not due to dust or metallicity effects and are rather the result of emission from the LINER/Seyfert itself.

From the statistical properties of this sample, we use the color distribution and prevalence of [O II]-emitting, absorptionline-dominated galaxies in our entire Cl1604 and RX J1821 DEIMOS database to determine the fraction of cluster galaxies at high redshift that contain a LINER/Seyfert component. For galaxies with stellar masses equal to or greater than our stellar mass limit (roughly $M_{\star}=10^{10}-10^{10.5} M_{\odot}$ ), we estimate that $>20 \%$ of galaxies in these structures contain a LINER/ Seyfert component. Additionally, the fraction of galaxies on the red sequence that have appreciable [O II] emission (most of which is likely due to a LINER or Seyfert) is $\sim 54 \%$ in both structures, similar to the fraction observed in red SDSS galaxies at low redshift. Since these two systems are in significantly different dynamical stages, these results imply that whatever mechanism is powering the emission in these galaxies is active for much longer than the dynamical timescale of the clusters and is not sensitive to the global environment in which a galaxy resides.
We have established that a large fraction of high-redshift galaxies, especially those on the red sequence, have [O II] emission directly resulting from a process unrelated to star formation. This result has significant consequences for surveys of high-redshift galaxies that use [O II] as a star formation indicator. The global SFR as calculated from the extinctioncorrected [O II] line luminosity for the LINER/Seyfert galaxies is significantly higher than the same quantity derived from the $\mathrm{H} \alpha$ feature, itself an overestimate of the actual SFR (due to $\mathrm{H} \alpha$ flux originating from the LINER/Seyfert component). We conclude that high-redshift galaxy surveys that rely on [O II] as an SFR indicator will be non-negligibly biased by LINER/ Seyfert activity. While other recombination lines (e.g., $\mathrm{H} \alpha, \mathrm{H} \beta$ ) provide better estimates of the instantaneous SFR than [O II] when observable, the problem of residual LINER/Seyfert flux still remains.

We also investigate the effect of the LINER/Seyfert population on the selection of transititory "post-starburst" galaxies, a population that is of considerable interest for many cluster and field evolutionary studies. We find that including $\mathrm{H} \delta$ strong LINER/Seyfert galaxies increases the percentage of post-starburst galaxies in the two structures to $18.8 \%$, more than double the $9.3 \%$ obtained using traditional selection methods. While some LINER/Seyfert galaxies likely still have some residual star formation, the requirement that [O II] be absent for a galaxy to be classified as post-starburst is too conservative and will result in a post-starburst sample that is severely incomplete. Due to the prevalence of LINER/Seyfert activity across a large range of environments at both high and low redshifts, we conclude that LINER/Seyferts must be carefully accounted for when interpreting post-starburst populations in the context of galaxy evolution.

We thank Jeff Newman and Michael Cooper for guidance with the spec $2 d$ reduction pipeline and for the many useful suggestions and modifications necessary to reduce our DEIMOS data. We thank Nick Konidaris for help with DEIMOS flux calibration and for useful discussions on equivalent width measurements in DEIMOS data. We also thank Chris Fassnacht for providing his notes on NIRSPEC observations and the Keck II support astronomers for their help with the observations. B.C.L. thanks Gary Creason for his patience and guidance. This material is based upon work supported by the National Aeronautics and Space Administration under Award NNG05GC34G for the Long Term Space Astrophysics Program. The spectrographic data presented herein were obtained at the W.M. Keck Observatory, which is operated as a scientific partnership among the California Institute of Technology, the University of California, and the National Aeronautics and Space Administration. The Observatory was made possible by the generous financial support of the W.M. Keck Foundation. We thank the indigenous Hawaiian community for allowing us to be guests on their sacred mountain; we are most fortunate to be able to conduct observations from this site.

\section{APPENDIX A}

\section{EQUIVALENT WIDTH MEASUREMENTS}

In order to measure the rest-frame $\mathrm{EW}$, the wavelength values of each observed-frame spectrum are divided by $(1+z)$. In all cases, the DEIMOS/LRIS redshift is used. In most cases, there were no significant differences between the NIRSPEC 
and DEIMOS redshifts, and the maximal offsets of $\Delta z=$ $0.0005-0.002\left(\Delta v \approx 100-300 \mathrm{~km} \mathrm{~s}^{-1}\right)$ have little effect on the EW measurement.

Line-fitting EW measurements were performed for all DEIMOS spectra, as our entire NIRSPEC sample had [O II] lines detected at a significance of greater than $3 \sigma$. For these spectra, we fit a double Gaussian model plus a linear continuum to the $3726 \AA$ and $3729 \AA$ [O II] doublet, which is typically resolved by the DEIMOS $12001 \mathrm{~mm}^{-1}$ grating. In cases where the [O II] doublet was not resolved, a double Gaussian model was still used. As these measurements were done in the rest frame, the two Gaussians were fixed to a separation of $2.8 \AA$. Thus, the model contained seven free parameters, two to characterize the linear continuum, four to characterize the FWHM and amplitude of each Gaussian, and a single parameter defining the mean wavelength of the blueward Gaussian.

For NIRSPEC data, we fit only those spectra where both $\mathrm{H} \alpha$ and $\left[\mathrm{N}_{\mathrm{II}}\right]$ were detected at greater than $3 \sigma$. Again, we use a seven parameter double Gaussian plus linear continuum to fit $\mathrm{H} \alpha$ and $\left[\mathrm{N}_{\mathrm{II}}\right]$, with a fixed separation of $20.6 \AA$. As noted in the text (see Section 4.1), adding a third Gaussian to account for the blueward [N II] $\lambda 6548$ feature had a negligible effect on the EW measurements. In all cases where fitting was used to determine EWs, errors were estimated from the covariance matrix of the fit.

Bandpass measurements were performed by defining two "continuum" bandpasses, slightly blueward and redward of the spectral feature, which are used to estimate the stellar continuum across the emission feature. An additional "feature" bandpass is defined to encompass the spectral line. A $\chi^{2}$ minimization to the linear continuum terms was performed over the two continuum bandpasses. Any pixels with large variance values (typically from bright sky features) were removed from the continuum bandpasses. We do not remove similar pixels in the feature bandpass. The EW is defined as

$$
\operatorname{EW}(\AA)=\sum_{i=0}^{n} \frac{F_{i}-C_{i}}{C_{i}} \lambda_{r, i},
$$

where $F_{i}$ is the flux in the $i$ th pixel in the feature bandpass, $C_{i}$ is the continuum flux in the $i$ th pixel over the same bandpass, and $\Delta \lambda_{r, i}$ is the rest-frame pixel scale of the spectrum (in $\AA$ pixel ${ }^{-1}$ ). Errors in the EW were derived using a combination of Poisson errors on the spectral feature and the covariance matrix of the linear continuum fit and are given by (Bohlin et al. 1983):

$$
\sigma_{\mathrm{EW}}(\AA)=\sqrt{\left(\sum_{i=0}^{n} \frac{\sigma_{F, i} \lambda_{i}}{C_{i}}\right)^{2}+\left(\sigma_{C} \sum_{i=0}^{n} \frac{F_{i} \lambda_{i}}{C_{i}^{2}}\right)^{2}} .
$$

Bandpasses were initially chosen to be "standard," using the bandpasses of Fisher et al. (1998) for the [O II] feature (blue continuum: [3696.3, 3716.3], red continuum: [3738.3, 3758.3], feature bandpass: [3716.3, 3738.3]) and the bandpasses of Y06 for the $\mathrm{H} \alpha$ and $[\mathrm{N} \mathrm{II}]$ features (for both features, blue continuum: [6483.0, 6513.0], red continuum: [6623.0,6653]; H $\alpha$ feature bandpass: [6554.6, 6574.6], [N $\mathrm{NI}$ ] feature bandpass: [6575.3, 6595.3]). These bandpasses were then modified by eye for each galaxy spectrum to avoid poorly subtracted airglow lines and to avoid "contaminate" features near the spectral lines of interest (primarily higher order Balmer lines when measuring [O II]).

For high $\mathrm{S} / \mathrm{N}$ spectral features, line-fitting techniques generally gave more accurate values and smaller errors for the EW, as noise in the data has a relatively small effect on the overall fit (Goto et al. 2003; Y06; Tremonti et al. 2004). For lower $\mathrm{S} / \mathrm{N}$ lines bandpass measurements generally led to more accurate results (Goto et al. 2003; Y06). Many of the spectral features that we are measuring (especially in the NIRSPEC data) have pixel $\mathrm{S} / \mathrm{N} \lesssim 5$, and these data can be severely affected by overor undersubtracted skylines. Therefore, we chose the EW measurements derived from bandpass techniques for a majority of EWs. In all cases where measurements of the [O II], $\mathrm{H} \alpha$, and $[\mathrm{N} \mathrm{II}]$ features were made using both techniques, the two methods agreed within the errors for high $\mathrm{S} / \mathrm{N}$ features. Only at low $\mathrm{S} / \mathrm{N}$, when the line-fitting technique began to fail, did the EW measurement differ appreciably between the two methods.

\section{APPENDIX B \\ EXTINCTION CORRECTION METHODS AND STELLAR MASSES}

The problem of extinction correction in this data set is complicated by the nature of our sample. Many conventional correction methods, such as those mentioned in section Section 4.2.3, are made assuming the dominant contribution to the recombination lines comes from HII regions rather than LINERs or Seyferts. As many of the galaxies in our sample contain either dominant LINER/Seyfert emission or a linear combination of LINER/Seyfert and star formation activity, assumptions such as a mean Balmer decrement or average observed [O II] $/ \mathrm{H} \alpha$ ratios are not necessarily valid for our sample. While we adopt a constant extinction value of $E(B-V)=0.3$ for our data (see Section 4.2.3), we report here on the three methods that were used to constrain our choice of $E(B-V)=0.3$ and to justify its use.

Extinction estimates from the $24 \mu \mathrm{m}$ data were made by comparing the SFR calculated through a linear combination of the observed $\mathrm{H} \alpha$ and $24 \mu \mathrm{m}$ luminosities using the formula of Calzetti et al. (2007). This value is compared with the value of the SFR measured using $\mathrm{H} \alpha$ alone. $K$-corrections from the observed MIPS luminosity to the rest-frame $24 \mu \mathrm{m}$ were derived using the templates of Chary \& Elbaz (2001). For the six galaxies with $24 \mu \mathrm{m}$ detections in the Cl1604 NIRSPEC sample, the $E(B-V)$ values range from $E(B-V)=0.15$ to 0.71 , with a mean of $0.32 \pm 0.09$.

The second method is based on absolute $B$-band magnitudes, which are estimated by $K$-correcting the observed $i^{\prime}$ magnitude (nearly identical to the Johnson $B$ band at the supercluster redshift) of each Cl1604 NIRSPEC target. Extinction values were generated using the Argence \& Lamareille (2009) adaptation of the best-fit $M_{B}-E(B-V)$ relationship of Moustakas et al. (2006). The derived $E(B-V)$ values range from 0 to 0.66 , with a mean of $0.29 \pm 0.07$ for the 20 galaxies in the $\mathrm{Cl} 1604$ NIRSPEC sample for which $K$-corrections could be performed.

The third estimate of extinction was derived from synthetic stellar template fits to the optical/IR SED using the Le PHARE ${ }^{7}$ (Arnouts \& Ilbert) codes with the single-burst stellar population models of Bruzual \& Charlot (2003). Using the redshift as a prior, the Le PHARE code provides an estimate of the stellar mass, stellar age, extinction, metallicity, and $\tau$ (the $e$-folding time of a single star formation event) for each galaxy. The stellar mass of C11604 members range from $M_{\star}=$ $10^{9} M_{\odot}$ to $10^{11.5} M_{\odot}$ (the NIRSPEC targets range from $M_{\star}=$ $10^{10}$ to $10^{11.5} M_{\odot}$ ). Our completeness limit, roughly proxied by the turnover in the supercluster galaxy mass function,

\footnotetext{
http://www.oamp.fr/people/arnouts/LE_PHARE.html
} 
corresponds to $M_{\star}=10^{10}-10^{10.5} M_{\odot}$. Extinction values were only used for galaxies that were cleanly detected in $r^{\prime} i^{\prime} z^{\prime} K_{s}$ and at least the first two IRAC channels (3.6 and $4.5 \mu \mathrm{m}$ ). This criterion is necessary as strong degeneracies exist between extinction and the other estimated parameters (e.g., metallicity and age) that are difficult to break without detections in the first two IRAC channels. The extinction values estimated for the 17 Cl1604 NIRSPEC targets that were detected in all six bands range from $E(B-V)=0$ to 0.4 , with a mean of $0.24 \pm 0.02$.

\section{REFERENCES}

Argence, B., \& Lamareille, F. 2009, A\&A, 495, 759

Baldwin, J. A., Phillips, M. M., \& Terlevich, R. 1981, PASP, 93, 5

Balogh, M. L., Morris, S. L., Yee, H. K. C., Carlberg, R. G., \& Ellingson, E. 1997, ApJ, 488, L75

Balogh, M. L., Morris, S. L., Yee, H. K. C., Carlberg, R. G., \& Ellingson, E. 1999, ApJ, 527, 54

Binette, L., Magris, C. G., Stasińska, G., \& Bruzual, A. G. 1994, A\&A, 292, 13

Bohlin, R. C., Jenkins, E. B., Spitzer, L., Jr., York, D. G., Hill, J. K., Savage, B. D., \& Snow, T. P., Jr. 1983, ApJS, 51, 277

Bruzual, G., \& Charlot, S. 2003, MNRAS, 344, 1000

Buat, V., et al. 2005, ApJ, 619, L51

Calzetti, D. 2001, PASP, 113, 1449

Calzetti, D., Armus, L., Bohlin, R. C., Kinney, A. L., Koornneef, J., \& StorchiBergmann, T. 2000, ApJ, 533, 682

Calzetti, D., Kinney, A. L., \& Storchi-Bergmann, T. 1994, ApJ, 429, 582

Calzetti, D., et al. 2007, ApJ, 666, 870

Caputi, K. I., et al. 2008, ApJ, 680, 939

Chary, R., \& Elbaz, D. 2001, ApJ, 556, 562

Colina, L., Bohlin, R. C., \& Castelli, F. 1996, Absolute Flux Calibration Spectrum of Vega (STScI Rep. OSG-CAL-96-01; Baltimore, MD: STScI)

Conroy, C. 2010, MNRAS, 404, 247

Cooper, M. C., et al. 2006, MNRAS, 370, 198

Couch, W. J., Ellis, R. S., Sharples, R. M., \& Smail, I. 1994, ApJ, 430, 121

Davis, M., et al. 2003, Proc. SPIE, 4834, 161

Dopita, M. A., \& Sutherland, R. S. 1995, ApJ, 455, 468

Dressler, A., \& Gunn, J. E. 1983, ApJ, 270, 7

Dressler, A., \& Gunn, J. E. 1988, in Proc. IAU Symp. 130, Large Scale Structures of the Universe, ed. J. Audouze, M.-C. Pelleton, \& S. Szalay (Dordrecht: Kluwer), 311

Dressler, A., \& Gunn, J. E. 1992, ApJS, 78, 1

Dressler, A., Oemler, A. J., Poggianti, B. M., Smail, I., Trager, S., Shectman, S. A., Couch, W. J., \& Ellis, R. S. 2004, ApJ, 617, 867

Dressler, A., Rigby, J., Oemler, A., Fritz, J., Poggianti, B. M., Rieke, G., \& Bai, L. 2009, ApJ, 693, 140

Dressler, A., Smail, I., Poggianti, B. M., Butcher, H., Couch, W. J., Ellis, R. S., \& Oemler, A. J. 1999, ApJS, 122, 51

Dressler, A., Thompson, I. B., \& Shectman, S. A. 1985, ApJ, 288, 481

Dressler, A., et al. 1997, ApJ, 490, 577

Erb, D. K., Shapley, A. E., Steidel, C. C., Pettini, M., Adelberger, K. L., Hunt, M. P., Moorwood, A. F. M., \& Cuby, J.-G. 2003, ApJ, 591, 101

Faber, S. M., et al. 2003, Proc. SPIE, 4841, 1657

Ferland, G. J., \& Netzer, H. 1983, ApJ, 264, 105

Filippenko, A. V. 2003, in ASP Conf. Ser. 290, Active Galactic Nuclei: From Central Engine to Host Galaxy, ed. S. Collin, F. Combes, \& I. Shlosman (San Francisco, CA: ASP), 369

Filippenko, A. V., \& Halpern, J. P. 1984, ApJ, 285, 458

Filippenko, A. V., \& Terlevich, R. 1992, ApJ, 397, L79

Fisher, D., Fabricant, D., Franx, M., \& van Dokkum, P. 1998, ApJ, 498, 195

Franzetti, P., et al. 2007, A\&A, 465, 711

Fukugita, M., Ichikawa, T., Gunn, J. E., Doi, M., Shimasaku, K., \& Schneider, D. P. 1996, AJ, 111, 1748

Gal, R. R., Lemaux, B. C., Lubin, L. M., Kocevksi, D., \& Squires, G. K. 2008, ApJ, 684, 933 (G08)

Gal, R. R., \& Lubin, L. M. 2004, ApJ, 607, L1

Gioia, I. M., Henry, J. P., Mullis, C. R., Böhringer, H., Briel, U. G., Voges, W., \& Huchra, J. P. 2003, ApJS, 149, 29

Gioia, I. M., Wolter, A., Mullis, C. R., Henry, J. P., Böhringer, H., \& Briel, U. G. 2004, A\&A, 428, 867

Gladders, M. D., Lopez-Cruz, O., Yee, H. K. C., \& Kodama, T. 1998, ApJ, 501, 571

Gómez, P. L., et al. 2003, ApJ, 584, 210

Goto, T., et al. 2003, PASJ, 55, 771
Hashimoto, Y., Oemler, A. J., Lin, H., \& Tucker, D. L. 1998, ApJ, 499, 589

Heckman, T. M. 1980, A\&A, 87, 152

Heckman, T. M. 1981, ApJ, 250, L59

Heckman, T. M., Baum, S. A., van Breugel, W. J. M., \& McCarthy, P. 1989, ApJ, 338, 48

Henry, J. P., Gioia, I. M., Mullis, C. R., Voges, W., Briel, U. G., Böhringer, H., \& Huchra, J. P. 2001, ApJ, 553, L109

Ho, L. C., Filippenko, A. V., \& Sargent, W. L. W. 1993, ApJ, 417, 63

Horne, K. 1986, PASP, 98, 609

Jansen, R. A. 2000, PhD thesis, Kapteyn Astronomical Institute

Jansen, R. A., Franx, M., \& Fabricant, D. 2001, ApJ, 551, 825

Kaufman, M., Bash, F. N., Kennicutt, R. C., Jr., \& Hodge, P. W. 1987, ApJ, 319, 61

Kauffmann, G., \& Charlot, S. 1998, MNRAS, 294, 705

Kauffmann, G., et al. 2003a, MNRAS, 341, 33

Kauffmann, G., et al. 2003b, MNRAS, 346, 1055

Kennicutt, R. C., Jr. 1983, ApJ, 272, 54

Kennicutt, R. C., Jr. 1998, ARA\&A, 36, 189

Kewley, L. J., Dopita, M. A., Sutherland, R. S., Heisler, C. A., \& Trevena, J. 2001, ApJ, 556, 121

Kewley, L. J., \& Dopita, M. A. 2002, ApJS, 142, 35

Kewley, L. J., \& Ellison, S. L. 2008, ApJ, 681, 1183

Kewley, L. J., Geller, M. J., \& Barton, E. J. 2006, AJ, 131, 2004

Kewley, L. J., Geller, M. J., \& Jansen, R. A. 2004, AJ, 127, 2002

Kewley, L. J., Geller, M. J., Jansen, R. A., \& Dopita, M. A. 2002, AJ, 124 3135

Kocevski, D. D., Lubin, L. M., Gal, R., Lemaux, B. C., Fassnacht, C. D., \& Squires, G. K. 2009, ApJ, 690, 295

Kocevski, D. D., Lubin, L. M., Lemaux, B. C., Gal, R. R., Fassnacht, C. D., Lin, R., \& Squires, G. K. 2009, ApJ, 700, 901

Kodama, T., Arimoto, N., Barger, A. J., \& Arag'on-Salamanca, A. 1998, A\&A, 334, 99

Lara-López, M. A., Cepa, J., Bongiovanni, A., Castañeda, H., Pérez García, A. M., Fernández Lorenzo, M., Póvic, M., \& Sánchez-Portal, M. 2009, A\&A, 493, L5

Lemaux, B. C., et al. 2009, ApJ, 700, 20 (Lem09)

Lewis, I., et al. 2002, MNRAS, 334, 673

Liu, X., Shapley, A. E., Coil, A. L., Brinchmann, J., \& Ma, C.-P. 2008, ApJ, 678,758

Lubin, L. M., Gal, R. R., Lemaux, B. C., Kocevski, D. D., \& Squires, G. K. 2009, AJ, 137, 4867 (L09)

Lubin, L. M., Oke, J. B., \& Postman, M. 2002, AJ, 124, 1905

McLean, I. S., et al. 1998, Proc. SPIE, 3354, 566

Miller, C. J., Nichol, R. C., Gómez, P. L., Hopkins, A. M., \& Bernardi, M. 2003, ApJ, 597, 142

Montero-Dorta, A. D., et al. 2009, MNRAS, 392, 125

Moustakas, J., \& Kennicutt, R. C., Jr. 2006, ApJS, 164, 81

Moustakas, J., Kennicutt, R. C., Jr., \& Tremonti, C. A. 2006, ApJ, 642, 775

Mullis, C. R. 2001, PhD thesis, Univ. of Hawaii

Nagao, T., Maiolino, R., \& Marconi, A. 2006, A\&A, 459, 85

Oemler, A., Dressler, A., Kelson, D., Rigby, J., Poggianti, B. M., Fritz, J., Morrison, G., \& Smail, I. 2009, ApJ, 693, 152

Oke, J. B., \& Gunn, J. E. 1983, ApJ, 266, 713

Oke, J. B., Postman, M., \& Lubin, L. M. 1998, AJ, 116, 549

Pérez-Montero, E., et al. 2009, A\&A, 495, 73

Pimbblet, K. A., Smail, I., Edge, A. C., O'Hely, E., Couch, W. J., \& Zabludoff, A. I. 2006, MNRAS, 366, 645

Poggianti, B. M., Smail, I., Dressler, A., Couch, W. J., Barger, A. J., Butcher, H., Ellis, R. S., \& Oemler, A. J. 1999, ApJ, 518, 576

Poggianti, B. M., et al. 2006, ApJ, 642, 188

Quintero, A. D., et al. 2004, ApJ, 602, 190

Rudnick, G., et al. 2006, ApJ, 650, 624

Shields, J. C. 1992, ApJ, 399, L27

Silverman, J. D., et al. 2009, ApJ, 696, 396

Simcoe, R. A., Metzger, M. R., Small, T. A., \& Araya, G. 2000, BAAS, 32, 758

Skrutskie, M. F., et al. 2006, AJ, 131, 1163

Stasińska, G., Cid Fernandes, R., Mateus, A., Sodré, L., \& Asari, N. V. 2006, MNRAS, 371, 972

Stott, J. P., Pimbblet, K. A., Edge, A. C., Smith, G. P., \& Wardlow, J. L. 2009, MNRAS, 394, 2098

Taniguchi, Y., Shioya, Y., \& Murayama, T. 2000, AJ, 120, 1265

Terlevich, R., \& Melnick, J. 1985, MNRAS, 213, 841

Tody, D. 1993, in ASP Conf. Ser. 52, Astronomical Data Analysis Software and Systems II, ed. R. J. Hanisch, R. J. V. Brissenden, \& J. Barnes (San Francisco, CA: ASP), 173 
Tran, K.-V. H., Franx, M., Illingworth, G., Kelson, D. D., \& van Dokkum, P. 2003, ApJ, 599, 865

Tremonti, C. A., et al. 2004, ApJ, 613, 898

Trümper, J. 1982, Adv. Space Res., 2, 241

van Dokkum, P. G., Franx, M., Fabricant, D., Illingworth, G. D., \& Kelson, D. D. 2000, ApJ, 541, 95

Veilleux, S., Kim, D.-C., Sanders, D. B., Mazzarella, J. M., \& Soifer, B. T. 1995, ApJS, 98, 171

Veilleux, S., \& Osterbrock, D. E. 1987, ApJS, 63, 295

Vergani, D., et al. 2008, A\&A, 487, 89
Wang, B., \& Heckman, T. M. 1996, ApJ, 457, 645

Weiner, B. J., et al. 2007, ApJ, 660, L39

Wild, V., Walcher, C. J., Johansson, P. H., Tresse, L., Charlot, S., Pollo, A., Le Fèvre, O., \& de Ravel, L. 2009, MNRAS, 395, 144

Yan, R., Newman, J. A., Faber, S. M., Konidaris, N., Koo, D., \& Davis, M. 2006, ApJ, 648, 281 (Y06)

Yan, R., et al. 2009, MNRAS, 398, 735

Zabludoff, A. I., Zaritsky, D., Lin, H., Tucker, D., Hashimoto, Y. Shectman, S. A., Oemler, A., \& Kirshner, R. P. 1996, ApJ, 466, 104 